Cambridge Archaeological Journal

http://journals.cambridge.org/CAJ

Additional services for Cambridge Archaeological Journal:

CAMBRIDGE

Email alerts: $\underline{\text { Click here }}$

Subscriptions: $\underline{\text { Click here }}$

Commercial reprints: $\underline{\text { Click here }}$

Terms of use : $\underline{\text { Click here }}$

\title{
A New Look at the Berekhat Ram Figurine: Implications for the Origins of Symbolism
}

Francesco d'Errico and April Nowell

Cambridge Archaeological Journal / Volume 10 / Issue 01 / April 2000, pp 123 - 167

DOI: 10.1017/S0959774300000056, Published online: 27 June 2001

Link to this article: http://journals.cambridge.org/abstract_S0959774300000056

How to cite this article:

Francesco d'Errico and April Nowell (2000). A New Look at the Berekhat Ram Figurine: Implications for the Origins of Symbolism. Cambridge Archaeological Journal, 10, pp 123-167 doi:10.1017/S0959774300000056

Request Permissions : $\underline{\text { Click here }}$ 


\title{
A New Look at the Berekhat Ram Figurine: Implications for the Origins of Symbolism
}

\author{
Francesco d'Errico \& April Nowell
}

\begin{abstract}
This article addresses the nature of the evidence for symbolling behaviour among hominids living in the Near East during the Middle and Upper Pleistocene. Traditionally, Palaeolithic art and symbolling have been synonymous with the Upper Palaeolithic of Europe. The Berekhat Ram figurine, a piece of volcanic material from a Lower Palaeolithic site in Israel, described as purposely modified to produce human features, challenges the view of a late emergence of symbolic behaviour. The anthropogenic nature of these modifications, however, is controversial. We address this problem through an examination of volcanic material from the Berekhat Ram site and from other sources, and by experimentally reproducing the modifications observed on the figurine. We also analyze this material and the figurine itself through optical and SEM microscopy. Our conclusion is that this object was purposely modified by hominids.
\end{abstract}

In Palaeolithic archaeology much debate surrounds the origin and development of hominid symbolic behaviour. For some researchers the emergence of this capacity is associated with the transition from the Middle to Upper Palaeolithic in Europe (e.g. Chase \& Dibble 1987; Davidson \& Noble 1989; 1993; Noble \& Davidson 1996; Mellars 1989; 1991; 1996; White 1989; 1992; 1993; Stringer \& Gamble 1993; Byers 1994; Klein 1996; Mithen 1996a). According to these authors, anatomically modern humans arriving in Europe developed the use of body ornaments and a complex repertoire of abstract and depictional art. These behaviours imply the use of symbols for the first time. An alternative view is that the emergence of symbolling is a gradual process whose roots can be traced back to the Lower and Middle Palaeolithic (Marshack 1976; 1988; 1991b; 1995a; Bednarik 1992; 1994; 1995; 1997; Bahn 1996; Duff et al. 1992; Simek 1992; Hayden 1993; Wolpoff \& Caspari 1996).

Taking a discontinuist or gradualist approach depends greatly on one's evaluation of the evidence for symbolling prior to the Upper Palaeolithic. This evidence includes utilized pieces of colorant, 'curated' fossils, crystals and shells, putative engraved and perforated bones and stones, and possible musical instruments (Turk 1997). For some proponents of the discontinuity model, many of the objects often interpreted as symbolic are in actuality the result of natural processes, e.g. carnivore activity, chemical alterations, root marking, etc. This view has been supported in some cases by comparing bone objects possessing putative anthropogenic engravings or perforations from Lower and Middle Palaeolithic sites with bone fragments altered by known causes (Chase 1990; d'Errico 1991; d'Errico \& Villa 1997; d'Errico et al. 1998b). In addition, discontinuity proponents argue that, even if humanly made, some objects described as 'symbolic' by the gradualists can have a purely utilitarian function (Chase \& Dibble 1992). The paucity and uniqueness of these objects is taken as further proof of the absence of a symbolic tradition which would require a spatial and temporal continuity in the production and use of symbols (Chase \& Dibble 1992; Noble \& Davidson 1996, 210).

A further critique of the gradualist viewpoint is that 'art' objects are often not considered in the context of the assemblage as a whole. A contextual approach is needed to 'eliminate the possibility that the piece, far from being an idiosyncratic example of 


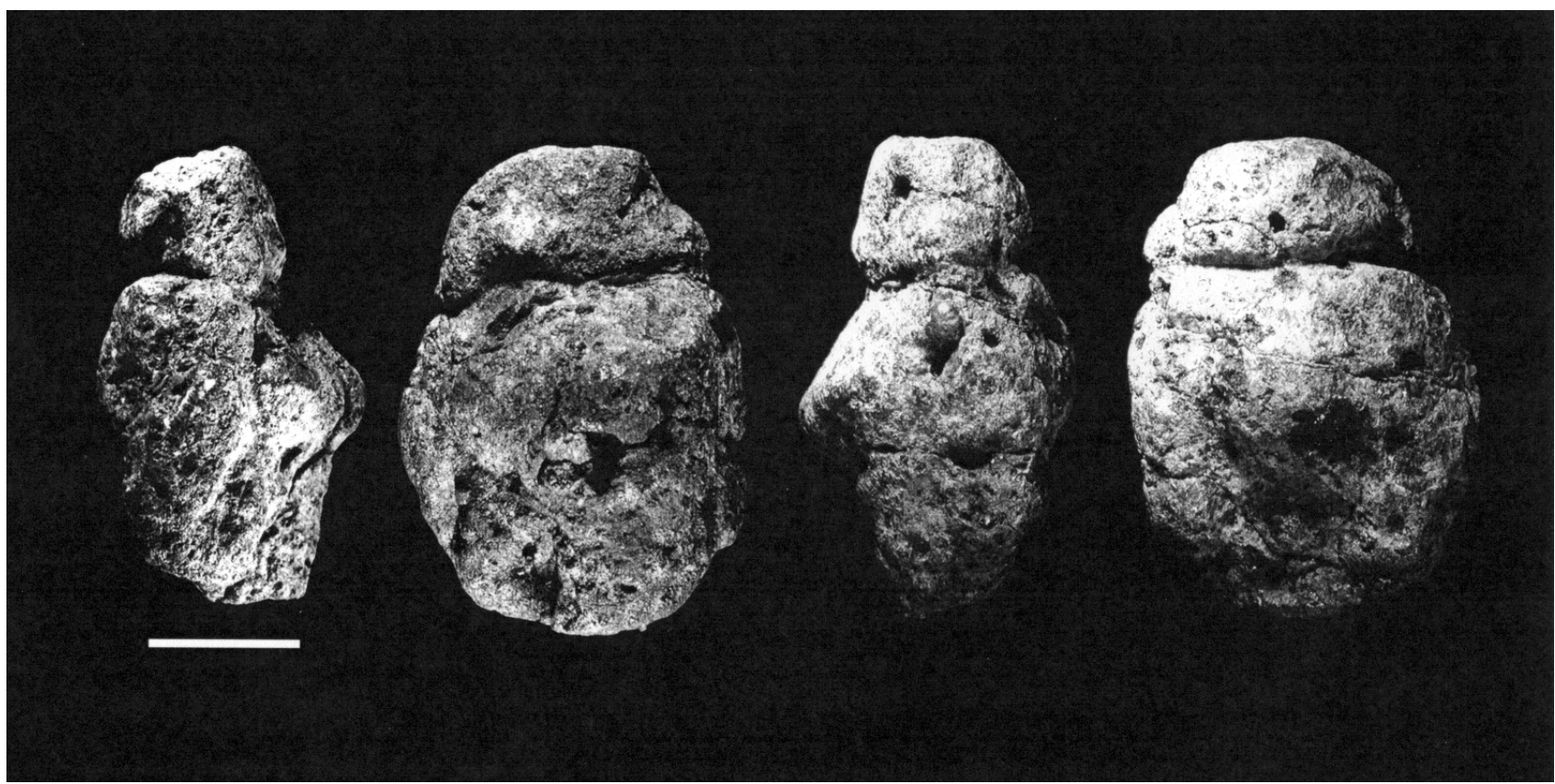

Figure 1. Berekhat Ram figurine. Four views $($ scale $=1 \mathrm{~cm})$.

a rare behaviour, is only an individual within a population of similar but less striking [natural] phenomena' (d'Errico \& Villa 1997, 28). Finally, the most common critique made by advocates of discontinuity is that, owing to their small size, body ornaments and engraved objects may be found in older layers as a consequence of post-depositional disturbances. It has been shown experimentally and confirmed through refitting studies that the vertical displacement of small objects is likely in sediments that were unconsolidated at the time of the occupation (GiffordGonzalez et al. 1985; Villa \& Courtin 1983).

Gradualists counter that the rarity in these early periods of objects documenting the use of symbols over a long period of time is the consequence of taphonomic processes. These processes deprive the archaeological record of objects made of perishable materials and dramatically reduce the number of those produced on more durable ones (Bednarik 1992; 1995). Gradualists also believe that discontinuists employ stricter criteria when evaluating objects from the Lower and Middle Palaeolithic than those from later periods (Bednarik 1995). Furthermore, they assert that discontinuists use the Upper Palaeolithic as a measure for establishing the kinds of artefacts that should be discovered in earlier periods to demonstrate the use of symbols. Finally, these researchers argue that the discontinuists' view of the transition from Middle to Upper Palaeolithic is coloured by an overemphasis on the West European record where the relatively sudden appearance of cave art and body ornament gives the impression of a rupture between these two periods that is not apparent in other regions.

In recent years, claims for symbolic objects from outside Western Europe dating to the Lower and Middle Palaeolithic have added fuel to this debate. Recently, in Bulgaria, a slab engraved with parallel lines was found associated with a transitional industry between the last Mousterian and the first Aurignacian levels (Crémades et al. 1995). The level from which the object derives has been attributed to 50,000 вр. According to one of the scholars in charge of the excavation, however (Guadelli pers. comm.), this slab could be younger. The only conventional radiocarbon date available for the level in question indicates a minimum age of 38,700 вр (Gd-4687). The older age has been suggested on the basis of a stratigraphic correlation with levels dated by TL but these levels were excavated in an area of the cave that is $30 \mathrm{~m}$ away from the pit where the slab was found. At Prolom II, a Middle Palaeolithic cave site in the Eastern Crimea, engraved and perforated bone fragments have been reported (Stepanchuk 1993). In Slovenia, claims (Turk et al. 1995; Turk 1997) have been made for the earliest musical instrument, a Neanderthal bone flute, but several scholars suggest that this object is simply a heavily carnivore-gnawed 
bone (see Chase \& Nowell 1998; d'Errico et al. 1998b; in press). Engraved ostrich eggshells, clear bone tools, serially notched objects and hundreds of pieces of utilized ochre were found in a Middle Stone Age context at several sites in South Africa (Wendt 1974; Singer \& Wymer 1982; Knight et al. 1995; Henshilwood \& Sealy 1997). In Libya, researchers found fragments of ostrich eggshell beads that are apparently associated with Acheulean tools (Bednarik 1997). Researchers have also reported engraved parietal art in Australia possibly dating to 75,000 вр (Fullagar et al. 1996), a continent where the oldest evidence of body ornament now dates back to more than 30,000 вр (Morse 1993).

The most striking discoveries, however, have come from the Near East, where a carved figurine has been reported from the Lower Palaeolithic site of Berekhat Ram (Goren-Inbar 1986; Marshack 1995b; 1997a,b). In addition, engraved cortexes have been reported from the Middle Palaeolithic sites of Quneitra (Goren-Inbar 1990; Marshack 1995a) and Qafzeh (Hovers et al. 1997). Perforated and ochred Glycymeris shells were found at Qafzeh in association with early modern human burials dating to 100,000 BP. Finally, the Middle Palaeolithic site of Skhul has also yielded similar shells (Bar-Yosef 1992; Vandermeersh pers. comm.).

While not exhaustive, this list illustrates the number and variety of new objects coming from outside of Western Europe that predate the Upper Palaeolithic. The significance of these objects for the debate surrounding the origins of symbolism remains unclear, however, as researchers have not created actualistic and comparative models with which to evaluate both the anthropogenic and symbolic nature of these findings. In this article we focus on one of the most puzzling objects discovered in the Near East in the last decade - the Berekhat Ram figurine (Fig. 1). This object, a putative engraved fragment of volcanic material, is important because it is possibly the earliest example of representational art (GorenInbar 1986; Schepartz 1993; Marshack 1997a,b; Bahn 1996; Bahn \& Vertut 1997).

\section{Archaeological context and initial research}

The Berekhat Ram site, in the Golan Heights region of Israel, excavated by Goren-Inbar in 1981 and 1982, is on the edge of the crater of an extinct volcano which is now partially filled by a natural lake (Mor 1981). The figurine, in association with Acheulean tools (Fig. 2), derives from an archaeological horizon, $25 \mathrm{~cm}$ thick (Fig. 3), lying within a $1 \mathrm{~m}$ colluvial / alluvial unit which is sandwiched between two basalt flows, known as the Lower and Upper Kramim basalts (Goren-Inbar 1985; Goren-Inbar et al. 1986). Using $\mathrm{Ar}^{40} / \mathrm{Ar}^{39}$, these basaltic layers have been dated to 470,000 BP and 233,000 BP respectively (see Feraud et al. 1983 for a discussion of dating results; see also Goldberg 1987 for a discussion of the geology of the archaeological layers). The archaeological layer where the figurine was found is estimated to date to approximately $250,000-280,000 \mathrm{BP}$.

The lithic industry at Berekhat Ram, described as Acheulean, is somewhat unusual in that Upper Palaeolithic types (burins, borers, endscrapers) comprise 20.6 per cent of the assemblage. It is also rich in Levallois flakes, sidescrapers, notches and denticulates. Levallois cores represent the most common core type. The eight bifaces found at the site are all of a small size (less than $11 \mathrm{~cm}$ ). With the exception of one biface manufactured from basalt, all artefacts are made from local flint (Goren-Inbar 1985).

According to Goren-Inbar (1986), hominids selected a scoria pebble that bore natural features resembling a female figure. It is $35 \mathrm{~mm}$ in length, 25 $\mathrm{mm}$ in width and $21 \mathrm{~mm}$ in thickness. The tuff was then modified to enhance these natural features by carving one deep groove around the fragment at the upper third of the piece to delineate a neck and head. Two shallow, curved grooves were engraved to create the arms of the figurine. The anthropogenic origins of these grooves have been called into question by Pelcin (1994; see also Davidson 1990; Noble \& Davidson 1996, 75). Pelcin points out that cracks or fissures on the surface of scoria, produced during the ejection and cooling of this material, can be mistaken for grooves produced by a stone tool. In particular, he suggests that the groove delineating the head could have been produced in the air or upon impact. According to Pelcin, a comparative approach involving microscopic analysis is needed to demonstrate that the grooves described on the figurine are different from those that may be present on the many tuff lapilli found within the archaeological layer from which the figurine derives.

Goren-Inbar \& Peltz (1995) replied to this article by emphasizing that the figurine was not made of scoria, as previously published (Goren-Inbar 1986, 7), but was in fact made of a basaltic lapillus tuff incorporating scoria clasts, implying that the type of natural grooves described by Pelcin, which are characteristic of scoria, are not present on this material. They do not, however, address Pelcin's call for a comparative approach. As a consequence of this, Goren-Inbar \& Peltz do not demonstrate that natural 

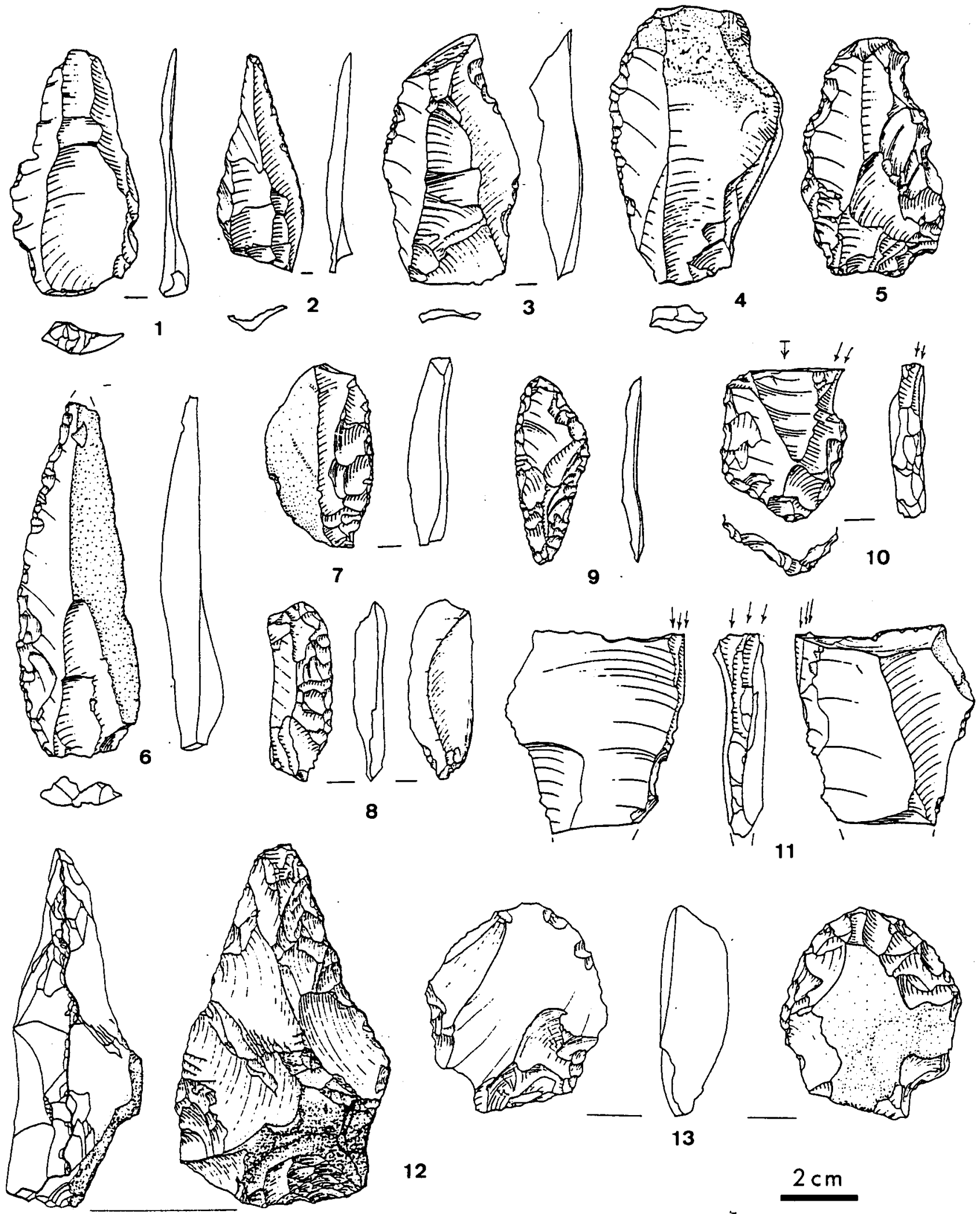

Figure 2. Stone tools found at Berekhat Ram: 1-3) flakes; 4, 6-8) side scrapers; 5) denticulate; 9) double-side scraper; 10-11) burins; 12) hand-axe; 13) end-scraper. (After Goren-Inbar 1985.) 
grooves, possibly mimicking anthropogenic engravings, are absent on basaltic lapilli tuff.

Noble \& Davidson support the position that hominids were not involved in the creation of the figurine. According to these researchers, there is no evidence that the 'Berekhat Ram object was formed by human agency rather than by natural forces . . . hence we cannot say that it was a thing made to resemble anything' $(1996,75)$. They assert that if the object was not modified by hominids, archaeologists cannot know whether it was seen as a figurine or anything else in the Acheulean. Noble \& Davidson further believe that this object is considered a figurine simply because in the minds of the archaeologists working at the site it resembled Venus figurines from the European Upper Palaeolithic. In other words, the archaeologists must have perceived certain features of the object as reminiscent of a style of art that appears some 200,000 years later in Europe. The iconic relationship established by archaeologists, however, should not be considered as self-evident.

Marshack (1997a,b) has recently published a new detailed description of the figurine. In agreement with Goren-Inbar (1986), Marshack asserts that the object's natural features, reminiscent of the female anatomy, were enhanced by hominids to produce a female figure. By recognizing these different 'body parts', Marshack is able to identify the front and rear of the figurine, and several other anatomical details. Teasing apart the natural features of the figurine from those considered by Marshack as intentionally modified is a difficult task as he blends them together in his description. According to Marshack, the intentional modifications, as we understand them, include a circular groove delineating the neck of the figure; another groove on its right side defining a thick arm bent at the elbow; scraping and flattening of the right shoulder; a flat plane on the chest, obtained by scraping, which separates it from the right arm, which is also delineated by an anthropogenic groove; artificial smoothing of the lower plane of the neck groove; and careful bevelling of the right protuberance of the head.

Marshack's identification of the anthropogenic markings on the figurine is problematic for several reasons. First, he has not carried out a comparative study of volcanic material from the Berekhat Ram site or from other volcanic sites to know whether or not grooves appear naturally on this type of material and to acquire a direct knowledge of the morphological variability of volcanic tuff. Neither has he

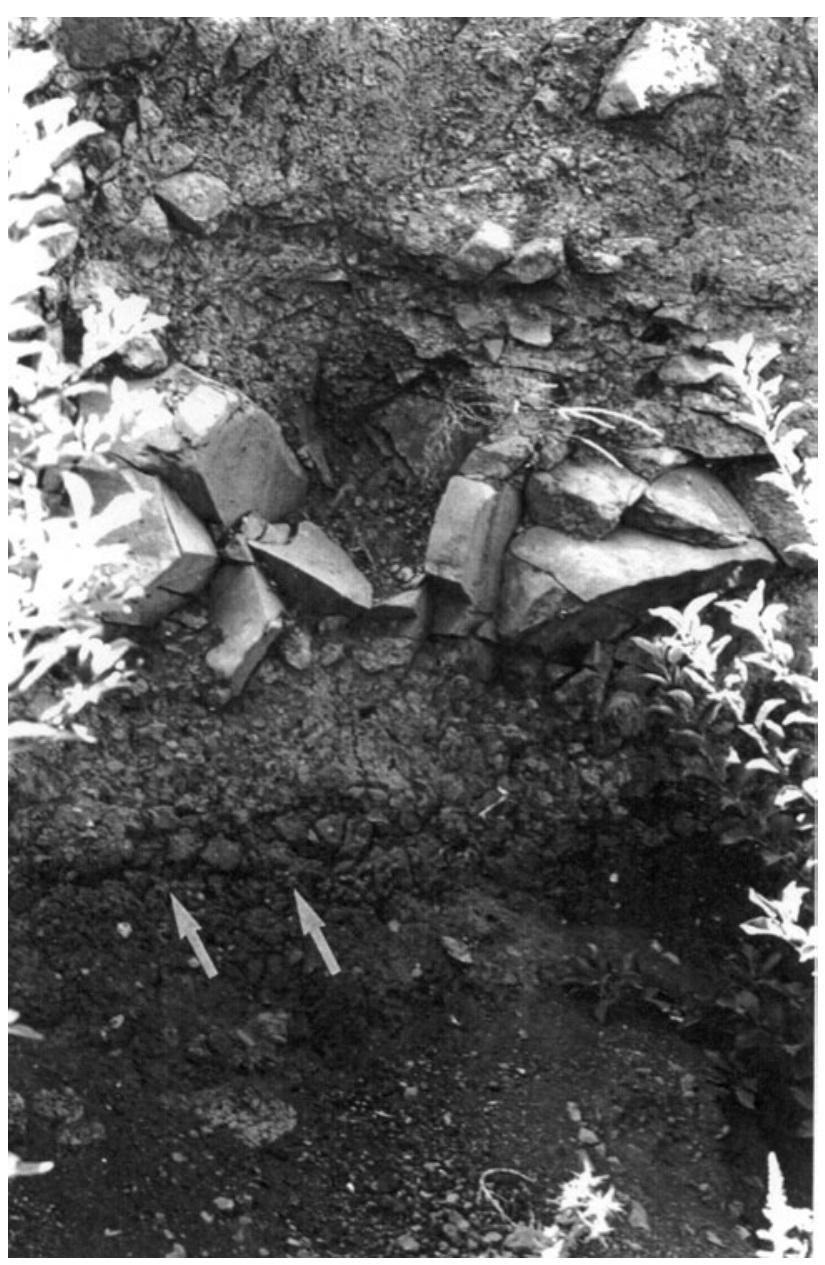

Figure 3. Section of site exposed showing the Upper Kramim basalt and the colluvial/alluvial unit containing the archaeological layer (arrows).

conducted any experiments to ascertain what traces stone tools produce on this raw material. His conclusions concerning the anthropogenic nature of the figurine are based, therefore, on three assumptions: (1) there are no natural grooves on this type of raw material; (2) if any natural grooves did exist they would not resemble those on the figurine; (3) one can infer the techniques and the type of tool used to produce intentional grooves and other types of anthropogenic modification (abrasion, scraping) on this type of very unusual material through simple observation of the object's surface morphology. In other words, his diagnosis of the hominid-produced modifications are based on what he believes these markings would look like and not on criteria he has established through experimentation and comparative microscopic analysis.

Second, even if the figurine's grooves are in- 


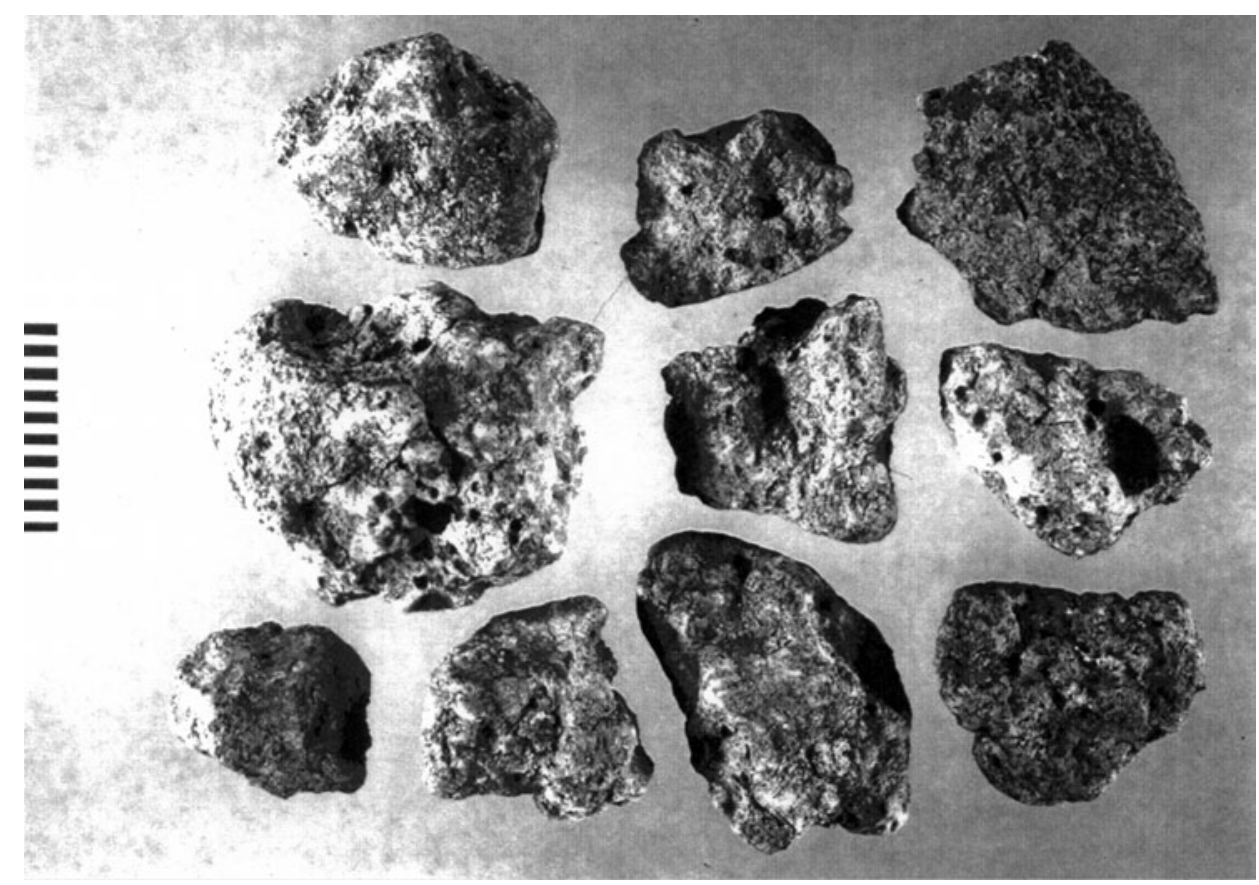

Figure 4. Lapilli tuff fragments from the section exposed at the Berekhat Ram site.

\section{Methodology}

The archaeological excavations at Berekhat Ram and later digging for agricultural purposes have led to the creation of an artificial terrace on the western side of the Berekhat crater lake, exposing a $150 \mathrm{~m}$ section of the Upper Kramim basalt and of the colluvial/alluvial unit containing the archaeological layer (Fig. 3). The position of this layer was clearly identified in the field from the in situ presence of several stone artefacts with the characteristic reddishbrown patina typical of the assemblage found in the 1980-81 excavation (GorenInbar 1985).

A range of volcanic deed anthropogenic, it is still unclear why the object should be interpreted as a human figure and oriented in the manner suggested by Marshack. On crude or somehow schematic figures, such identification depends on the diagnostic criteria one accepts as valid to recognize carved or depicted human representations (Rosenfeld 1977; Duhard 1993a,b; see also discussion by Noble \& Davidson 1996, 75-7). Marshack, however, does not make the criteria he employs explicit. As a consequence, he maintains that the figurine is indeed such without discussing possible alternative interpretations.

A related problem concerns the symbolic value of the figurine. Grooves can be produced for a variety of functional reasons, and, even if we accept that the object is representational, what is its significance? Does its carving at an Acheulean site imply the use of symbols by its makers? The difficulties inherent in Goren-Inbar's (1986) and Marshack's (1997a) analyses and the criticisms of Pelcin (1994) and Noble \& Davidson (1996) have prompted us to undertake a re-examination of the Berekhat Ram figurine. Our study differs from previous work on the subject in that we have subjected the figurine, unmodified raw material from this site and from other volcanic sites in Israel, and experimentally modified raw material to both optical and SEM microscopy in order to develop criteria by which to evaluate the anthropogenic origin of the markings. raw material was collected from the colluvial/alluvial unit exposed by this section. This sample includes large fragments from the Upper Kramim basalt, basalt pebbles, reddish basaltic lapilli tuffs, and fragments of yellowish-red, hard, coarse, crumbly clay, making a total of 100 pieces. Our examination in the field of several hundred volcanic clasts extracted from the archaeological layers guarantees that the collected sample is representative of the different volcanic materials present at the site. Some of the lapilli tuffs (Fig. 4) show a resemblance in colour, size, texture and composition to the figurine. These pieces were examined for natural features which might mimic humanly made grooved, scraped and abraded areas, using a Wild M3C reflected and transmitted light microscope equipped with a photo system.

Local flint, from the same sources as those exploited by hominids at Berekhat Ram, was knapped to produce replicas of flakes and tools found at the site (burins, sidescrapers, and points). A selected sample of the lapilli tuffs was experimentally grooved in a back-and-forth motion with the cutting edge of both blanks and retouched flakes or with a burin or a point tip. Retouched and unretouched flakes were used to scrape the surface of the fragments. Some were also abraded against a large basalt flake. Experimentally modified surfaces were examined under the reflected light microscope before and after being washed with water and a soft brush. 
A large sample of volcanic scoria from northern Israel (approximately 3000 pieces) was also examined. Thirty pieces similar in size to the figurine and bearing natural grooves were selected and examined under the optical microscope and by SEM. The collection of scoria clasts and lapilli tuff fragments of different provenance kept at the Department of Geology, University of Bordeaux, was also examined for comparison. Some of these fragments show grooves that are similar to those illustrated by Pelcin (1994, figs. 1-2).

The Berekhat Ram figurine, on exhibit at the Israel Museum in Jerusalem, was examined with the same optical microscope in order to identify and document possible artificial surface modifications. Several sections of the figurine's neck groove were obtained by positioning the object so that the groove profile was in the lens axis and then enhancing the profile by observing it in both transmitted and reflected light. This same procedure was applied to the grooves produced experimentally. Archaeological sections were recorded by photography. Slides so obtained were digitized and analyzed with the same method used for experimental grooves. The experimental sections were recorded using a CCD KP-M1E/K Hitachi camera mounted on the microscope and connected to a desktop computer Power Macintosh ${ }^{\mathrm{TM}}$ equipped with a Kingfisher (Graphics Unlimited, UK) frame grabber card. Through a series of image enhancement steps, using NIH Image software, the curved line of the profile can be transformed into numerical values (d'Errico 1998). The plot so produced may be used for visual comparison with plots of other grooves. Quantification of morphological differences between profiles can be obtained by measuring angles and computing polynomial equations or conventional Fourier functions (Gero \& Mazzullo 1984; Lestrel 1989).

The figurine was also examined with a JSM5410LV Jeol low pressure SEM at the Faculty of Agriculture, Hebrew University, Rehovot. Unmodified and experimentally modified tuff fragments from the site, and the naturally grooved scoria sample were examined with a JXA 8600 Jeol SEM at the INASMET Laboratory, San Sebastian, Spain and with a LEO 435VP SEM at the laboratory of the French LEO representative (Rueil-Malmaison).

\section{Results}

Lapilli tuffs from the site

Our first objective was to document the ways in which natural lapilli tuff and scoria might differ from or resemble the figurine. Basaltic lapilli tuffs were collected at the site in the form of whole slightly rounded clasts or as fragments whose fractured surfaces allow observation of the internal structure of the volcanic rock. A few of the specimens preserve areas where the original surface has not been removed by erosion. These consist of dark brown to black highly smoothed surfaces covered by softened superficial microcraters up to $2-3 \mathrm{~mm}$ in diameter, and encrusted with compacted deposits of iron oxide (Fig. 5). The flaking off of this thin layer, common in the more exposed areas, creates rough surfaces with microcraters revealing a fine-grain reddish matrix (Figs. 6 \& 7) and, in most cases, spherical or elliptical vesicles with black walls. Vesicles are commonly visible along the fractured surface of the broken clasts. They may be empty, or be filled with a black or mix of a red-black matrix (Fig. 8). Their maximum diameter generally ranges between 1 and $4 \mathrm{~mm}$ but can reach $9 \mathrm{~mm}$. In a few cases vesicles contain black, glassy granules. Thin microcracks, interpreted by Goren-Inbar and Peltz as the result of the high induration of the rock, as well as different types of inclusions, were also observed on a number of fragments.

While all the above features are equally present on the Berekhat Ram figurine (see below), none of the tuff fragments observed in the field or collected for further examination in the laboratory exhibited grooves of any size or shape which could be confused with those present on the archaeological specimen. Slight differences in colour and in the appearance of the inclusions exist between the sample collected at the site and the figurine. The only notable difference, however, seems to be the presence on the figurine of a small pebble situated in the area that Marshack identified as the shoulder of the figurine (Fig. 1). None of the tuff casts collected at the site include such pebbles. This could indicate (Goren-Inbar pers. comm.) that the figurine was carved on a piece of tuff collected away from the site rather than on a locally available tuff fragment.

\section{Scoria}

All the scoria fragments from our comparative collection are much harder than the tuff fragments collected at the site. They also differ in texture and, unlike the latter, show no inclusions. Some bear grooves that are similar in size and depth to those observed on the figurine (Fig. 9a). These grooves, however, can hardly be confused with those produced experimentally on tuff fragments collected at the site (Figs. $10 \& 11$ ) or with those present on the 

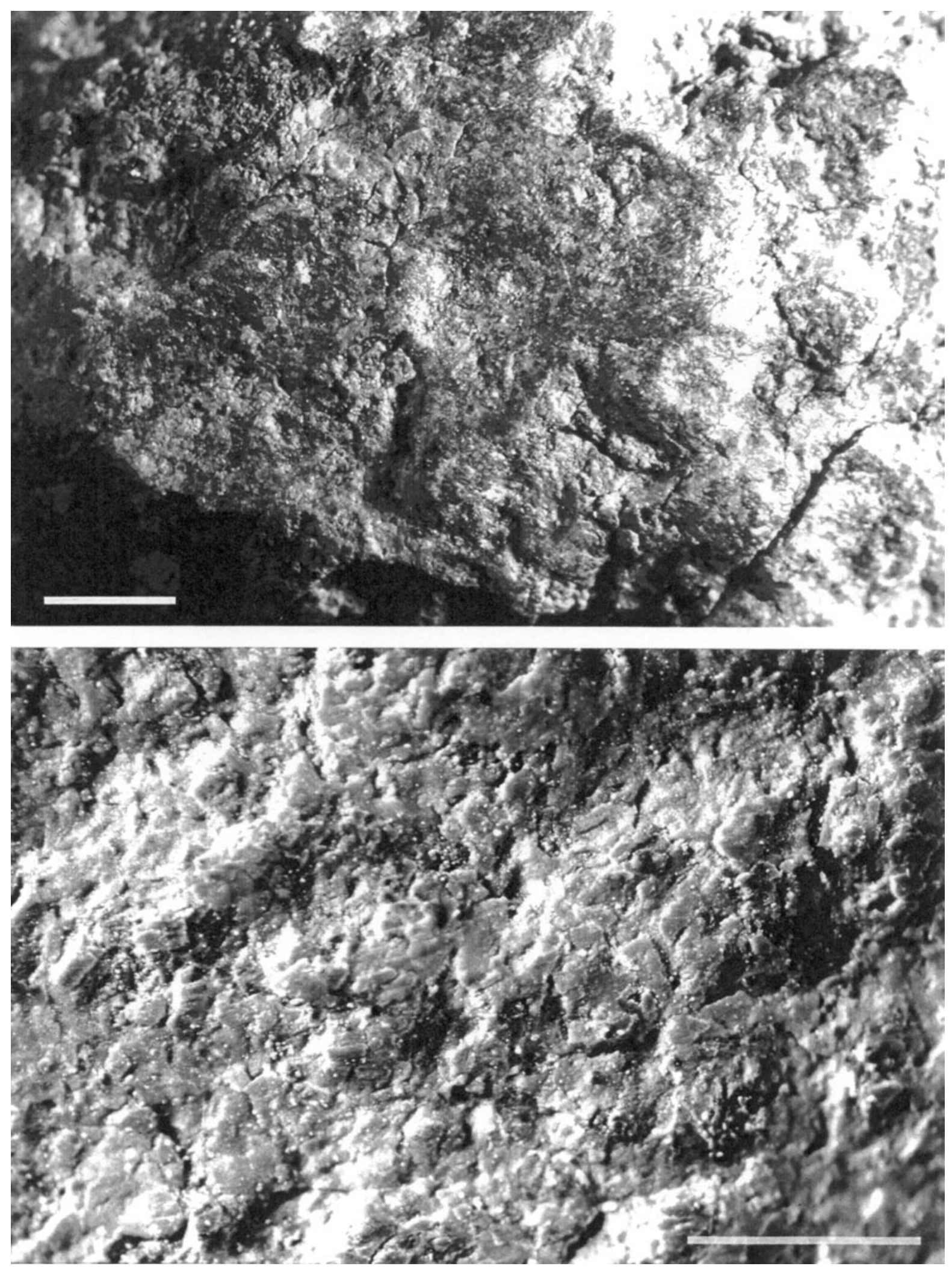

Figure 5. Tuff clasts from the Berekhat Ram Acheulian layer showing a well-preserved surface morphology (scale =1 mm). 


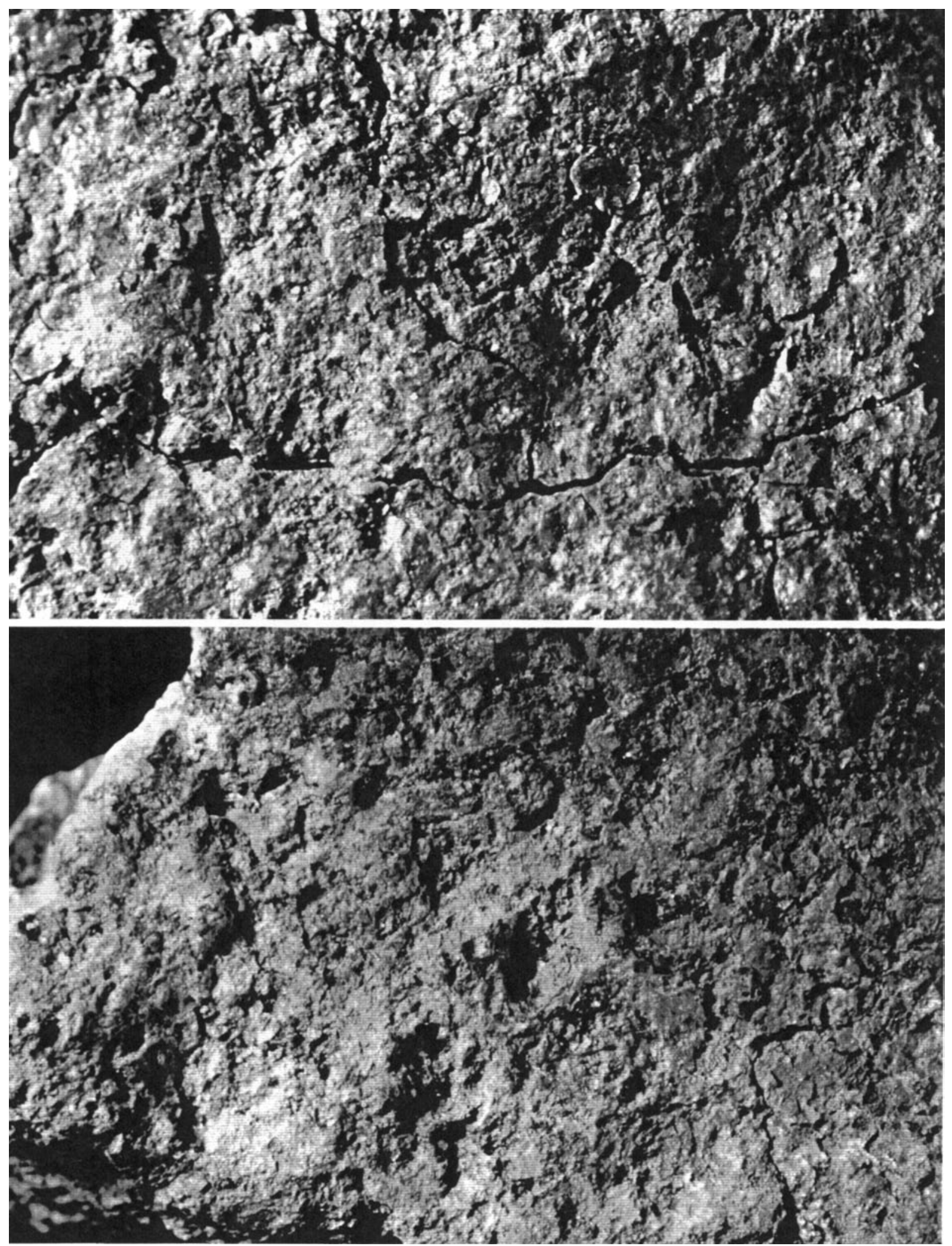

Figure 6. Two examples of tuff surfaces at different stages of erosion. Vestigial compacted areas are still visible in limited places (scale $=1 \mathrm{~mm}$ ). 


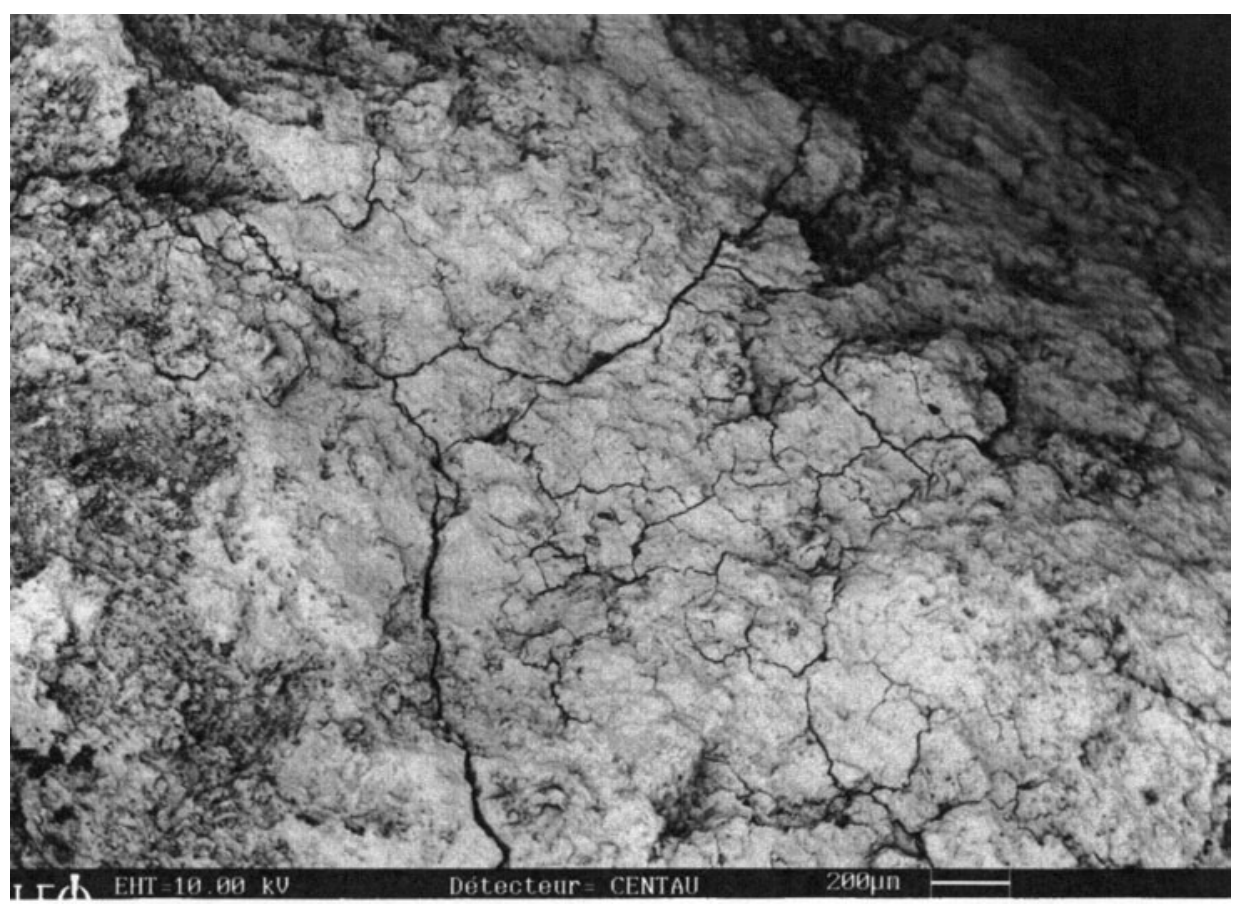

Figure 7. SEM photograph of the natural surface of a well-preserved tuff clast showing the progressive cracking and spalling of the thin, compacted original surface, revealing the rough texture of the inner structure of the clast.

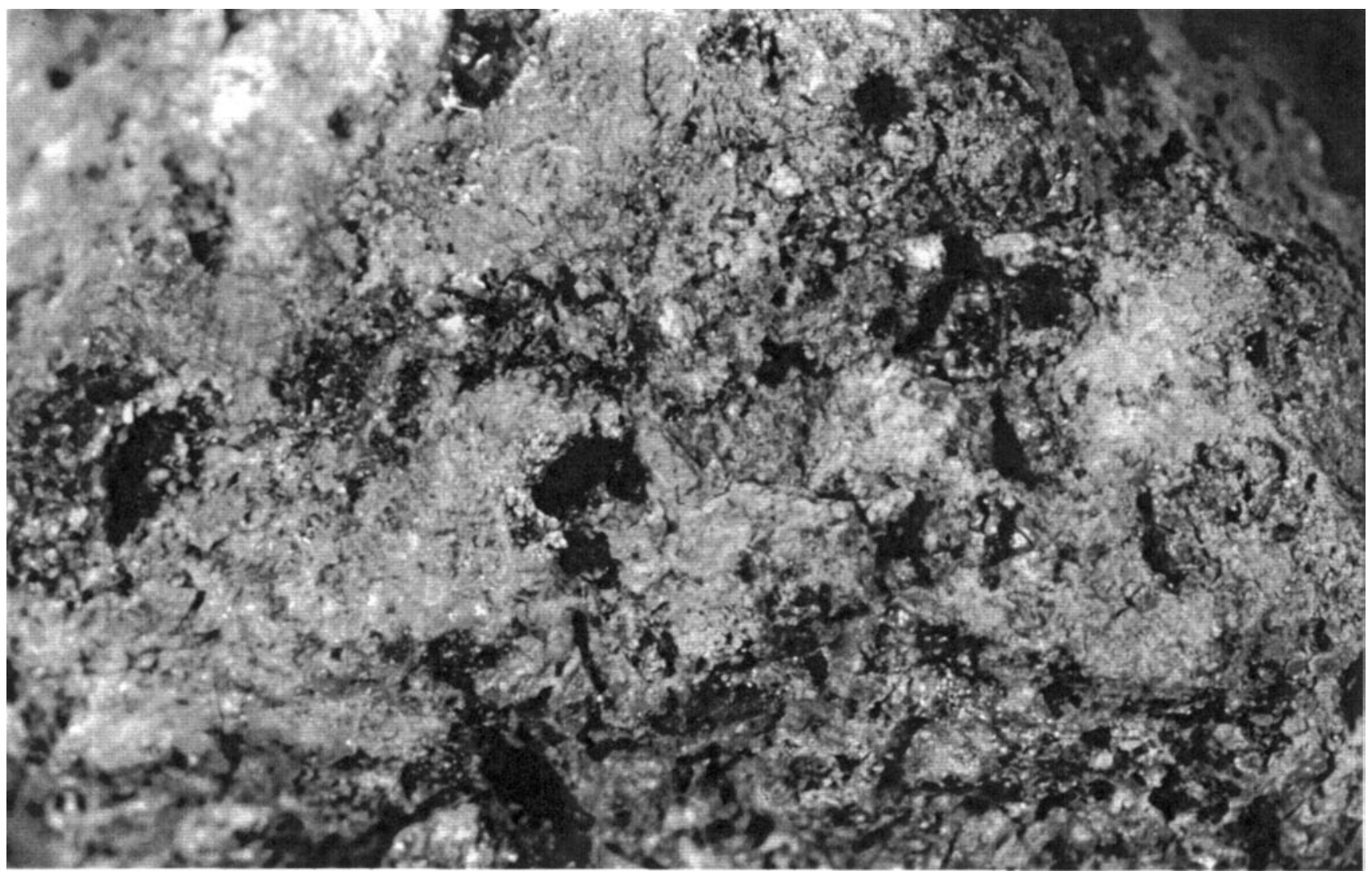

Figure 8. Black vesicles inside a tuff clast (scale $=1 \mathrm{~mm}$ ). 

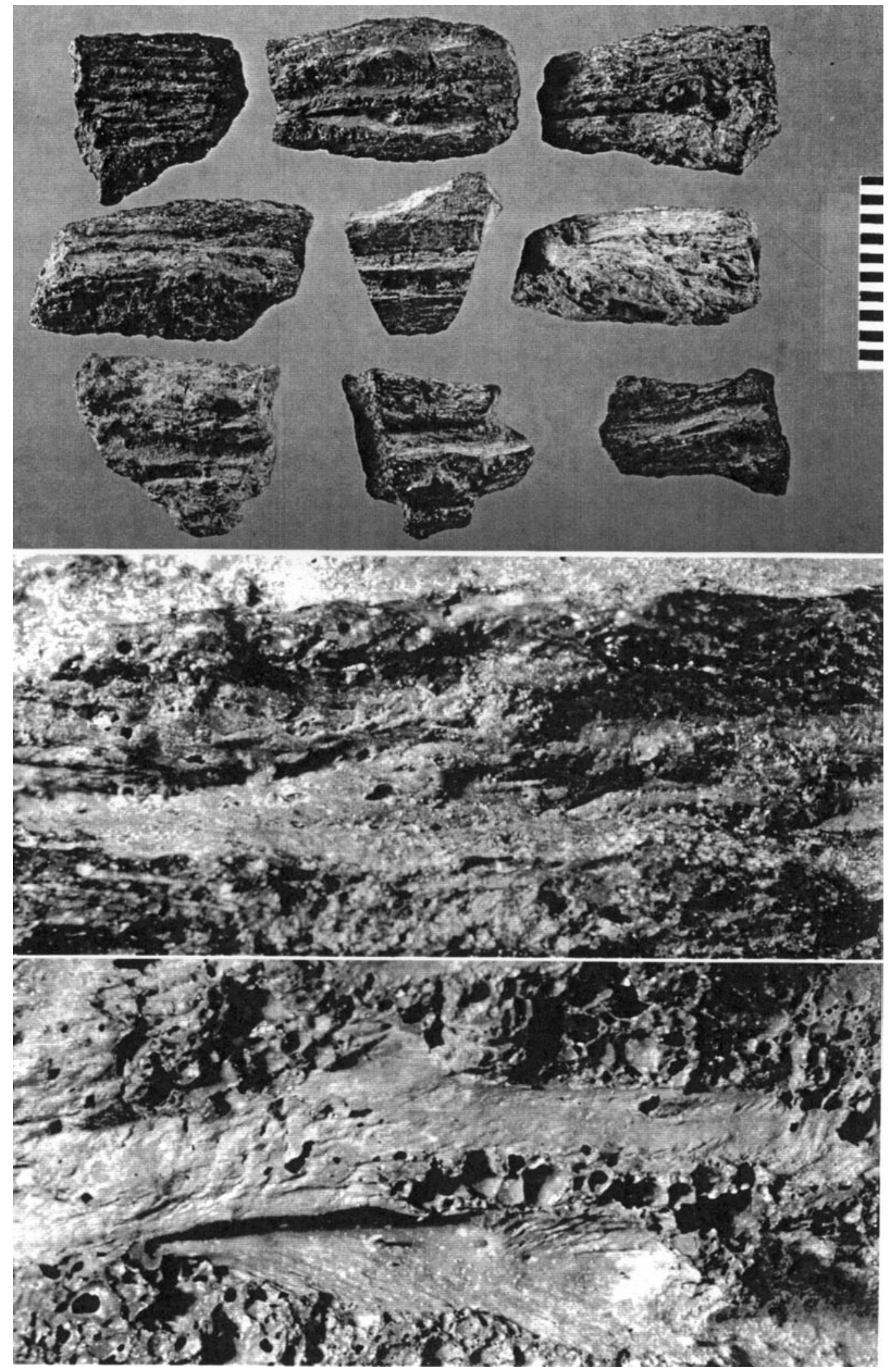

Figure 9. Top: Scoria fragments similar in size to the Berekhat Ram figurine, with unique or multiple parallel natural grooves; below: grooves on scoria showing oblique microfolding and elongated gaps (scale $=1 \mathrm{~mm}$ ). 


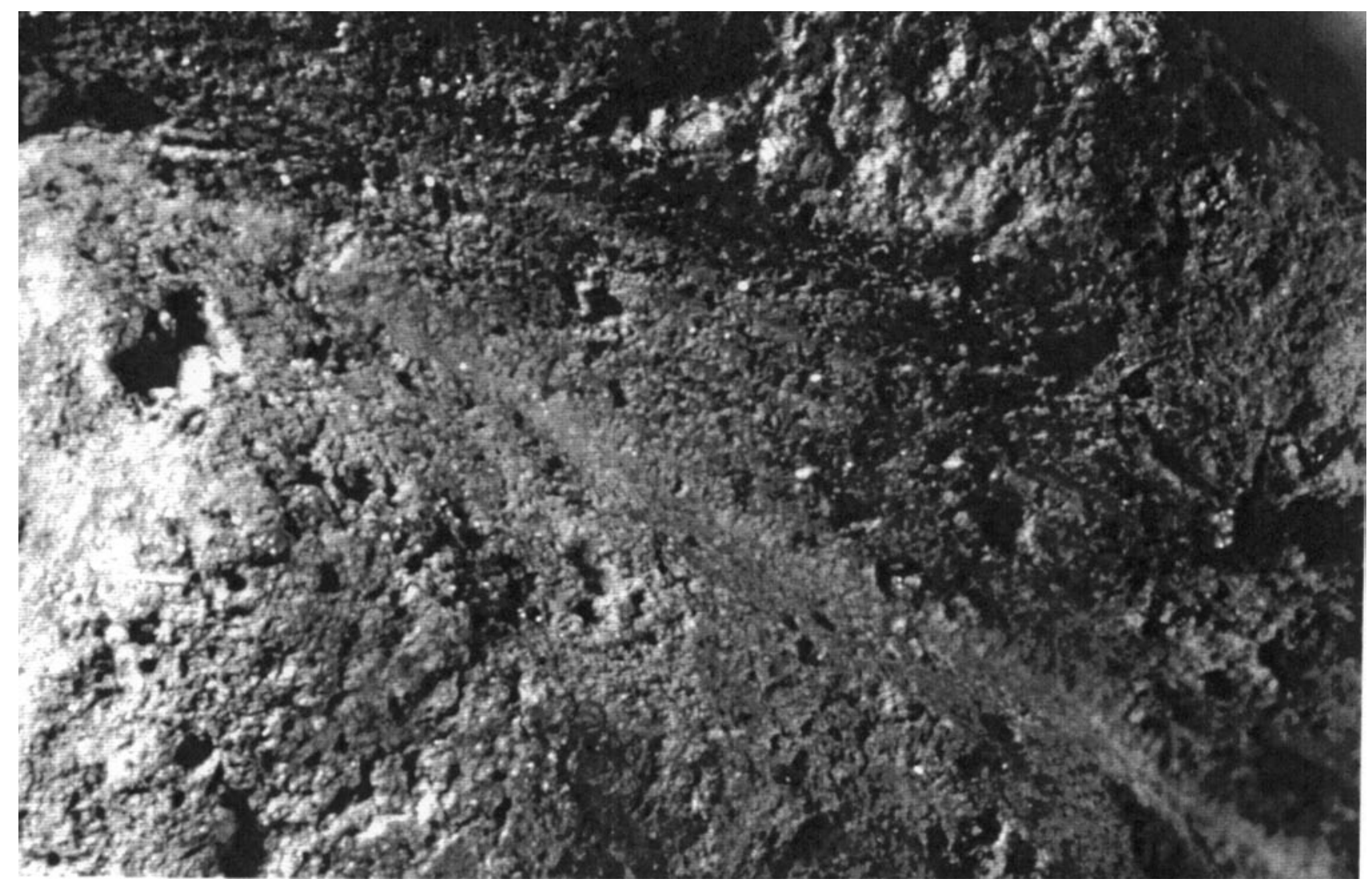

Figure 10. Experimental groove made by a retouched blank on a tuff fragment (scale = $1 \mathrm{~mm}$ ).

archaeological specimen (see below Fig. 21 of neck groove). Scoria generally show multiple parallel or subparallel grooves on one or, less commonly, on both faces of the fragment. These grooves are in fact foldings resulting from the drawing out of the material when it was in a plastic state. Fragments showing unique grooves are rare and in none of the fragments from our comparative collection have we found grooves encircling the clast's volume. Microscopic analysis of the groove surface (Fig. $9 \mathrm{~b}-\mathrm{c}$ ) shows oblique micro-foldings and elongated gaps demonstrating that the grooves are the results of the rapid hardening of soft material. No striations which could possibly be mistaken with those produced experimentally on tuff fragments were observed on the walls of the grooves of the scoria fragments.

\section{Experimental engraving}

Microscopic analysis

At microscopic scale, the surface of grooves carved by flint tools is different from that observed on natural, well-preserved tuff clasts and on altered frag- ments (Fig. 10). The repeated motion of the tool's cutting edge breaks off pieces of material and grinds these particles against the groove walls and foundation. As a consequence, the groove surfaces are flat, slightly porous, and show a texture more homogeneous than that visible on their natural counterpart (Fig. 11). Vesicles intercepted by the groove appear in the form of concave, highly porous areas filled with large granules. Sections of inclusions pulled by the tool emerge from the groove walls. The foundation of the groove is often covered with a fine powder, resulting from the crushing of the tuff fragments under the pressure exerted by the stone tool in cutting the groove. Shallow, irregular striations are often visible on the groove walls (Fig. 10).

\section{Identification of the engraving tool}

Circular grooves produced on a tuff clast by the toand-fro movement of an unretouched blank can be distinguished from those made with a retouched tool by examining a section of the groove and by measuring the angle formed by its walls. Grooves produced by unretouched blanks generally have 

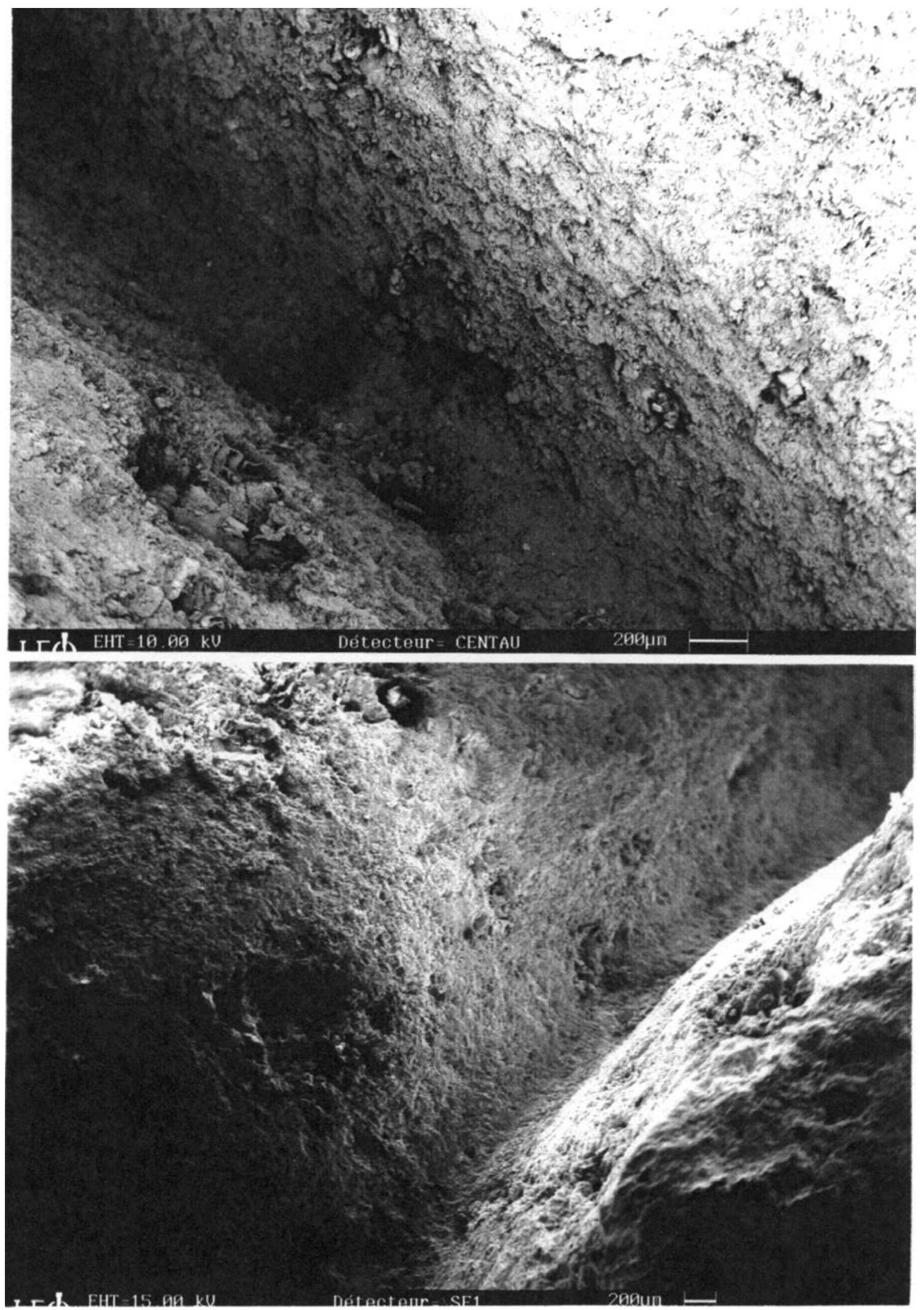

Figure 11. SEM photographs of experimental grooves produced on tuff clasts by retouched cutting edges. 
straight walls and narrow foundations (Fig. 12a), while those made with retouched blanks generally show one straight and one convex, concave or irregular wall, and often have rounded foundations (Fig. 12b). Angles produced by unretouched blanks (Fig. 13) are significantly smaller than those produced by retouched cutting edges. The frequency distribution (Fig. 14) of these values clearly show that they represent statistically distinct populations $(p=0.0001)$.

A way to differentiate grooves produced by either retouched or unretouched cutting edges from those produced by a point is to compare several sections of the same circular groove (Fig. 15). The
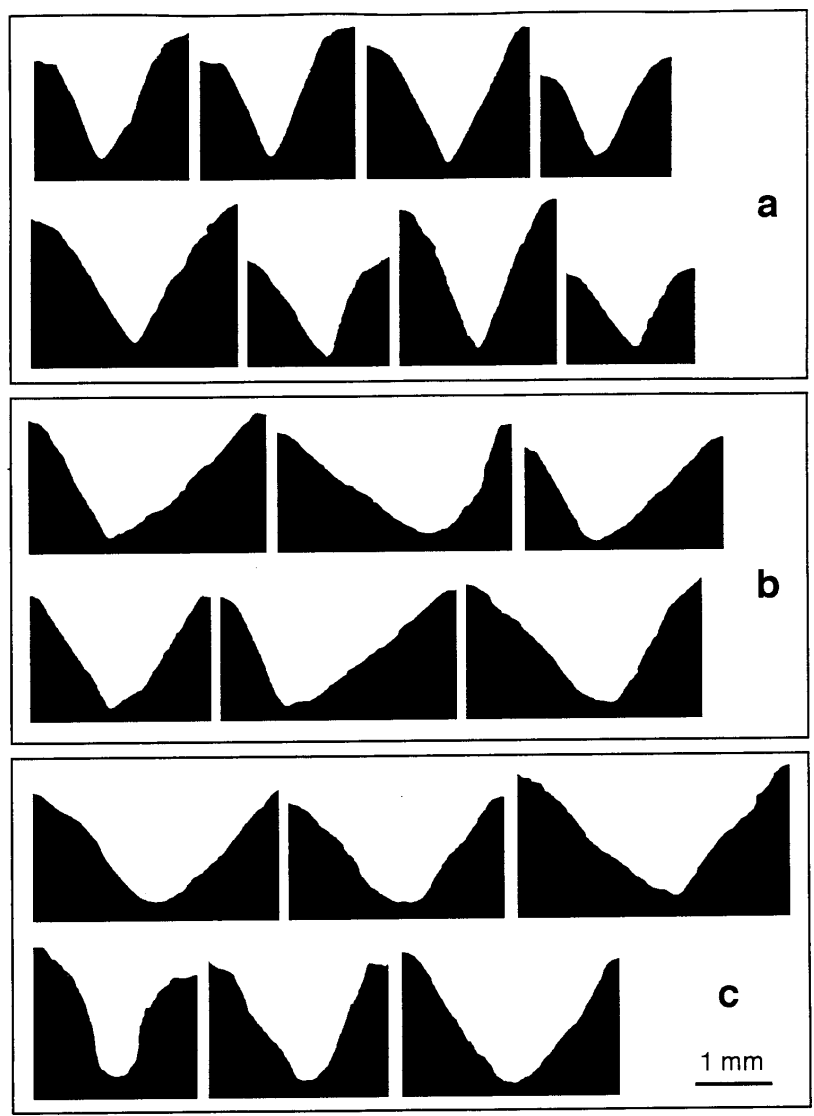

Figure 12. Sections of circular grooves produced experimentally on tuff clasts with (a) unretouched blanks, (b) retouched blanks, and (c) points (burin tips, retouched points, pointed blanks). sections of a groove created by either a retouched or an unretouched blank remain virtually the same along the length of the groove as the elongated morphology of the edge and that of the resulting groove stabilize the tool's motion. In contrast, the sections of a groove created by a point are characterized by stronger morphological variability owing to changes in the orientation of the tool tip during the grooving process.

A sudden change in the direction of the groove without significant variation in the groove width (Fig. 16) is another diagnostic criterion to identify point-made grooves. Experimental grooving demonstrates that only a point can produce such a change, as a straight cutting edge is unable to cut a curving groove without attacking the groove walls and thus increasing its width.

Abrasion

The abrading of tuff clasts on the flat surface of basalt flakes collected at the site obliterates features observed on the natural altered and unaltered surfaces (i.e. superficial deposits, smoothed surfaces,

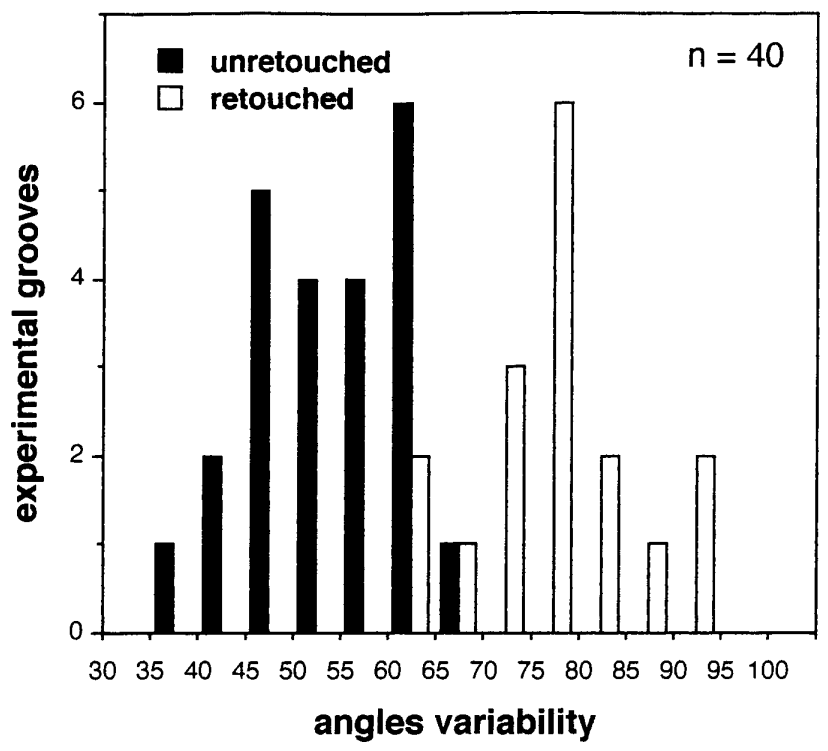

Figure 14. Frequency distribution of the angles formed by the walls of experimental grooves carved by retouched and unretouched blanks.

\begin{tabular}{|lccccccc|l|}
\hline & $\begin{array}{c}\text { No. of exper. } \\
\text { grooves }\end{array}$ & Min. & Max. & Mean & Range & $\begin{array}{c}\text { Std } \\
\text { dev. error }\end{array}$ & $\begin{array}{l}\text { Std } \\
\text { Figure 13. Angle variability } \\
\text { in grooves made } \\
\text { experimentally on tuff }\end{array}$ \\
unretouched blanks & 23 & 39 & 67 & 53.5 & 28 & 7.56 & 1.58 & $\begin{array}{l}\text { fragments by retouched and } \\
\text { retouched blanks }\end{array}$ \\
\hline
\end{tabular}



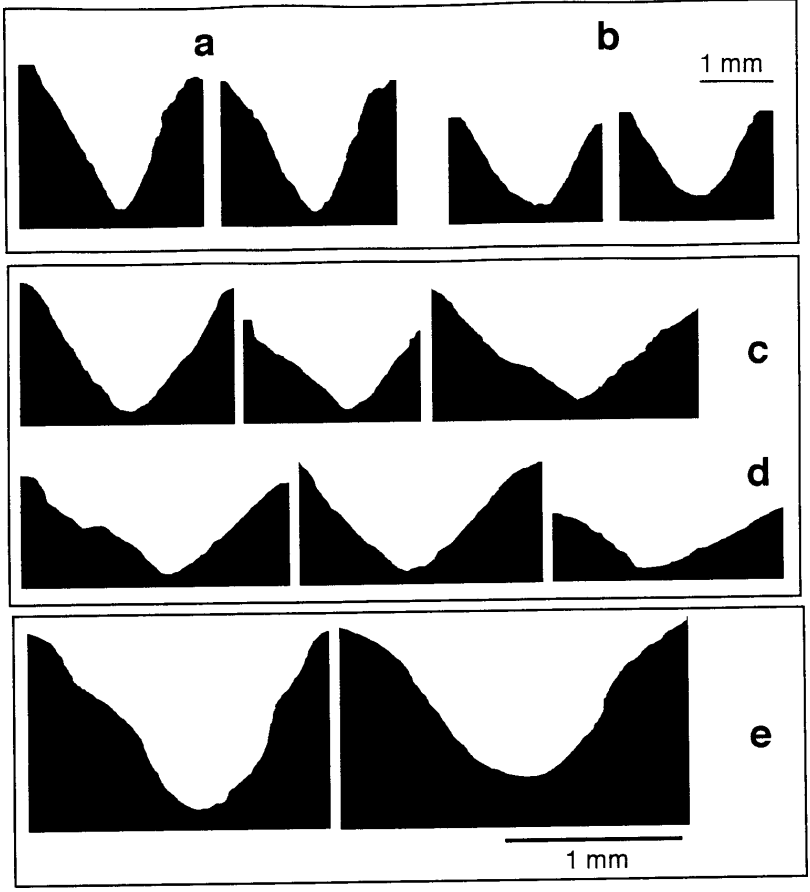

microcraters) and produces rather flat, granulated surfaces with no striations (Fig. 17a). The edges of vesicles intercepted by experimental abrasion show sharp edges. By contrast, vesicles brought to light by erosion or by natural fracturing of the tuff clasts as the latter have rounded to very rounded edges. The development of natural fractures inside the tuff clast is a result of the size and morphology of these vesicles. It is interesting to note that abrading the tuff fragments produces a red stain on the surface of the basalt flake and a relatively large amount of red powder. The reproduction of a facsimile of the figurine produced $0.6 \mathrm{~g}$ of this powder.

Figure 15. Different sections of circular grooves produced by (a) an unretouched cutting edge, $(b)$ a retouched cutting edge, and $(c, d)$ two flint points, compared with (e) two sections of the 'neck' groove on the rear face of the Berekhat Ram figurine.

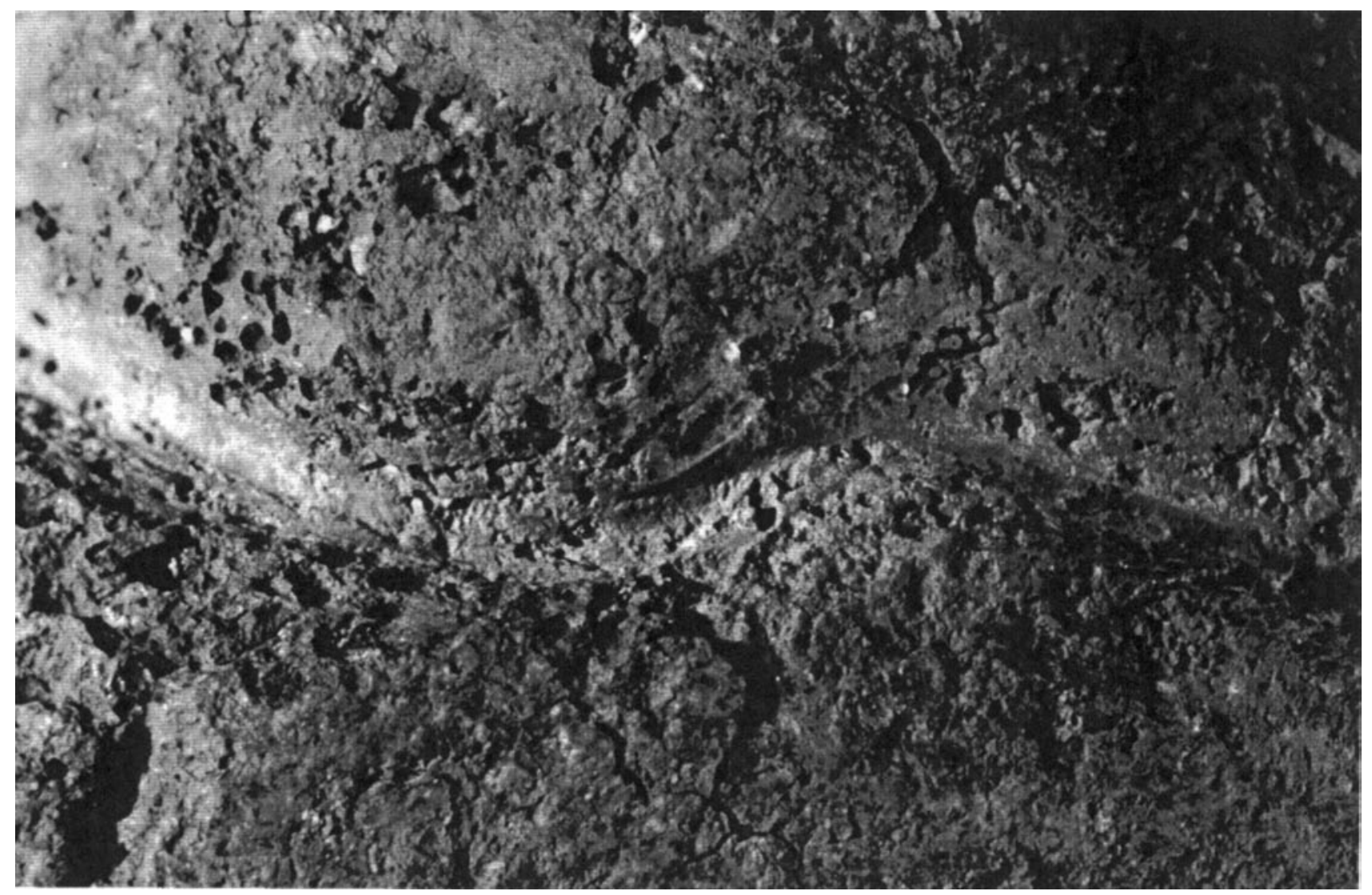

Figure 16. Curve engraved experimentally with a flint point on a tuff fragment (scale $=1 \mathrm{~mm}$ ). 
a

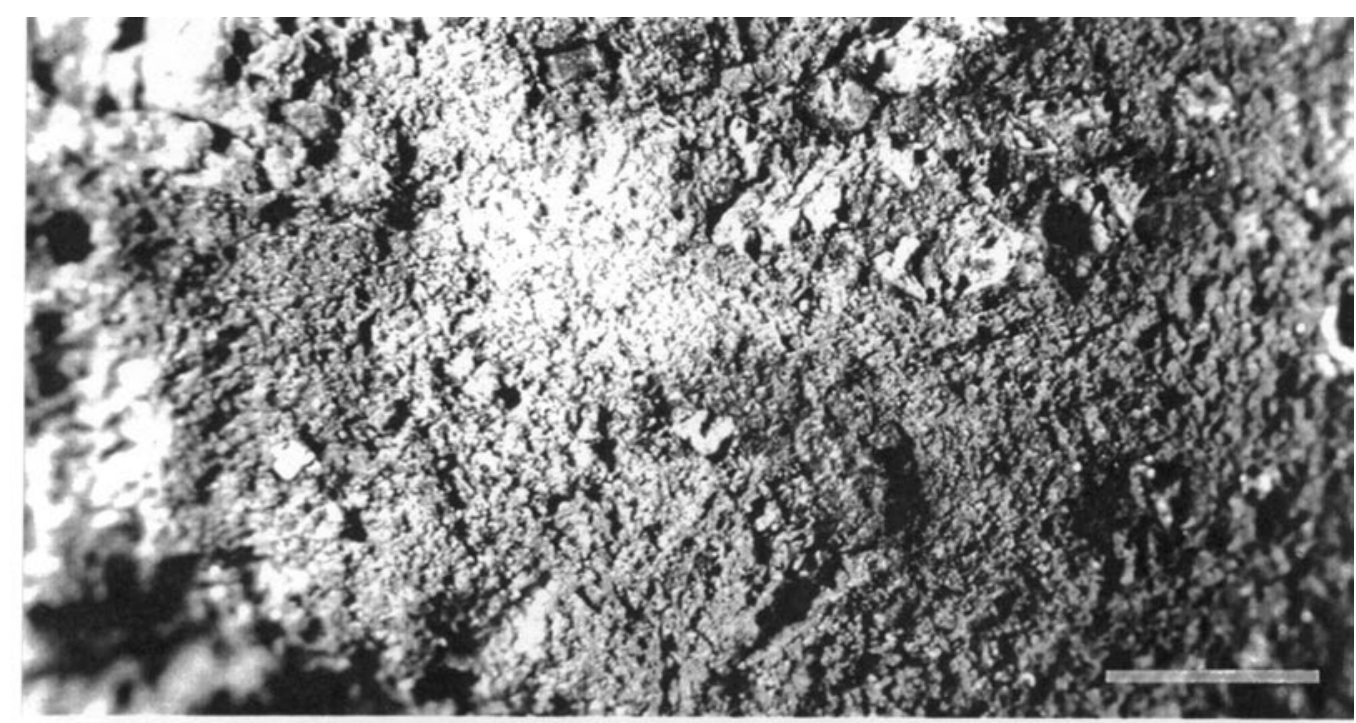

b

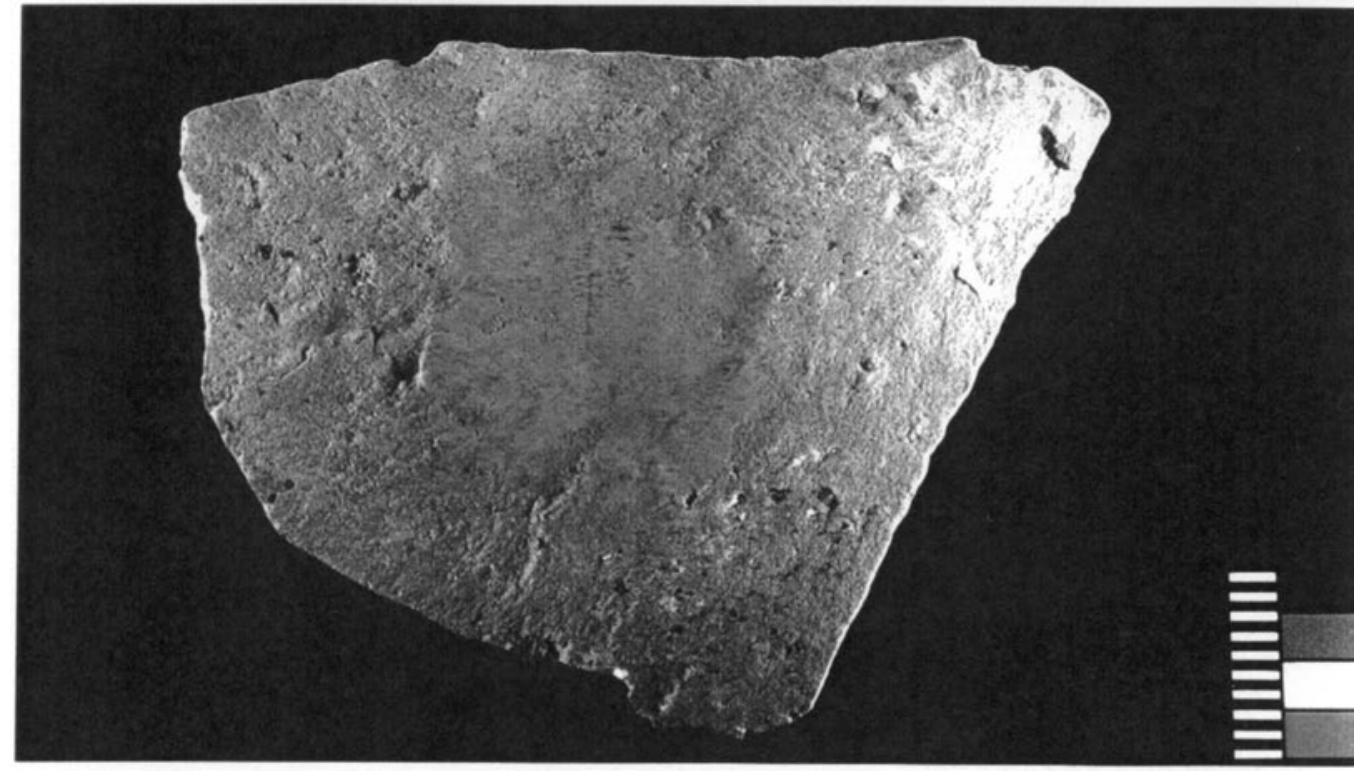

C

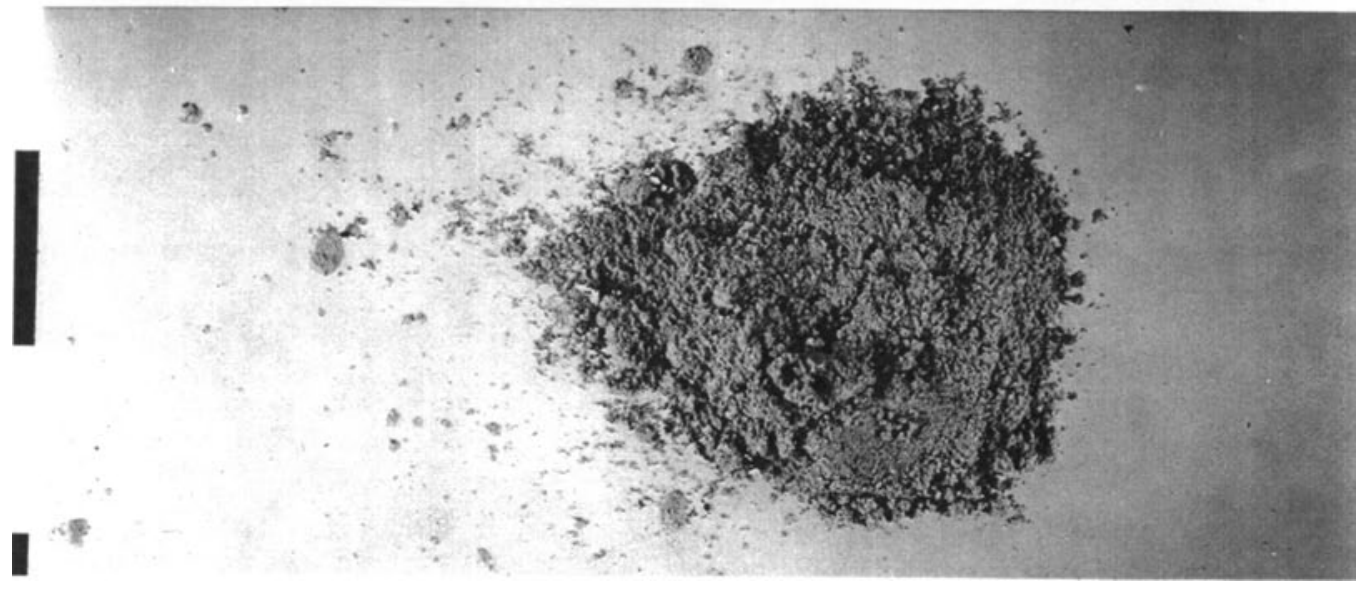

Figure 17. (a) Surface of a tuff fragment experimentally abraded on the flat surface of a basalt flake (scale = $1 \mathrm{~mm}$ ); (b) basalt flake showing a spot of red colour resulting from the use of the object to abrade a tuff fragment; (c) red powder $(0.6 \mathrm{~g})$ produced in the course of the abrasion to carve a replica of the Berekhat Ram object. 


\section{The figurine}

The archaeological specimen possesses a number of features that are reminiscent of those produced experimentally by the engraving or abrading the tuff clasts (Fig. 18). Intentional engraving seems responsible for the neck groove and the two slightly curved grooves on both sides of the object. A short groove produced by engraving has also been carved on the left side of the rear face. This interpretation is supported by the clear morphological differences, visible at a microscopic scale, between the surface of these grooves and those of the adjacent area (Figs. $19-20 \& 22 b-c)$. The former shows a homogenous granulated texture reminiscent of that observed inside grooves made experimentally with a stone tool. Close inspection of the groove foundations reveals the presence of many in situ broken inclusions of different sizes. The area adjacent to the groove has a more irregular and varied appearance where micro- craters alternate with both a rough texture and highly smoothed surfaces on the more exposed areas. As with experimental engraving, vesicles cut by the grooves show rather sharp edges, while those on the neighbouring areas have smoothed funnel-like shape openings.

On the right side of the rear face of the object (Fig. 21a), the neck groove has a morphology that is typical of tool-made engravings, consisting of one straight, vertical wall and one concave, sloping wall, and a narrow foundation. This groove segment also shows a discontinuity in the morphology and direction of the groove, not described by Marshack. It is very similar to the morphology that we produced experimentally when we changed the direction of the tool slightly during the grooving process. The morphology of two sections of the neck groove (Fig. $15 \mathrm{e})$, recorded on the dorsal face near the left end and in the middle of the groove, is similar to that produced experimentally on the same raw material
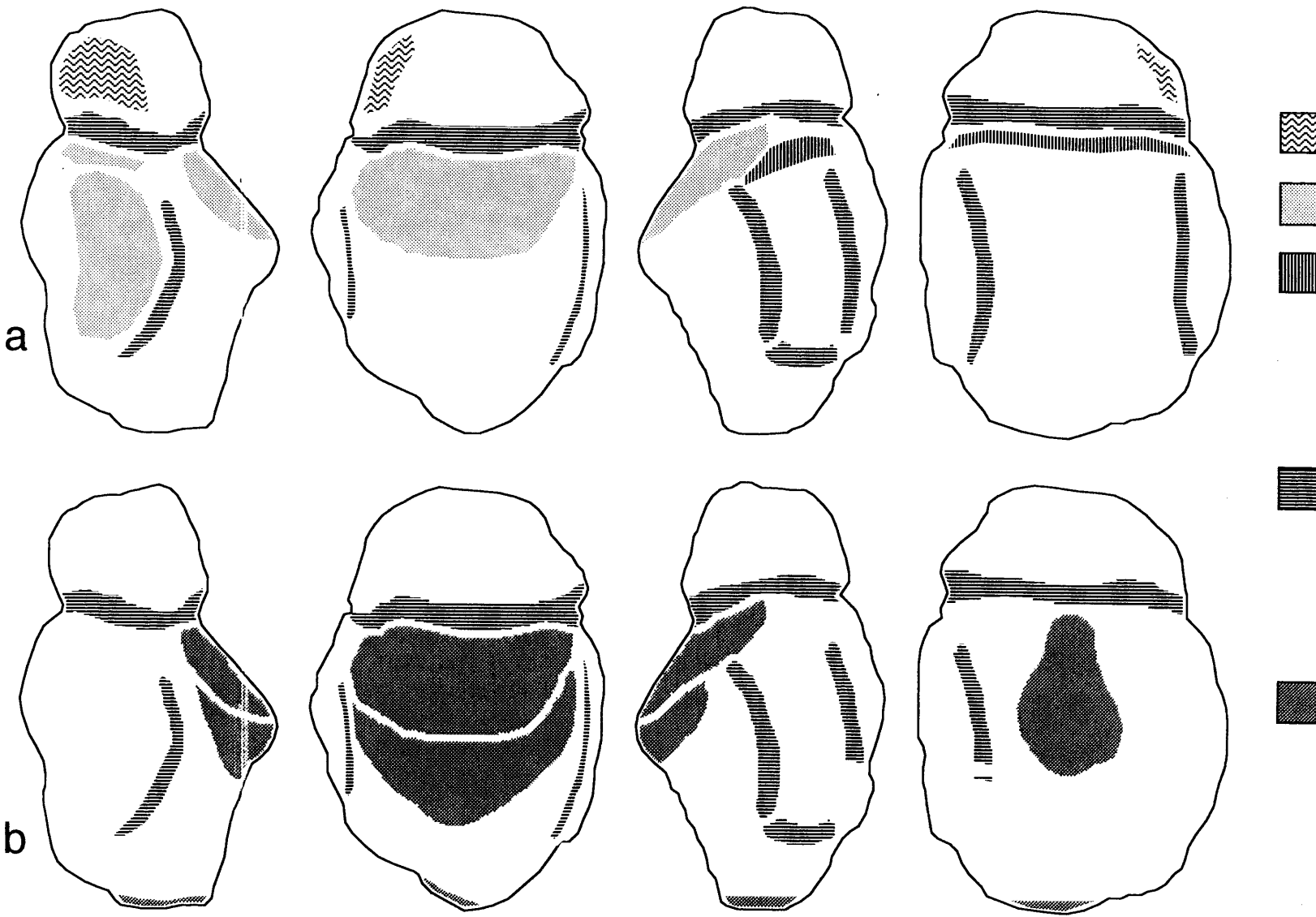

Figure 18. Anthropogenic modifications on the Berekhat Ram object according (a) to Marshack and (b) to the authors of the present study: 1) 'shaping and beveling of the right protuberance of the head'; 2) scraping; 3) artificial smoothing of the lower plan of the neck groove; 4) grooving; 5) possible abrasion. From left to right: right side, front face, left side, rear face. 

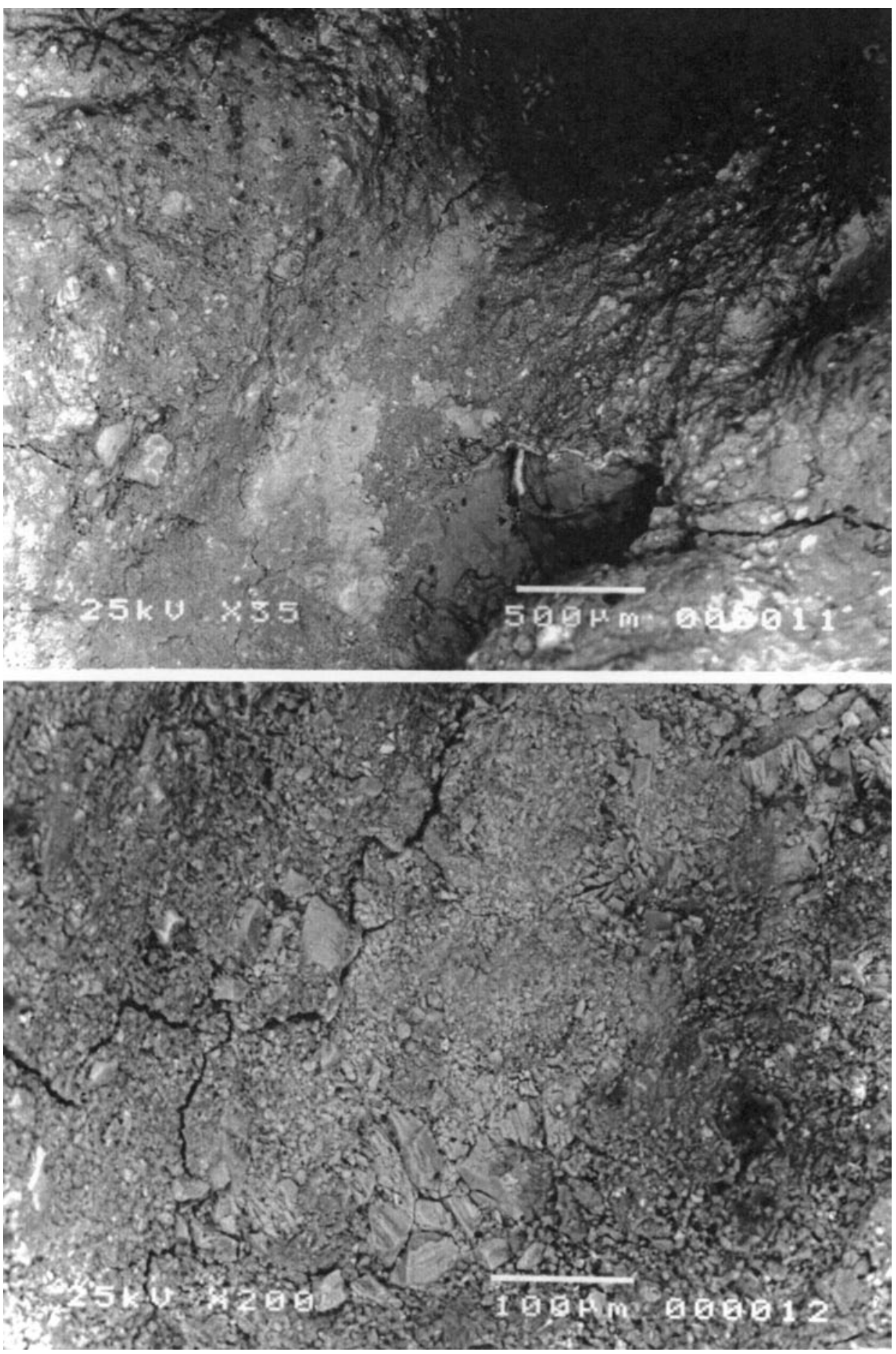

Figure 19. (a) SEM photo of the neck groove on the rear side of the figurine showing the groove section. Notice the difference between the granulated morphology of the groove surface and that of the vesicle intercepted by the groove wall (arrow); (b) detail of (a) showing the foundation of the groove.

with a retouched blank or with a thick point. The morphological variability of the groove section, how- ever, suggests the use of a point instead of a cutting edge. The use of a point is also confirmed by the curved outline of the groove on the left side of the front face (Fig. 21b), which has been shown experimentally to be difficult to produce with a cutting edge. The curved outline of the lateral grooves and their sections also suggest that a thick point was used to carve them. The flat appearance of the two adjacent surfaces defining the 'chest' protuberance on the front face, their surface morphology at microscopic scale, and the way the vesicles are cut by these surfaces suggest that they may be the result of humanly-caused abrasion. This may also be the case for a slightly rounded area on the rear face of the object and for a small, flat plane carved at the base of the object.

In sum, while confirming Marshack's diagnosis of the anthropogenic origin of several features present on the object, our technological reading of the piece differs from that he proposed on several points. We see no traces of the scraping which would have carved the right shoulder and arm, no artificial smoothing of the lower plane of the neck groove, and no intentional modification of the right protuberance of the head. While shallow grooves are present on the left and right sides of the anterior surface one may wonder whether they should be seen as delineating 'arms' (see discussion below). We think, in contrast, that the base of the object was deliberately 
modified by abrasion to create a small flat surface, perhaps to allow the object to stand. These may, however, be considered relatively small differences with Marshack's analysis, as the main point was to ascertain whether the object was modified by hominids.

\section{Discussion}

The combined use of actualistic and experimental data suggests that the Berekhat Ram specimen was modified by humans. These modifications are clear on the 'neck', and less obvious but still detectable on the 'arms' and 'chest'. We believe that we arrived at this conclusion following a path which differs significantly from that followed by Marshack since we developed a methodology to study this object. This methodology is based on the creation of a reference collection of tuff clasts from the site and the microscopic analysis of unmodified fragments and grooves on both scoria and experimentally modified tuff fragments. We then identified a set of diagnostic criteria using a number of different tools and techniques. These criteria are relevant because they are based on two different types of evidence: 1) microscopic analysis of experimental engraving; and 2) morphological and metrical analysis of the groove sections. Finally, we applied these criteria to the analysis of the figurine and found features which closely resemble those produced experimentally. While we cannot logically exclude the possibility that taphonomic processes may exist which can mimic some of the features reproduced by us experimentally and found on the figurine, our comparative approach and experimental research support an anthropogenic origin. The principle of equifinality, however, leads us to observe that it is

Figure 20. (a) SEM close up view of the head of the figurine. (b) SEM detail of the groove on the rear of the figurine showing morphological differences between the groove and the adjacent upper surface.
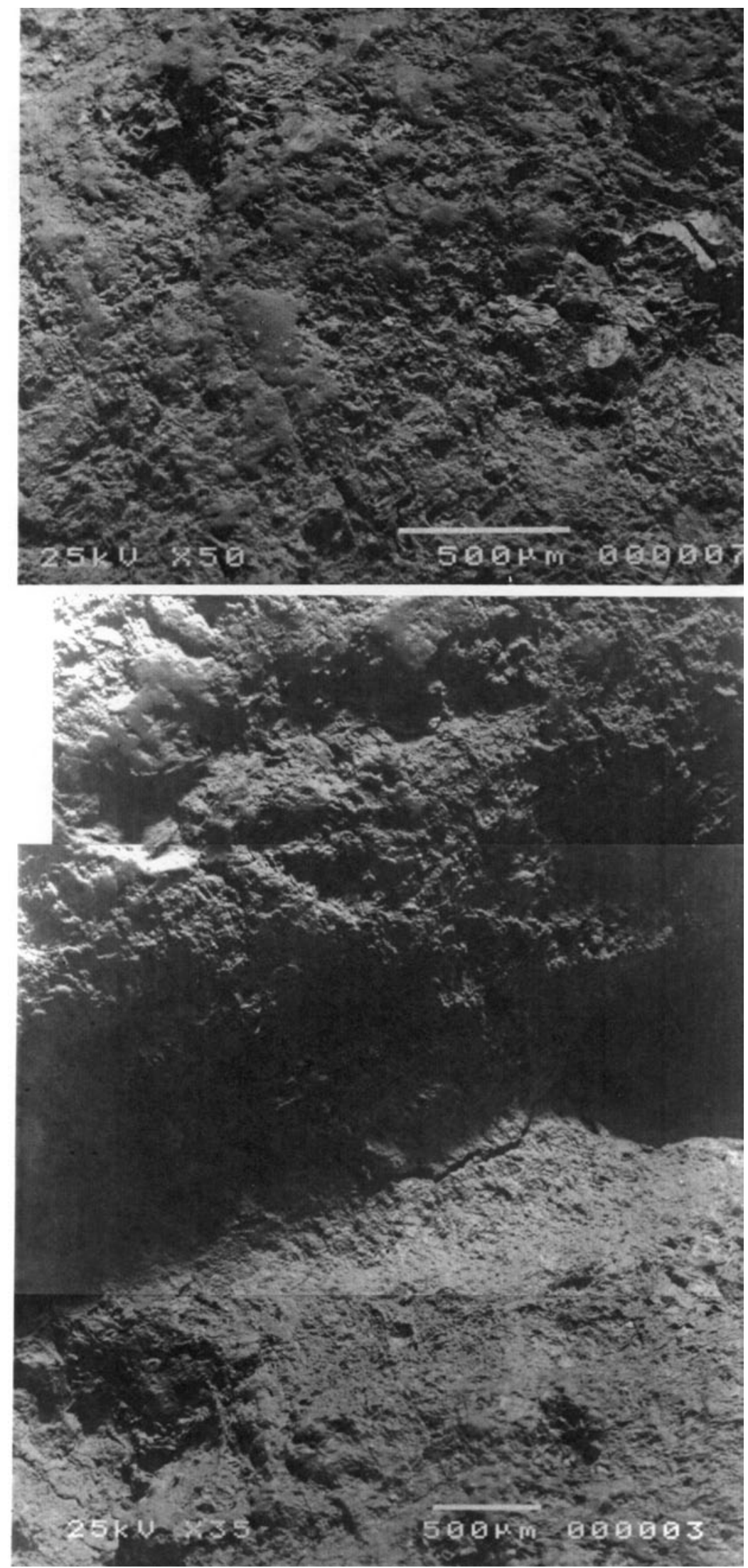
a

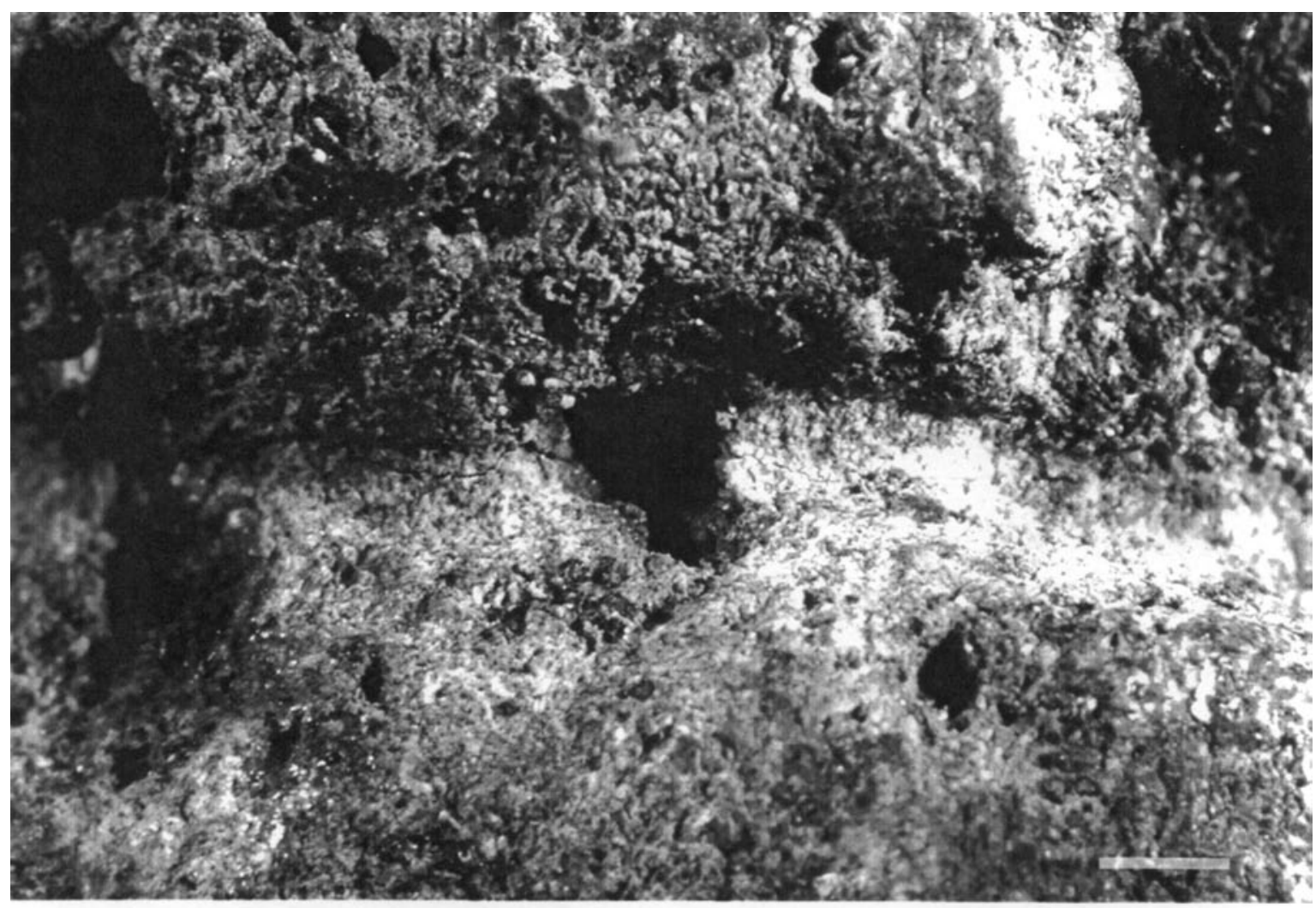

b

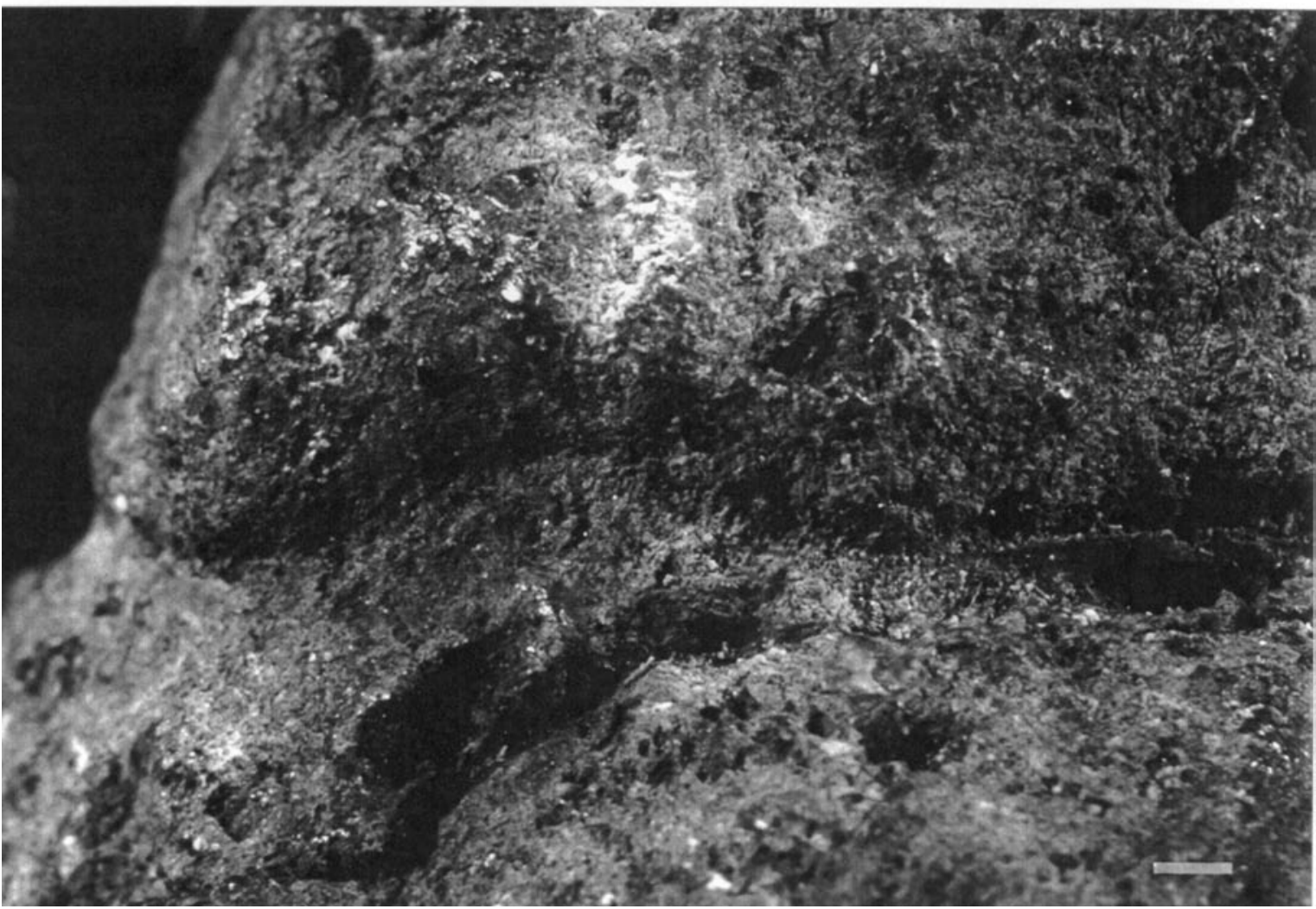

Figure 21. (a) 'Neck' groove on the rear of the figurine showing discontinuity in the groove direction; $(b)$ detail of the neck groove on the left side of the front face. 

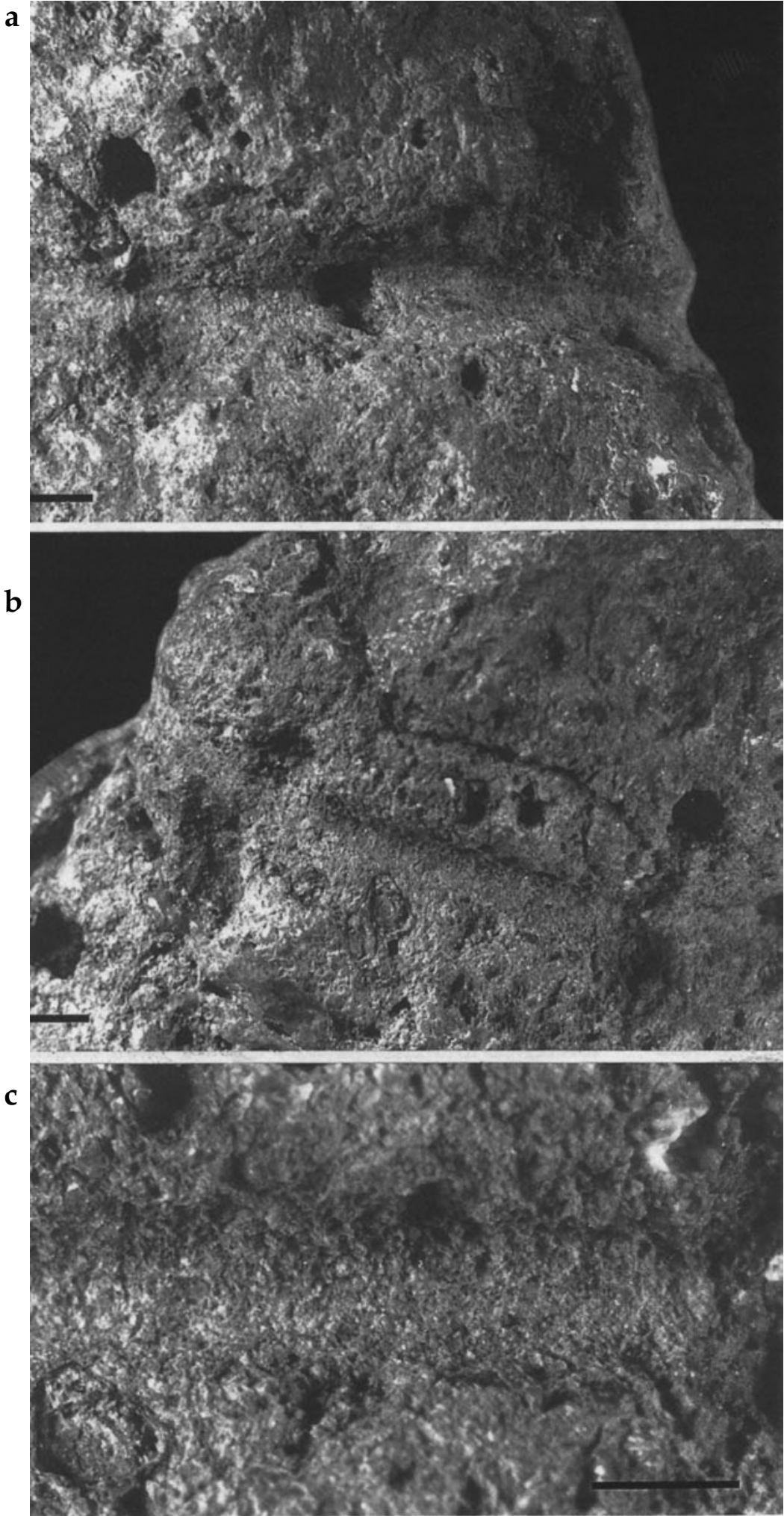

Figure 22. Top: 'neck' groove on the rear side of the object showing a discontinuity in the groove direction, its asymmetrical section, and the presence of superficial striations on the groove bottom. Middle and bottom: notice the granulated texture of the groove surface. Scales $=1 \mathrm{~mm}$. possible that hominids may have produced some of these features by using a technique or procedure that we have not tested experimentally.

The objective of our research was to determine if the Berekhat Ram figurine was the product of human or natural processes. Now that our results suggest that the specimen is anthropogenic it is appropriate to consider what its function may have been and, by extension, its possible implications for the origin of human symbolling in the Near East. The carving of a circular groove on a tuff fragment does not provide in itself any proof that it is a purposely-made three-dimensional carving, nor can it even be taken as evidence of symbolling. The identification of an anthropogenic groove on a tuff fragment only demonstrates that Acheulean hominids used their stone tools on a variety of raw materials. This is consistent with data from the use-wear analysis of several stone tool assemblages of this period (Keeley 1980; Anderson-Gerfaud 1990). The discovery of a number of worked wooden objects from Europe (Movius 1950; Adams 1951; Oakley et al. 1977; Thieme 1997) and the Near East (Belitzky et al. 1991) further attests to their ability to work with a wide range of materials. All of this data is compatible with what we know about Acheulean stone tool technology. The use of different reduction sequences and different types of raw material to produce a varied tool kit seems well documented (Wynn 1993; Bar-Yosef \& Goren-Inbar 1993; Schlanger 1996).

The ethnographic record (Chase \& Dibble 1992, 48) demonstrates that grooves can be made on different types of objects for a number of functional reasons. The groove in itself only confirms that Acheulean stone tools provide us with a poor and rather biased image of a more complex technical system that is based on the modification of a wide range of raw materials. It is therefore clear that the problem of the interpretation of the Berekhat Ram 
figurine lies more in the elucidation of the other, less obvious, anthropogenic modifications found on the object than in the clearly human produced circular groove. Our results suggest that areas of the figurine other than the circular groove were purposely modified by humans. In particular, as already suggested by Goren-Inbar and Marshack, two elongated ' $u$ ' shaped grooves could have been made to indicate the arms of a human body and different areas were possibly modified by abrasion, perhaps to suggest breasts.

The recognition of a human body in this specimen is, however, problematic. It ultimately depends on the criteria one employs to identify a human figure in a carved object and the level of uncertainty that one is willing to accept as part of this 'identification'. This is a recurrent problem in the study of prehistoric carvings which appear relatively abstract to modern observers but which might be the schematic rendering of depictional representations (see discussion in Rosenfeld 1977 and in d'Errico 1995a). So far, however, none of the authors who has described the Berekhat Ram specimen as a figurine have formalized the morphological attributes that they have used to make this proposition, nor have they made reference to criteria adopted by other scholars (e.g. Rosenfeld 1977; Duhard 1993a,b) for identifying Upper Palaeolithic human representations.

This leads us to the second problem in identifying a human body in this object: the lack of comparable material. A large number of Upper Palaeolithic human representations, like those from Gönnersdorf (Bosinsky 1991; Bosinsky \& d'Errico in prep.), would not have been described as human figures without the discovery of many other more detailed figures at this and other contemporary sites. In this respect, the point made by Noble \& Davidson (1996) that the Berekhat Ram object was identified as a human figurine because it resembles the Upper Palaeolithic specimens is a justifiable criticism in the sense that it would be an error to use, consciously or unconsciously, the Upper Palaeolithic record to support the symbolic interpretation of a carving produced in a clearly different cultural context and quite possibly a different cognitive context as well.

We doubt for all the above reasons that it will be possible to reach an agreement about the subject represented. One can even wonder if this is really important. What matters here is that some of the engravings and abrasions are not necessarily consistent with the hypothesis of a functional use of the object but rather appear as the product of a pur- posely made representation. This is the case for the 'arms', whose symmetry and design are difficult to account for by a functional explanation. The significance of this desire to represent something is the real problem here. If representational, as suggested by our technical analysis, this object could have had a merely iconic meaning (i.e. it was carved to resemble the subject for which it stood: Peirce 1931-35), or a symbolic meaning. In this latter case, one should assume that an arbitrary link was established by the Acheulean craftsperson between the morphology he or she produced or modified and an image or concept to which this referred.

Many archaeologists working on anatomically modern human (AMH) societies would seek to resolve this question by studying the context of similar and different representations, their geographical and chronological distribution, their morphological and technological variability, and so forth (Ucko 1962; Renfrew \& Bahn 1991; see examples in Renfrew \& Zubrow 1994). Of course, this is difficult here as we are dealing with a single object.

Moreover, these same scholars when investigating the meaning of symbols emerging from the archaeological record would implicitly or explicitly assume that symbol systems were used in the past in a way that is not qualitatively different from the way they are used in modern or traditional societies. To make the same assumption in our case would mean employing circular reasoning, as the goal in assessing the implications of this find for the origin of symbolling would be inherent in the postulate.

To avoid this pitfall, we employ a lower level of inference, and assume only that the basic neuromotor constraints conditioning the technical behaviour of the Berekhat Ram engraver are not qualitatively different from those affecting the modern engraver in her/his replicative experiments. Within the framework of this assumption, the technological analysis of the object and its experimental reproduction are seen as complementary means to evaluate the technical skills of the Palaeolithic craftsman, to understand his aim, and to assess the cognitive implications and the overall significance of his work.

Experimental reproduction of the carving shows that the chosen raw material played a major role in the somehow crude appearance of the representation and that it would have been difficult to achieve a higher level of realism on a piece of raw material of this type and size. If it was the result of a repetitive behaviour, the engraver must have been perfectly aware of the level of depictional accuracy possible in working a tuff clast. We must then conclude that 
whatever the subject of the depiction, the engraver deliberately made the choice of a small support and did not need a more detailed representation to recognize the depicted subject. Such recognition cannot be achieved without a relatively elaborated level of abstraction. If iconic, the representation is far enough away from the subject for which it stood to suggest that the Palaeolithic craftsperson or observer did not need a closer resemblance to establish a meaningful link between the two. If symbolic, we are forced to admit that the arbitrary link between the meaning and its material appearance was solid enough to be established on a visually rather cryptic incarnation of the symbol.

Now that we have made explicit the possible implications of our results, the question becomes how to deal with something like the Berekhat Ram engraved tuff in the context of what we know (and do not know) about the origin of symbolism. In our view, there are two possibilities to consider. One is that the origin of symbolism is essentially a radical, qualitative change in human cognition. This is the model that many semioticians, linguists, psychologists and neuroscienticists advocate. If it were the case, then there are two options: either symbolism is the result of a biological change that occurred at a precise moment in human evolution, and from that time onward modern humans became symbol-users; or symbolling is the result of a cognitive change within a particular cultural system (Chase in press; d'Errico et al. 1998c), a change, to use a familiar analogy, in the 'software' but not in the 'hardware' of the brain. For the first option, Darwinian selection would be the most likely course. For the second, the cognitive shift could relate to concomitant changes in the social system and in the need, for example, for a more sophisticated means of recognizing the members within and between social groups as those increased in size (Gamble 1982; Gilman 1984; d'Errico et al. 1998c).

Another possibility is that the emergence of symbolling does not correspond to an abrupt change but to a more gradual process (Duff et al. 1992). This view is contrary to the position held by a number of authors (e.g. Byers 1994; Mithen 1996a; Noble \& Davidson 1996; Mellars 1996). It agrees, however, with current models on the origin of spoken language (Liebermann 1995; Tobias 1998). This scenario implies that at some point in time there existed hominid societies that were only 'partially' symbolic or where the system of symbolling was less complex than that of modern humans or where, at least, symbols played a less important role in the way the mind constructed reality. This proposition may appear heretical to some, but we nonetheless need to ask if the emphasis on abrupt biological or cultural change is not influenced by disciplines that have concentrated their efforts on the study of symbol systems of modern humans, and have paid little attention to the construction of theories to explain the transition between non-symbolic and symbolic worlds. In other words, an abrupt change, of biological origin, is without doubt the most comfortable explanation for those interested in the functioning of symbolic systems rather than in their origin.

Revealing the existence of this pitfall, however, is not in itself proof of the gradual emergence of symbolic capacities. Gradualist arguments suffer from serious difficulties. First, it is difficult to imagine what a 'partially symbolic' human society would look like. Secondly, we should avoid creating 'arguments for design', i.e. only when the feature or organ is fully formed can its utility explain its development.

Archaeologists who study the evolution of human intelligence need to look for ways of testing these hypotheses using the archaeological record rather than being influenced from the beginning by one hypothesis or another. Results emerging from archaeological inquiry may present a strong challenge to the reigning paradigms. They may even constitute the base upon which to build alternative theories, providing better answers when tested against the available archaeological evidence. What is surprising, and in some ways stimulating, when we examine the possible archaeological indications of the adoption of symbolic thinking, is the fact that a large portion of the evidence coming to light in the last few years seems to contradict the dominant theories of the origin of symbolling.

The view of symbolling as being a cultural epiphenomenon of the emergence of AMH with their biological superiority is contradicted by, for example, the absence of evidence of similar behaviour among the first modern humans of North Africa and by the existence of personal ornaments made and worn by the last Neanderthals of Europe (d'Errico et al. 1998a,c; Zilhão \& d'Errico 1999; in press). Even accepting, which is debatable, that Neanderthal use of symbols was a consequence of their contact with moderns humans, the fact that they were indeed capable of such an endeavour clearly contradicts the hypothesis that symbolic thinking results from a biological change.

The view of symbolling as a phenomenon destined radically and rapidly to change cognitive 
mechanisms and, in consequence, the everyday life of a symbolling humanity, is in opposition to the gradual increase in possible symbol-bearing indicators such as utilized and notched pieces of ochre, and in the number of engraved objects uncovered in the African Middle Stone Age. This view is also contradicted by the sporadic presence of a few engraved pieces and some ochred and pierced shells among early modern humans or late Neanderthals in the Near East. If symbolling really represents that qualitative shift that many theoreticians and archaeologists believe it to be, why does it appear in the archaeological record in form of scant, isolated, though clearly significant objects, as is the case in the Near East (e.g. Qafzeh and Quneitra)?

In conclusion, while new archaeological evidence challenges a merely biological origin for symbolic capacities, the material expression of this behaviour in pre-modern societies is difficult to identify and does not allow us, so far, to discern clear patterns of development.

The Berekhat Ram carving clearly represents a supplementary challenge to dominant paradigms on the evolution of human cognition. A conservative attitude would insist on discarding this evidence simply because it does not fit in with any of the current models on the emergence of symbolic thinking. For this reason some scholars might feel that the object does not deserve any further inquiry. The history of archaeology, however, demonstrates that what is rejected today as too idiosyncratic to be incorporated into any reasonable theory can, tomorrow, become an accepted fact that may allow us to gain a better insight into the evolution of humankind. Obviously, the uniqueness of this find constitutes a major handicap in evaluating its significance and only future discoveries will show whether it remains a puzzling mystery or is among the first examples of representational art. Whatever the future, our results seem to indicate that this object cannot be dismissed as the result of natural processes and should instead be considered as a purposely modified object, of possibly iconic or symbolic meaning, and not qualitatively different from more recent examples of symbolic expression.

\section{Acknowledgements}

We thank Nathan Schlanger and João Zilhão for their stimulating critical reading of the manuscript. This research was made possible by financial support from the French Institute of Culture and Technology, the University of Pennsylvania, the Jewish Community
Centers of Greater Philadelphia Israel Programs, and Jean Auel. We also thank the LEO representative in France for allowing Francesco d'Errico to use their impressive SEM (model 435VP) free of charge. We owe a special debt of gratitude to Naama GorenInbar for allowing us to study the Berekhat Ram figurine and for helpful discussions during the preparation of this work. Our thanks also go to Debby Hershman, curator, and Naomi Korn, assistant curator of prehistory at the Israel Museum, Jerusalem, for facilitating our research; to Martin Kessel, Hebrew University, for his support of our project; to Naomi Bahat, Faculty of Agriculture, Hebrew University, for her assistance with the SEM microscopy; to Leslie Hale, Mineral Sciences Department, Smithsonian Institute, for supplying us with information on volcanic geology; and to Dodik Ben-Ami for his invaluable help during our visit to the Berekhat Ram site and for providing the flint used in our experiments. Finally, we thank the Israeli Antiquities Authority for giving us permission to conduct our research.

Francesco d'Errico
UMR 5808 du CNRS
Institut de Préhistoire et Géologie du Quaternaire
Avenue des Facultés
33405 Talence
France

April Nowell
Department of Anthropology
University of Pennsylvania
33rd and Spruce Streets
Philadelphia, PA 19104-6398

USA

\section{Comments}

From Ofer Bar-Yosef, Department of Anthropology, Peabody Museum, 11 Divinity Avenue, Cambridge, MA 02138, USA.

It is comforting to know that the findings of the original observation by the excavator (N. GorenInbar) and the first microscopic study (A. Marshack), suggesting that the Berekhat Ram figurine is of anthropogenic origin and is not a natural accident, are now confirmed by the methodologically rigorous study of the authors. Perhaps this is a good lesson for critics and journal editors to be more prudent in the future and to demand the careful re-examination of the original item before publishing a negative 
review. This is not to say that criticism should be abolished. The questioning of accepted 'facts' is the way in which science strives for further advancement. It would, however, be to the benefit of archaeology to embrace certain basic standards of the Natural Sciences. Archaeologists and editors who accept negative reviews need to demand that the authors of such papers rely on the results of experimental investigations and not solely on the study of texts and printed photos. Similarly, scientists who submit dating results obtained by new techniques to an archaeological journal should be required to demonstrate that their techniques were tested on objects or fossils of known age as was pioneered by $\mathrm{W}$. Libby for $14 \mathrm{C}$ some 50 years ago.

The authors of this article, like most of us, struggle with the meaning of those isolated Lower and Middle Paleolithic 'artistic' expressions found in the archaeological records prior to the so-called 'creative explosion' of the Franco-Cantabrian region. The fact that the latter is a unique geographic phenomenon within the larger world of Homo sapiens sapiens cultures is crucially often forgotten. While the Upper Palaeolithic in Eurasia has all the signs of a true techno-cultural revolution, there is no need to deny that some early hominids were able to demonstrate planning-depth. The required savoir faire (e.g. Schlanger 1996), in the case of Middle Palaeolithic chaînes opératoires, only shows that we who classify these lithic products would perhaps be in trouble if asked to produce a series of thin, symmetrical Levallois points. Discoveries such as the wooden spears from Schöningen (Thieme 1997) from some $400 \mathrm{ka}$, indicate that organic finds in pristine condition substantiate the view that the capacities of past humans exceeded those of chimps. Notably, what hampers archaeologists and palaeoanthropologists in understanding the Lower and Middle Palaeolithic is, as already mentioned by Tooby \& DeVore (1987), that there is no model to use as an analogy for early hominid behaviour.

Resorting to either the chimp or the modern forager model does not serve us well in understanding the biological, technological and cultural evolution of Homo ergaster, Homo erectus, Homo heidelbergensis, and Archaic modern humans. The original impetus for this evolutionary trajectory could have been, as proposed by Deacon (1992), the change in shape and increase in size of the braincase of Homo erectus. Comparing the technological achievements of hominids during successive time transects from 1.8 ma through $8 \mathrm{ka}$ BP reveals periods of gradual change - sometimes very slow - punctuated by major revolutions (i.e. the MP/UP transition or the Neolithic revolution).

From Angela E. Close, Department of Anthropology, University of Washington, Seattle WA 98195-3100, USA.

D'Errico and Nowell have produced an exquisitely argued article on the Berekhat Ram object (whether or not it is a 'figurine', it is definitely an 'object') that is probably the most useful thing to have been written on this subject to date. To my mind, it is certainly the most convincing. Using a carefully documented experimental approach, and using very high-power microscopy to compare their experimental results with the object itself, they demonstrate that the object has most probably been modified by human action. They are careful to point out that they cannot prove that this occurred, or that it was done with the techniques that were used in the experiments. Such caution is commendable, but I, at least, am now prepared to accept that the object is an artefact, owing some of its physical characteristics to human action. This is the most important contribution of this article. If they had shown that the form of the object could be accounted for by non-human agents, then we could all have forgotten about it or continued to ignore it.

D'Errico and Nowell's outlines of the major schools of thought concerning the beginnings of symbolic behaviour and of the history of interpretations of the Berekhat Ram object are perceptive. They provide a clear picture of the way in which scholarly discussion has proceeded in the absence of relevant data and without the possibility of recovering relevant data: largely by assertion and counter-assertion.

D'Errico and Nowell begin their discussion of the object with extreme caution, pointing out that, 'The carving of a circular groove ... [cannot] even be taken as evidence of symbolling'. A couple of pages later, however, we are told that, 'the engraver must have been perfectly aware of the level of depictional accuracy possible in working a tuff clast'. I still do not see any reason to link the Berekhat Ram object with 'depiction' or any other type of symbolic behaviour. It follows from this that I certainly cannot see a figurine of a woman in the object. In fact, the drawings in Figure 18b remind me more of a penguin (doubtless tales had been passed down through the generations since their ancestors left South Africa), and the photographs in Figure 1 remind me more of a phallus. 
I also do not see any reason to refer the object to the food-reproduction-shelter side of life, but these are not the only possibilities. I can invent several untestable scenarios which would imply levels of cognition, or even of social complexity, that are unexpectedly high for a quarter of a million years ago, but these are no more helpful than the untestable 'figurine' scenario. While the object is most probably an artefact, without any evidence of a 'purpose', I do not assume that the modifications were necessarily purposeful. When our minds are elsewhere, our hands will frequently manipulate objects that simply happen to be there, not for any purpose. This is, of course, another untestable hypothesis.

I am less optimistic than d'Errico and Nowell and expect that, for me, the Berekhat Ram object will remain 'a puzzling mystery'. Because of the methodological sophistication of their approach, however, it is a mystery that will not now go away, and we may long expect to see the object commandeered by both 'sides' of the symbolling debate.

From João Zilhão, Instituto Português de Arqueologia, Av. da Índia 136, 1300-300 Lisbon, Portugal.

This article is of the utmost importance in developing a methodology to address the thorny issue of whether certain apparently 'carved' objects found in Lower and Middle Palaeolithic contexts are natural or anthropogenic. On the basis of the results of the experiments described by d'Errico and Nowell, their conclusion that the Berekhat Ram 'figurine' has been intentionally modified seems entirely warranted. They go on from this finding to discuss whether an iconic or symbolic meaning can be assigned to the piece. Although the speculation is legitimate in the face of previous interpretations, I see nothing in their evidence that would support such an assignment.

The strongest argument presented in favour of the representational hypothesis is the symmetry and design of the 'arms' (p. 144). Previously, however, the authors had wondered whether the shallow grooves present on the left and right sides of the anterior surface should indeed be seen as delineating arms (p. 140). They explicitly contrasted the dubious finality of those grooves with the obvious abrasion of the base of the object to create a small flat surface. The inferential step made on page 144 where they state that their technical analysis suggests the 'arms' most likely are a purposely-made representation - is justified on the basis that the grooves are difficult to account for by a functional explanation.

Two logical points can be made against this argument. First, a functional interpretation is not explicitly discussed and refuted and, therefore, simply stating that other explanations are possible does not necessarily entail the rejection of function. Second, even if function could be set aside, that would not necessarily imply the acceptance of the hypothesis of a purposely figurative or symbolic representation. It would still be necessary to show that those grooves are not an unintended, indirect result of intentional or random gestures executed in the framework of a manipulation of the object for functional purposes.

That such a manipulation may indeed have been functional is indicated by the observation reported by d'Errico and Nowell that abrading the tuff fragments produces a relatively large amount of red powder. As they also remark, utilized pieces of ochre are commonly found in Lower and Middle Stone Age contexts. Thus, the hypothesis naturally comes to mind that the Berekhat Ram 'figurine' was reduced for the production of pigment and that its grooved and abraded areas, although undoubtedly anthropogenic, are by-products of such a reduction process.

Therefore, the possible iconic or symbolic meaning of the object is not demonstrated, even if some of its features are shown not to be the result of natural processes. Furthermore, its very small size, the fact that it is possible to formulate alternative explanations for the grooves, and the degree of uncertainty that, with present knowledge, is inherent to any interpretation of this 'figurine', make any conclusions premature. Contrary to the possibility suggested in the article's last sentence, my reading of the evidence is that the Berekhat Ram 'figurine' is indeed qualitatively different from more recent, unequivocal examples of symbolic expression.

I fully concur, however, with d'Errico and Nowell's rejection of a biological mutation, exclusive to the lineage of modern humans, as the mechanism behind the sudden appearance of symbolism. Such a view would be refuted if obvious symbolic or iconic objects were to be found in an Acheulean context. The opposite, however, is not true: that none have been found so far does not carry the implication that the biological model is vindicated. In fact, the widespread evidence for the practice of burial and the use of ochre in the Middle and Lower Palaeolithic suggests the presence of symbolic thought among anatomically archaic humans, as does the production and use of ornaments by late 
Neanderthals.

That it was only in Upper Palaeolithic times that such a capacity for symbolic thought materialised in the production of objects with a symbolic value must, therefore, have a social explanation, as d'Errico and I extensively argued elsewhere. That this was a relatively sudden process only implies a biological explanation if one assumes that cultural evolution proceeded through a Darwinian phyletic gradualism. The whole of human history is there to show that such an assumption is not warranted.

From Steven Mithen, Department of Archaeology, University of Reading, Whiteknights, Reading, RG6 2AA

It is curious that our understanding of such an immense topic as the evolution of human symbolic behaviour appears to hinge on the study of such diminutive and visually unimpressive pieces of stone as the claimed figurine from Berekhat Ram. I very much welcome this article, as a detailed study of this type was an urgent requirement in light of the attention recently paid to this 'figurine'. Marshack's (1997a) publication was a redundant piece of work, for without the type of experimental and comparative study undertaken by d'Errico \& Nowell, his claims were of limited value; one can only think that the then editors of Antiquity were seduced by his colour photographs. D'Errico \& Nowell have now provided as detailed a study of this artefact as seems possible - although I suspect that others engaged in this specific type of work may nit-pick about their methods. But to me they appear quite systematic and comprehensive. I do, however, have some concerns about their interpretation and their general comments about symbolic evolution.

The division of work in this area into the two camps of 'discontinuists' and 'gradualists' is too simple. My own work (Mithen 1996a) is placed in the former camp, even though it explicitly argues that the building blocks for symbolic thought were gradually laid down throughout the earlier Palaeolithic. Without that gradual evolution of human cognitive capacities any seemingly sudden appearance of symbolic behaviour could not have been possible. Hence I think it more appropriate to characterize my own position as gradualist, with regard to the cognitive capacities that allow symbolic behaviour, and discontinuist as regards the manifest appearance of such thought, this arising from one further step in the gradual evolution of such capacities. In this regard, it is significantly different to the type of discontinuist position that argues that symbolic behaviour emerged from one chance mutation, which suddenly and dramatically transformed the human mind. I suspect that others similarly characterized as discontinuists might also argue that their position is rather more subtle, sophisticated or complex than is the impression given by d'Errico \& Nowell.

A second point to raise about the discontinuity/gradualist division is that this does, of course, depend upon one's perspective. When considered within the time-frame of primate evolution (c. 65 my), or even human evolution since the common ancestor (c. $5 \mathrm{my}$ ), a 'gradual' appearance of symbolic thought during the last 250,000 years might be characterized as a rather sudden discontinuity. Similarly, when looked at from the perspective of the duration of human life history and generation span, a 'discontinuous' appearance of symbolic thought within, say, a 10,000-year period of the Late Pleistocene, would have been a very gradual change, with ample time for selective pressures to work.

I found d'Errico \& Nowell's distinction between a biological origin of symbolic thought and one locating this in a 'cognitive change within a particular cultural system' (p. 145) to be rather too vague to be of any great use. The brain/ computer analogy which suggests that the former is about changes in hardware and the latter about changes in software is in fact a rather unhelpful analogy. Each person's neural networks are continually being remoulded in light of experience, this occurring at a particularly substantial rate and extent during childhood and adolescence. In other words, changes in cultural behaviour can change our biology - the density and networking of neurones in specific parts of the brain are altered, and if one must use the brain/ computer analogy, this is perhaps more like a change in hardware. The resulting positive feedback between changes in culture and biology are potentially immense and, hence, one might expect there to be periods of rapid behavioural and cognitive change.

There are two important issues to raise with regard to the possibility of partial or proto-symbolic capacities. The first is the idea expressed so effectively by Clive Gamble, that the origin of symbolic behaviour would have been like throwing on a light switch - once something is symbolic everything is potentially symbolic. Hence a phase of protosymbolism is likely to have been so rapid as to be archaeologically invisible. I am not sure if the lightswitch analogy provided by Gamble is correct, but it is certainly compelling.

A second issue concerns the cognitive basis for 
symbolism and whether this is necessarily the same in archaic and modern humans - an argument I have previously raised, in response to some rather extreme propositions by Bednarik about claimed early symbolic artefacts (Mithen 1996b). One of the difficulties in making comparative studies between living primates is whether similarities in observed behaviour reflect similarities in cognition. Consider primate vocalizations and human language. These have at least one critical feature in common - they both communicate information by audible means. But few scholars today would credit chimpanzees with any linguistic ability: chimpanzee vocalizations - although having similarities to human utterances - are likely to have a quite different cognitive basis to human language. My own interpretation has been that chimpanzees are simply rather clever at using general-purpose learning mechanisms for acquiring associations between a symbol and its referent (Mithen 1996a). But this does not stop what they are doing as looking like a proto-linguistic ability. Another case in point is whether chimpanzees have a theory of mind capacity - whether they are able to appreciate that another individual has beliefs and desires that differ from their own. After an initial rush of enthusiasm that this was the case in light of various behavioural observations, such as claimed acts of deception, several primate specialists are now more cautious, suspecting that such behaviours may simply arise from chimpanzees being very clever 'behaviourists' (see Povinelli 1996; Whiten 1996; Mithen 2000). In other words, while lacking a theory of mind capacity, chimpanzees are remarkably adept at picking up behavioural cues about how another individual is going to act in the future, which to us appears as if they are engaging in 'mind reading'. The same can be seen within modern humans: two individuals can be asked to solve a mathematical problem and reach the same answer in the same time, but one might be essentially relying on rote memory, while the other may be using various mental computations.

The point I am making is that just because we find artefacts from the early Palaeolithic record which we decide have an iconic or symbolic status, we cannot automatically conclude that such objects had been made using the same cognitive processes as used by modern humans for symbolic thought, or indeed an early version of those processes. Objects such as the Berekhat Ram figurine and Bilzingsleben incised bones may indeed be symbolic, but may derive from general-purpose learning mechanisms in the early human mind, quite different to whatever cognitive processes for symbolism we use today. In this regard, the rarity, variability and lack of chronological development of such objects may reflect the limited extent of symbolic thought that such general purpose intelligence can generate - in the same way as chimpanzee symbol-use is so limited (to a threshold of 200-300 words) because they do not have a proper linguistic ability.

A final issue I must raise is that the writing and arguments within this article are sometimes rather less stringent than is desirable when discussing such controversial subjects. Although the authors had quite rightly set out to evaluate whether the incisions on the object from Berekhat Ram were natural or made by early humans, the object is always referred to as a figurine - this surely is what is trying to be established and a neutral term (object, artefact) would have been far more appropriate. Similarly, the 'discussion' section is immediately preceded by a sentence that refers to the object having being modified by 'hominids', and then begins with an almost identical sentence but which refers to 'humans'. As some scholars, such as Davidson and Noble, have thought it appropriate to restrict the word 'human' to a symbol/language-using hominid, (which I personally think is unnecessary), I think d'Errico \& Nowell should have been much more cautious and consistent with regard to their use of such terms.

There is at least one enormous, and seemingly quite unjustified, leap of interpretation made within this article. D'Errico \& Nowell not only claim that some of the engravings and abrasions are non-functional, but that they are also 'the product of purposely made representation' (p. 144). I can see no justification for this claim: I can see no evidence to support their claims for 'symmetry' and 'design' of so called 'arms'. There are other leaps of interpretation throughout the article of a less serious but still rather irritating nature. On p. 145, for instance, Neanderthals are described as not only having made body ornaments, but also having worn them. There is no evidence for the latter and it is a separate issue from their manufacture. My own feeling is that if Neanderthals made/acquired items of body ornament, having been influenced by modern humans, they did not really know what to do with them. This, however, opens up issues beyond the scope of the current article. Finally, I was concerned about the statement on p. 146 that 'A conservative attitude would insist on discarding this evidence [i.e. the Berekhat Ram artefact] simply because it does not fit in with any of the current models on the emergence of symbolic thinking'. That would not, of course, be 
a conservative attitude but one of utmost academic irresponsibility and I cannot imagine any archaeologists currently engaged in studying symbolic evolution, even the most ardent discontinuist, giving this artefact less than the full attention it deserves.

In summary, this is a fine article with regard to the systematic study of the Berekhat Ram figurine. I am happy to accept d'Errico \& Nowell's conclusions that early humans made minor modifications to a small lump of rock. I cannot see any evidence to support the idea that the resulting artefact had a representational or symbolic status. I suspect that the modifications were for utilitarian purposes and that we should not for a moment be surprised that such artefacts exist in the archaeological record. As regards symbolic evolution in general, I feel that the current debates, and the issues that must be addressed, are already rather more complex and sophisticated than d'Errico \& Nowell allow for within their text.

From Thomas Wynn, Department of Anthropology, University of Colorado, Colorado Springs, 1420 Austin Bluffs Parkway, P.O. Box 7150, Colorado Springs, CO 80933-7150, USA.

As I read it, d'Errico and Nowell make three arguments: the Berkehat Ram figurine is a modified object, it probably had a symbolic function, and it challenges interpretations that stress a late and abrupt acquisition of symbolic activity in human evolution. Their careful analysis has persuaded me that the object has been modified by a stone tool. Their argument for the 'neck' groove is compelling, though their arguments for the arm grooves and abrasions are not as strong, and probably would not be convincing in the absence of the neck groove. I am not, however, convinced by their argument for symbolism, and as this is the crux of the argument for significance I will focus my comments here.

My first reservation lies with the argument for depiction. Eliminating a utilitarian explanation for this object may, in fact, be impossible. To their credit, d'Errico and Nowell have not simply taken recourse to 'unidentified' function, which is a common fallback position for most archaeologists. Instead they explore the implications of a non-utilitarian role. In doing so, however, they omit a third option, which given the depictive poverty of the object seems to me just as reasonable. Could not the object have resulted from biomechanical fiddling (someone passing time with a stone tool and a pebble)? We may be too quick in concluding that material objects from the past resulted from purposive action; much of modern human action is not purposive - why should the past be different?

My second reservation concerns the nature of the argument. D'Errico and Nowell set up an interesting and perhaps unintended paradox. If the object is depictive it is not a very accurate depiction, and therefore must be symbolic in the narrow sense rather than iconic (i.e. it does not really look like a woman, so we, and presumably the artisan, must decide that it represents a woman). The paradox derives from a corollary argument, and its evolutionary implication. Increasing veracity in depiction supplies decreasing evidence for symbolism. The Vogelherd horse, for example, is an accurate depiction of a horse, so it can be iconic and not symbolic. There is something counter-intuitive here. If the Berekhat Ram figurine were an accurate depiction of a woman, would they discount it as evidence for symbolism? Their argument would make sense if the object were one of an array of less and less iconic objects, like the figurines of the Upper Palaeolithic. We accept the stylized versions as symbolic because they grade into contemporary examples that are more iconic. We do not have this luxury for Berekhat Ram. It is effectively all by itself, not the end point of an array of decreasingly iconic forms.

My final reservation struck me at first as entirely trivial, but it does bother me and I have never seen it discussed. The object is so very small! Its real size appears to be about two-thirds the size of the reproduced image. Moreover, the raw material is very coarsegrained. This combination of features strikes me as inconsistent with a depictive role, at least if modern carving is any guide. Yes, modern people carve diminutive figurines, but not in coarse-grained materials, such media being reserved for large images. There seems to be a kind of detail-to-size ratio in modern carving that may well have a perceptual basis. Furthermore, there is nothing very 'micro' about the rest of material culture found in this time period, making the diminutive size of the object even more unusual.

Despite my reservations concerning the depictive nature of the Berekhat Ram figurine, I do agree with d'Errico and Nowell on an element of their third argument: there has been little attempt to generate hypotheses about what a pre-modern life world might have been like (though see Noble \& Davidson 1996; Byers 1999). Worse for the interpretation of this object, there has been no attempt to construct theoretically-grounded hypotheses of what pre-modern depiction might look like. Without such rigour we are reduced to informed guessing. Cogni- 
tive science, development psychology, semiotics and art history should be able to provide the basis for such hypotheses. For example, reference to perceptual psychology might provide insight into the size/detail issue (Gibsonian psychology's use of 'affordances' comes to mind), or judicious use of developmental psychology might provide insights into the development of depiction (though simple recapitulationist arguments are almost certainly unwarranted).

Given our current level of understanding, this object is likely to remain an enigma. If it demonstrates anything, it is that evidence rarely, if ever, speaks for itself.

From Alexander Marshack, Peabody Museum of Archaeology and Ethnology, Harvard University, 11 Divinity Avenue, Cambridge, MA 02138, USA.

It is profoundly satisfying and unexpected to have $\mathrm{d}^{\prime}$ Errico and Nowell write that they have, in some measure, 'validated' my finding that the Berekhat Ram figurine was intentionally modified, with the implication, however, that their validation is better than the original because they had experimented with volcanic tuff, established analytical criteria, and used scanning electron microscopy. The authors then address the possible relevance of the figurine for an understanding of the human capacity. These represent different areas of inquiry and discourse, so I address them separately.

First, the analytical problems and data they discuss are not quite as described by the authors. It should also be obvious that modification of a natural form does not occur at the edge of a tool but in the perception and intent of the tool holder. It is apparently the belief of the authors and of those they cite that questions of 'intent' must remain a black box. This is not, as I shall show, entirely true. The original Berekhat Ram analysis was conducted, incidentally, not to test whether it had been modified, but to test, if it had been modified, the nature of the perceptual, problem-solving and cognitive processes that may have been involved. The inquiry, therefore, occurred at an entirely different level than was explored by the authors. Mere 'validation' would have been of interest primarily to that portion of the archaeological community that does not believe in an 'archaic' human capacity for complex symbolling. Such 'validation', by its very nature, exists at the lowest possible level of archaeological inquiry. By contrast, the possible cognitive, perceptual, and problem-solving processes that may have been involved in the early modification of natural forms would be of generic relevance to an evolutionary inquiry into the human capacity.

It was with recognition of the generic problem that this researcher had, for instance, validated the presence of symbolling at the Middle Palaeolithic site of Quneitra in Israel, documenting a complex early capacity for 'abstraction' before the migration of anatomically modern humans into Europe (Marshack 1995c). Microscopy had been used in the Quneitra analysis not merely to 'validate' intentionality but to explore the nature of some of the cognitive and perceptual processes that may have been involved. It was with concern for such cognitive problems that I have widely investigated the nature and range of the early human capacity for symbolling (Marshack 1985). It was in the same context that I studied different early modes of modifying and using natural forms: in the Middle Palaeolithic at Tata and Arcy-sur-Cure; in the Upper Palaeolithic at Monpazier and La Marche and in the sanctuary caves; in the Epipalaeolithic, Early Neolithic and Bronze Age of the Levant; and in the Middle Stone Age of Africa at Border Cave.

One reason for these broad studies is that the modification and use of natural forms is crucial to any study of early symbolling. The beginnings of personal adornment, one of the supposed hallmarks of early symbolling, usually consisted of (according to the taphonomically skewed record) a modification of natural forms, such as sea shells, animal teeth, other skeletal parts and fossils. My early microscopic study of the animal-tooth beads from the Châtelperronian site of Arcy-sur-Cure was an inquiry, therefore, into the nature of the cognitive processes involved. One hand had held, turned, and oriented a tiny bead, while the other hand held the tool and incised a categorizing modification. That microscopic study would prove important to the Berekhat Ram analysis since it demonstrated that a presumably 'archaic' human could use a two-handed, visuallymediated, right/left hemisphere capacity to perform a highly specialized categorizing modification - on non-utilitarian objects. I had previously found a comparable Neanderthal capacity in my microscopic study of the modification of a mammoth-tooth lamelle and the engraving of an image on a tiny fossil nummalite from the site of Tata in Hungary. It was clear that a two-handed capacity for modifying non-utilitarian artefacts was present quite early. My Arcy analyses, however, indicated far more complex cognitive processes than a mere production of beads.

The full set seemed to include a group of learn- 
ing pieces. This set (Fig. 23a-c) would certainly not have been received in trade or exchange. But the grooves on even the better beads, such as Figure 23c, did not quite meet and would have been difficult to secure for long with a string. They may, as a result, have required a use of mastic. The use of mastic to bind thongs during hafting was later documented in the Mousterian (Shea 1988). It was also apparent that the sequence of bead production at Arcy went beyond an incising of grooves; it required planning and the acquisition and use of different materials and skills and a knowledge of the time, place and context for use of the beads. The complexity of these disparate processes in time and space required evaluation and mediation by the frontal and prefrontal lobes, extensive implicit and explicit knowledge, and highly evolved visually-mediated motor coordination. It was the accumulation of such findings over many years (cf. Marshack 1996b) that underlay my decision to undertake a microscopic study of the Berekhat Ram figurine. Among the Israeli materials I had found evidence for a modification of natural forms that was highly varied, if not as numerous as the modification of natural forms that I had found among the Upper Palaeolithic mobiliary materials and caves of Europe. The Arcy and Israeli studies led me almost at once to recognize the mismatch in the neck groove that encircled the Berekhat Ram figurine. The clearly incised groove in the front did not meet the groove in the rear, either at the right or left of the figurine (Marshack 1997a, 332). This was microscopic evidence of intentionality without a need for experimentation with volcanic tuff or a use of scanning electron microscopy. That single set of preliminary findings had suggested a process of modification on the figurine that had proceeded as a sequence of discrete, different instances of categorizing decision-making, 'depiction', and problem-solving. It was not mere validation of intentionality that was, thereafter, important, but the discriminative, categorizing nature of that sequence of intentionality.

There were other preparations for the Berekhat Ram analysis. My studies of the modification of natural forms had indicated that the production of an 'anthropomorphized' image often involved the addition or highlighting of a morphological or anatomical attribute (head, neck, eyes, nose, mouth, legs, arms) or the addition of a cultural marker: a belt, a necklace, a bracelet, or a coiffure, often indicating a
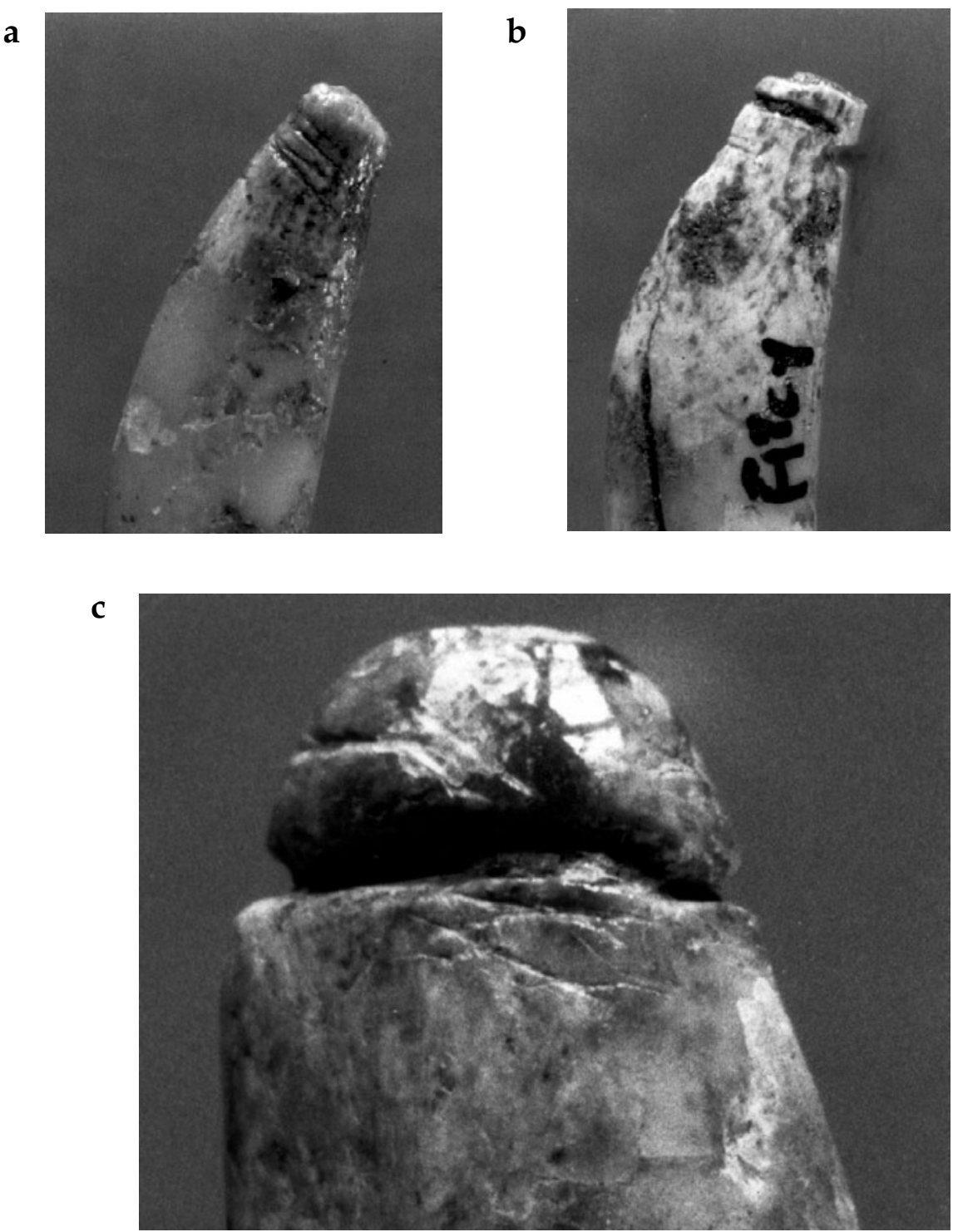

Figure 23a-c. The encircling grooves around some of the Châtelperronian beads from Arcy-sur-Cure, France, indicating that the grooves did not always meet or form an adequate groove and would probably not have served to secure a string. 
'female' without the need of anatomical features such as breasts or vulva. These cultural additions clearly often constituted a sexual marker. Even the relatively realistic female images of the Upper Palaeolithic were so marked. The supposedly naked 'Venus' figurines of the Gravettian often had body bands, bracelets, necklaces, skirts, and coiffures or caps, all originally made of perishable materials. The figurines were not, therefore, naked. Such cultural markers were as much an indication of the 'female' as the better-known aspects of anatomy and morphology (cf. Marshack 1991b; Gvozdover 1995; Soffer et al. forthcoming). It was with a knowledge of such processes that I had investigated the possible modification of the Berekhat head to enhance an appearance of 'managed hair'. The natural form was, in fact, already strongly suggestive of a head with hair (Marshack 1997a). For d'Errico and Nowell the possibility of such categorical enhancement is clearly 'problematic'. However, my microscopic study of the descending plane of the protuberance on the right side of the head indicated that its surface was different from the granulated surface above that descending plane and different from the surface on the head itself (Marshack 1997a, fig. 6). I doubted that the groove of the neck, intentionally incised and arced to abut that protuberance, would have, or could have, left the protuberance in its original state. A slight modification would have enhanced the already existing appearance of hair. It was also clear that after a burial of 250,000 years, surface erosion and taphonomic change had occurred on the outer surfaces and protuberant areas, more than they had in the protected declivities and grooves which clearly showed microscopic evidence of tool use. D'Errico and Nowell's laboratory experiments in incising and scraping tuffic lapilli did not involve experiments with the differential processes of erosion and taphonomic change that had clearly occurred in areas of the figurine. In my paper I indicated that the area of an early spalled flake on the outer surface of the head (Marshack 1997a, 330) had been profoundly discoloured and eroded so that it matched the original granular surface and colour of the head. The protuberance suggesting 'hair' and the flat shoulder plane on the right side were, in that sense, 'outer' surfaces and would, therefore, have differed in their erosion and texture from the more protected declivities. They would not have shown the same clear evidence of tool modification as found in the protected grooves. ${ }^{1}$ I have other minor differences with d'Errico and Nowell. In my paper I demonstrated by colour photography that the material beneath the outer surface of the figurine was a high red (Marshack 1997a, 331). D'Errico \& Nowell's subsequent experiments with abrasion to produce a red powder confirmed that finding, though they do not cite the original finding (Marshack 1997a, 331). Additionally, it should be clear that if abrasion had been used to shape the chest or breast, as suggested by the authors, it would have produced a high red chest or bosom, just as the process of incising and scraping the neck would have produced a red grooving. That possibility has its own ramifications, one of which would have been the creation of red powder, perhaps then available to cover the entire figurine or to be used by a female during a 'female' ritual, perhaps also involving the figurine (see Marshack 1996b). Female figures in the Upper Palaeolithic were often marked with ochre (at Willendorf, Laussel, etc.); I have suggested that early depictive images may, in fact, have been made not as 'depictions' but for differing modes of symbolic use, including ritual overmarking. It was not the capacity to make an image but the capacity to properly use an image that was probably of ultimate cultural relevance. It is clear that the Berekhat Ram figurine will continue to raise questions that go beyond a mere validation of intentional modification. I wish, however, to address more important issues.

In my Berekhat Ram paper I documented the fact that water-worn pebbles and flint forms could be turned into an 'anthropomorphic' figure with a simple encircling line. Such modifications of a natural form are ubiquitous in human cultures and they probably represent a process that long preceded the creation of fully depictive representations. The use of an animal phalange or metapodium to represent the torso of a woman has been documented from Spain to Siberia, at times with a schematic overmarking of anatomical or cultural motifs though sometimes without (Gimbutas 1989, figs. 3(4), 91 \& 92a; Olsen 1996, figs. 6-9). Artefacts of this type were excavated from an Early Bronze Age level at the town of Jericho in the Levant. Phalanges, metapodia, and animal incisors were used to represent the 'female' even in the Upper Palaeolithic (Jelinek 1975, 336; Marshack 1991c, 391; Gvozdover 1995, 181-5; Lorblanchet 1999, 134, 135). A Siberian shaman will cut a simple forked branch to create, without modification, the image of an ancestor or spirit. This forked stick may later be touched with paint or be decorated with a necklace or feather. Such shamanic creations would not persist archaeologically, and if by accident of preservation they were preserved they would surely not pass d'Errico \& Nowell's 'logical' 
criteria (or those of Ucko, Rosenfeld, or Duhard, cited by the authors) for recognition of an anthropomorphic form. Nor would phalange or metapodial figures pass as anthropomorphic according to their criteria. Yet such modification and use are important modes in almost all preliterate cultures and they are crucial for any understanding of the beginnings of symbolling. There are, however, even more important considerations. I have for long argued that the early use of beads probably arose because there was a prior use of strings and twined cords as necklaces and as ties for the hair. Hanging a pendant bead on such a string would not, therefore, have been the 'origin' of personal adornment; it would merely have modified an extant process. It is the bead, however, that appears in the archaeological record and therefore promotes the presumption of a dated contextual 'beginning' for personal adornment.

It was with knowledge of such processes that, while studying the figurine, I suggested a possible Acheulean indication of 'managed hair', an idea not discussed by the authors. A suggestion of hair was, in fact, already present on the original natural form and this appearance would have been heightened during the incising of the neck grooves. The process of incising an arcing neck groove on the front face, with a slight intentional rounding of the protuberance, would have been enough to enhance the suggestion of managed hair. But my suggestion for possible managed hair did not come simply from my microscopic study of the arcing groove of the neck, the plane of the protuberance, or my knowlege of modification (cf. Marshack 1997a, 331). When handling the figurine, one at first merely sees a 'lookalike' form. While turning and studying the figurine over many days I found myself increasingly confronted with a sequence of perceptually discrete, intentionally produced categories - head, neck, arm, front, back, bosom, possible hair. It was during this process that a conceptual and perceptual deconstruction began. It became clear, by the sequence of changing 'intent' - the delineation and the positional separation of these categories - that they were probably cultural and possibly, therefore, 'named'. Questions of this type cannot be addressed or investigated by the high magnification of scanning electron microscopy, by recourse to logical deduction, by a priori demurers, or by a consensual citation of other authors. They are questions that must be investigated both analytically and with some knowledge of symbolling processes. ${ }^{2}$ It was not, therefore, the possible validation of a modification that was being investigated in my analysis, but the nature of modification, and the nature of what seemed to be culturally recognized Acheulean categories.

The Acheulean productive process and my analysis were both, therefore, 'cognitive'. The left hand turning and orienting the artefact, the right hand performing the categorical incising and scraping, would together have been involved in a sequence of frontal and prefrontal lobe evaluation and mediation while creating differentiated perceptual categories, a process that involved different types of implicit and explict knowledge (cf. Marshack 1997a, 336). While d'Errico \& Nowell's 'validation' acknowledges the presence of many of these differentiated categories, their analytical concern was not with the possible relevance of these categories, but with proof of intentional modification. Even after the modifications have been 'validated', however, the primary generic questions remain. Arguments from probability do not adequately address questions concerning the complexity of a sequence of intentionality.

These suggestions raise questions about crucial limitations in the use of scanning electron microscopy, experimentation, and a priori logic in the study of early symbolling modes. I note this because it was with precisely the same extraordinarily careful experimentation to establish analytical 'criteria,' and with a similar use of scanning electron microscopy, that d'Errico prepared his doctoral dissertation in order to test and invalidate this author's claim for the existence of notation in the Upper Palaeolithic. Since he was primarily interested in the technological problem of determining how incised cross-sections were produced, he never bothered to investigate the nature of notation, how a notation might function, or how one could determine the presence or absence of notation except at the very low level of cross-sectional analysis. As a result, d'Errico chose an incorrect sample for study, apparently because the artefacts were incised. He then declared that he could find no notation in his incorrect sample and that notation, therefore, did not exist in the Upper Palaeolithic. It is important to note that this 'invalidation' utilized precisely the same careful, exhaustive descriptions of technology, precisely the same careful, exhaustive descriptions of experimentation, statisticized graphics, and precisely the same syntax of science, logic, deduction and arguments of probability, as now appear in the Berekhat Ram analysis. Yet d'Errico was in error. The error was not in the experimentation and the derived 'criteria', or the seeming logic, but in their use. I had begun my own research by studying the nature of notation. In my 
reponse to d'Errico I indicated that I had previously studied much of d'Errico's analytical sample and had found that it did not contain notation, according to my knowledge of 'notation.' One artefact in his sample (Ro48), however, was clearly notational both according to my analysis and to d'Errico's own description of it; nevertheless, that artefact was incorrectly illustrated and interpreted by d'Errico despite use of SEM (Marshack 1996a, figs. 1-3). Ro48 was never again mentioned except to indicate that it was unique and therefore aberrational. All notational artefacts are, however, statistically aberrational within the enormous Upper Palaeolithic corpus and most of the important early examples of symbolling are, in fact, also statistically 'unique' (see my examples above). It is not their statistical uniqueness that is relevant, but the analytical evidence for their cognitive complexity. D'Errico began to study a proper sample, when, years later, he found to his surprise that notation did, after all, exist (d'Errico \& Cacho 1994). He described this subsequent 'validation' of notation in precisely the same terms and with precisely the same careful and exhaustive descriptions of technology and experimentation, scientific syntax, logic, and arguments of probability, he had earlier used to 'invalidate' the presence of notation.

These facts are important not because they indicate prior error - one always grows and changes in research - but because d'Errico \& Nowell's current schematic renderings of the Berekhat Ram figure (Fig. 18) are again in serious error. The authors illustrate the supposed 'left' side of the figurine (Fig. 18b) as containing an 'arm.' That side, however, does not contain an arm, as indeed I had remarked, 'There is no arm on the left side. A study of the surface reveals that it contains intrusions ... which would have made scraping and carving difficult.' I had also written that, pragmatically, there was not enough tuffic material or mass on the left side from which to carve or incise an arm (Marshack 1997a, 330). Not only have the authors reversed the right and left sides but their profile rendition of the 'left' side has an 'arm' supposedly described by Marshack. It is the right side that, in fact, has that arm (Marshack 1997a, $328,331)$. Their illustration also suggests that I indicated a vertical groove on the left side (their mistaken right side), apparently suggesting according to Marshack a schematic second 'arm'. Such an 'arm' was never suggested or described in the original paper. The authors also illustrate two vertical grooves on the rear as supposedly described by Marshack, apparently representing the back of two arms. Those vertical grooves do not exist and were not men- tioned or described (Marshack 1997a). A comparison of their schematic illustrations (Fig. 18a) with the text and colour photos in the original paper suggests that the authors had not studied the figurine as a 'figurine' but had primarily made close-up scanning electron microscopic studies and photos at particular points. Significantly, they also incorrectly illustrate my description of the 'arm' on the right side. I had indicated and described an arm that was bent at the elbow; they depict my analysis as a presumably unbent, straight, hanging arm. Their illustration of what 'Marshack' asserted and claimed is not, therefore, what 'Marshack' had documented or written. Of particular importance, however, is the fact that the authors could reverse the right and left sides of the figurine, apparently indicating that their study of the artefact had been primarily conducted at high magnification. It may not be too strong to suggest that the authors did not perceive or study the figurine as a possible figurine but primarily as a series of areas that could be subjected to validation or invalidation at high magnification. The Berekhat Ram figurine is, however, far more than its 'validated' parts. The odd nature of the authors' errors puts into question the subsequent interpretations and discussion offered by d'Errico \& Nowell, in much the way that d'Errico's earlier errors in both illustrating and referring to the Rochedane artefact (Ro48) put into question his exhaustively careful, precisely analytical, strongly assertive, and subsequent logical arguments for the non-existence of 'notation' (cf. Marshack 1995c; 1996a, figs. 1-3).

Excellent technology and experimentation will aid a scientific inquiry and provide a certain level of data, but these are not in and of themselves, and therefore, adequate science. Technology and experimentation, particularly in the extraordinarily difficult realm of investigating the cognitive and perceptual processes that are involved in human symbolling, should be utilized with at least some knowledge of, or preparation for, investigating the nature and complexity of the symbolling and problem-solving processes being investigated and tested. Save for a careful use of microscopy and SEM on selected areas of the figurine, and for conducting tuffic experiments, d'Errico \& Nowell had apparently not prepared themselves for a central problem in the study of early symbolling, 'the modification of natural forms', and they had apparently neither carefully studied the figurine nor carefully read the original paper.

D'Errico \& Nowell's article is, nevertheless, of major importance. It marks a significant stage or 
step in the study and evaluation of the early human capacity. The technologies developed and utilized by d'Errico are significant contributions, in large measure because they represent the development of a technology. These, and the many studies conducted by d'Errico and his colleagues (cf. d'Errico et al. 1998c), have opened the investigation of early human symbolling to widening acceptance. Of still greater importance, they have opened it to other and deeper levels and modes of methodological, systematic inquiry. In this comment and in my earlier research, I have touched on a few of these directions. It must be recognized that the truly relevant evolutionary questions cannot be addressed within the limitations of, or in the manner of, the approach adopted by the authors.

I have argued in many papers for a diverse range of problem-solving and symbolling capacities among archaic humans, a range from which selection probably occurred for enhancement within the Neanderthals and anatomically moderns. That suggestion offers an evolutionary model that is different from those which are dependent on morphology, DNA, and tool types, forms of quantifiable data that do not and cannot measure inherent or potential capacity. Anatomically modern humans and the Neanderthals, for instance, both lived in the Near East during the Middle Palaeolithic and both used a Middle Palaeolithic Levalloisian technology. Both probably had language and, as I have indicated, modes of symbolling, but as I (Marshack 1996b) and others have suggested, there had begun in that period to be significant cultural changes among these human groups. The nature of, and the differences in, a shared and derived human capacity, and the reasons for, and the relevance of, the cultural changes that each began to initiate, demands theoretical and analytical investigation - but at levels beyond that offered by quantifiable differences in morphology, tools, DNA, or a particular artefact.

\section{Notes}

1. Anyone who has worked with the incised bone and stone materials of the Upper Palaeolithic and has carefully removed soil and sand from the declivities, often revealing an 'unweathered' surface and even the presence of unseen red ochre in an engraving, would understand the process.

2. I note, parenthetically, that as the analysis of the Berekhat Ram figurine proceeeded it could increasingly be seen as a process that had utilized and produced categorical contours and forms. In notational studies, by contrast, analysis requires a determination of the nature and structure of a sequence of positional sets and subsets, representing an entirely different type of visual and cognitive problem-solving both in the original production and in the subsequent analysis. The uses of microscopy in the study of such varying modes, though they may seem to be similar, are profoundly different.

\section{Reply from Francesco d'Errico \& April Nowell}

We are grateful to our colleagues for their insightful and thought-provoking comments. In the remarks that follow we shall attempt to address three main issues raised by their comments: a) the anthropogenic origin of the Berekhat Ram object; $b$ ) possible alternative interpretations (functional, non-purposive, symbolic); and c) the possible representational nature of the object. Finally, we will address the implications of our findings for the understanding of the evolution of symbolic behaviour.

The primary aim of our article was to investigate the anthropogenic origin of the Berekhat Ram object. We are pleased to see that all of the commentators seem to agree that, minimally, a large number of the modifications to this object should be interpreted as the result of human action and not of natural agents. It is also interesting to note that, with the exception of Marshack, all of our colleagues seem to welcome this new study as well as the analytical and, to some extent, the theoretical framework in which this research was carried out. The implications of this consensus extend beyond this study to the larger realm of archaeological investigation. It may be that after many disparate attempts to verify the anthropogenic origins of possible non-utilitarian objects found in Lower and Middle Palaeolithic contexts, we now have an agreed-upon method for doing so that stresses the need to make explicit the criteria by which objects are evaluated, and combines the use of actualistic, experimental, and contextual data. This approach is a more informed way of guiding archaeological reasoning. Recently, it has been successfully applied in other contexts (see for example d'Errico \& Villa 1997; d'Errico et al. 1998c; Chase \& Nowell 1998) and may become the accepted procedure for future research. Only Marshack seems to disagree with the view that the kind of study we present here is the necessary groundwork that must be completed before archaeologists can begin to discuss an object's significance.

As far as the more significant issue of the interpretation of the anthropogenic modifications documented by our analysis is concerned, we think that 
we differ from some of the commentators in what we believe to be the appropriate manner in which to address this question. What we consider to be a step by step discussion of related but separate issues (the possible symbolic value of the object, its representational nature and the subject represented) is treated by some of the commentators as one single topic. This means that an interpretation of the Berekhat Ram object becomes an 'all or nothing issue'. In other words, if they see no evidence that this object should be interpreted as a figurine then it follows for them that it is not symbolic. We argue that, while these issues build upon each other, they should be treated separately as each of them entails a different level of inference.

\section{Anthropogenic origin of the Berekhat Ram object}

Essentially, for Marshack it appears that we have become so involved in applying 'sophisticated technology' to a problem he has already 'solved' that in the process we lost sight of the 'important' issues namely the cognitive processes behind the Berekhat Ram object. There are three points arising from Marshack's present criticisms which we would like to discuss.

First, Marshack's main comment seems to be that his and our analyses of the object have been carried out at different levels, as the aim of his study was not to demonstrate the human origin of the modifications, but rather to test 'the nature of the perceptual, problem solving and cognitive processes that may have been involved' in the production and use of the object. This, however, was not the objective of his article in Antiquity. In his introduction to that article, he clearly stated that his research had 'indicated that the pebble had been intentionally modified' (Marshack 1997a, 328). He then devoted approximately 7.5 pages to describing these modifications and only 1.5 pages to the possible implications for cognition. Furthermore, it is difficult for us to imagine how an editor of an internationally known journal would accept a paper that focused on what the implications of a piece of stone might be if the stone was not a stone but a carving.

Second, neither in earlier publications in which he discusses the object, nor in his present comment, does Marshack provide any clues to help us understand what criteria he has used to test his assumptions concerning the possible cognitive process involved in the manufacture of the Berekhat Ram object. We do not see how an archaeological inquiry aimed at discussing the cognitive implications of an object could ignore the identification or use of methods to first establish whether the object is or is not human made and, if anthropogenic, how (i.e. with what techniques, tools, motion, succession of actions, timing etc.) the anthropogenic modifications were produced. These sequences of actions are in fact sequences of choices and decisions which may reflect the cognitive processes at work. Thus, they will be integral to any argument Marshack wishes to make concerning the nature of these processes. How, for example, could Marshack evaluate the cognitive implications of the use of such a tiny fragment of volcanic rock, or of the choice of a piece with this particular morphology, if he had no idea of the size and morphological variability of the available raw material at the site? How could he recognize 'microscopic evidence of intentionality without a need for experimentation' if he didn't know whether similar grooves occurred naturally on this type of material? How can he think that he is in a better position than us to judge what a ground surface would look like after $250 \mathrm{Kr}$ if, unlike us, he has no idea of what a surface which had been ground would look like when it was made? How can he seriously suggest that we are wrong in documenting the modifications on the object one by one, rather than deciding from the beginning, as he has, that it is a figurine? We therefore reject as epistemological nonsense Marshack's idea that we, and he, have carried out two different or, at best, complementary studies - one devoted to the 'interpretation' and the other to the 'validation' of the object. The two, in our view, go inevitably together, as an 'interpretation' without method is no more than a personal opinion. In other words, and contrary to Marshack beliefs, we have never argued that the 'intent must remain a black box'. The criticisms levelled at our interpretation of the Berekhat Ram object by several commentators clearly demonstrate this. We simply believe that before one can open that box and share its content with the scientific community, one has first to secure a reliable framework of inferences that enable a link to be established between observation and interpretation.

We apologize for any errors we might have made in reporting Marshack's description of the object. We were, however, aware of this possibility and for this reason clearly stated in our introduction that we were reporting Marshack's results 'as we understand them' and that 'teasing apart the natural features of the figurine from those considered by Marshack as intentionally modified is a difficult task as he blends them together in his description'. Con- 
trary to Marshack, however, we do not see how this may weaken our argument. We have tried to show that, independently of its depictive nature, this object may be relevant for the debate about the origin of symbolism because of the modifications it exhibits, not that it should be interpreted as a figurine. The lack of an arm, in this respect, seems more to jeopardise Marshack's hypothesis than our own.

Finally, one of us (d'Errico 1989; 1992; 1995b; $1996 a, b)$ has already discussed in this journal and elsewhere the various problems concerning Marshack's work on Palaeolithic 'notations'. In particular, and contrary to his claim, d'Errico has never stated that there was no notation in the Upper Palaeolithic, but that the theoretical and analytical grounds on which Marshack and others have based their identification of 'notations', were, at best, debatable. It is precisely by developing a novel theoretical reflection and identifying pertinent analytical criteria that d'Errico (d'Errico 1995; 1998; in press; d'Errico \& Cacho 1994) was eventually able to show that, in some cases, objects previously considered as notations by Marshack and others should not be interpreted as such and that, in other cases, they should be interpreted as notations, but of a kind different from that suggested by Marshack. The notational character of Ro48 is discussed in d'Errico 1996c. We reiterate all this here because we believe that, as with the Berekhat Ram object, it is precisely the clarification of methods and the reanalysis of the archaeological evidence, encorporating, as Bar-Yosef suggests, certain basic standards of the Natural Sciences, that has transformed the topic of notation from a situation where archaeologists are forced to rely on one person's opinion into an area of proper scientific inquiry and debate.

\section{Alternative hypotheses}

\section{A functional hypothesis}

We fully concur with Close, Zilhão, Mithen and Wynn that a functional or 'non-purposive' interpretation is not 'refuted' by our analysis. What 'refutation' means, however, in the context of this research needs to be discussed and we take the opportunity of this reply to delve deeper into this matter. In theory, refuting alternative interpretations to a symbolic explanation would be relatively easy. It would be sufficient, following a traditional archaeological practice, to demonstrate that this object shares technological, stylistic and dimensional features with roughly contemporaneous carvings found at similar sites. This would suggest that the Berekhat Ram ob- ject is an example of a known archaeological 'type'. Furthermore, because it was found in a specific context we might also be able to demonstrate coherent morphological and technical variations in time and space that we would then interpret as evidence of an evolutionary trend of a given technocomplex. We would then compare these results with those coming from an analysis of the raw material and the techniques used at this site and in neighbouring areas to produce colorants. This might show that, though made from similar raw material, this object is the result of a reduction sequence that differs from the kind used to produce colorants but is similar to the techniques used to carve this specific category of symbolic objects. We might also be able to demonstrate this through the experimental replication of both techniques and through a study of the morphological variability of contemporary carvings.

Unfortunately, this is impossible to do for the Berekhat Ram object, as the Acheulean record of the Near East lacks evidence of both carvings and the use of colorants. It is difficult, therefore, to apply the otherwise logical refutational procedure that Zilhão, for example, suggests. We may, however, rely on what we know about the techniques used to process colorants in the Lower and Middle Palaeolithic, as well as the variability in the modification and use of colorants in more recent times, to assess the significance of this object. Such an approach suggests that the anthropogenic modifications documented by our analysis of the artefact contradict a functional interpretation. In other words, while our analysis cannot logically refute a functional interpretation, we would argue that there are enough inconsistencies in this interpretation to warrant the further investigation of this matter.

A large number of red, yellow and black colorants are known from Lower and, particularly, Middle Palaeolithic sites in Europe (Bordes 1952; Wreschner 1982; Chase \& Dibble 1987; Demars 1992). They increase in number significantly at some late Neanderthal sites such as the Grotte du Renne at Arcy where the Châtelperronian layers contain heavy concentrations of ochre. In fact, approximately $9 \mathrm{~kg}$ of ochre fragments have been recovered from these layers (Couraud 1980). In the Near East, pieces of red and yellow ochre, one showing clear traces of use, have been found in the Mousterian levels of Qafzeh, dated to c. 90-100 Kya, in apparent association with the burials of Anatomically Modern Humans (Vandermeersch 1969; Hovers et al. 1997). An increasing number of pieces of red ochre, often exhibiting traces of use, have been also been reported 
over the last few years from southern African MSA contexts (Singer \& Wymer 1982; Knight et al. 1995; Watt 1998a,b; Henshilwood pers. comm.). The use of colorants becomes ubiquitous in the Upper Palaeolithic as well as in Late Pleistocene and Holocene contexts all over the world (e.g. d'Errico \& Vilhena Vialou 1999). One of us (FD) has examined a large sample of these objects from Europe, America and Africa.

In spite of the diversity of chronological, geographical, cultural, and probably symbolic contexts in which these materials were processed and used, they show a limited repertoire of modifications, diagnostic of the different techniques and motions applied to them. These lumps are either scraped or ground. In the former case, the use of the lateral edge of a blank or of a retouched tool produces a palimpsest of sets of parallel shallow striations. This technique is found both on flat/slightly concave and rounded surfaces. The use of pointed tools results in surfaces covered by individual straight subparallel or intersecting grooves. The presence of deep grooves and of changes in the direction of the grooves when crossing or joining an already existing groove are among the diagnostic features of this technique, which is almost exclusively used on flat or slightly concave surfaces.

Grinding produces one or more isolated or adjacent facets covered by fusiform striations - the width of which depends on the size and hardness of the abrasive grains contained in or present on the surface of the grinding tool. The anthropogenic origin of other reported functional modifications is uncertain. We suspect, for example, that deep ogival concavities on pieces of ochre from southern African MSA contexts like Klasies Shelter (Watt 1998b), interpreted as the product of drilling, may instead be the result of perforations made by marine molluscs. This is certainly the case for all the pieces from the MSA levels of Blombos cave that otherwise exhibit genuine traces of scraping (d'Errico \& Henshilwood unpublished data).

Although examples of the grooving technique may, in a few instances, be found in different places on these objects, there is no example, to our knowledge, of a modified lump of colorant where this technique was used to produce either a single groove encircling the object or a 'U'-shaped groove. Similarly, we have never seen, among the pieces examined so far, a piece of colorant where the ground surface forms an acute angle with a more prominent area that is unmodified, as is the case for the groove between the 'chest' and the 'head' of our object. Pieces of ochre that have been completely shaped by grind- ing and/or scraping, are known in MSA, Mousterian, and Upper Palaeolithic as well as more recent contexts (Fig. 24, top). These lumps of colorant, however, are different from the object under study here as they generally exhibit one or more pointed ends or thin rounded edges and diagnostic use-wear. These features are typical of objects that have been used as colorants to apply colour accurately on a chosen surface. Considering what has been summarized above concerning the object's morphology and texture, colorant powder would have been more quickly and effectively produced by grinding one face of this object against a basalt flake or smashing it with a similar tool.

In sum, there is little doubt that the difference between the types of modifications observed on Palaeolithic pieces of ochre used for functional purposes, and those recorded on the Berekhat Ram object, call into question a purely functional hypothesis. It is obviously difficult to know what such a range of modifications may have meant in an Acheulean context. If we use more recent societies for comparison, however, it is a fact that non-functional modification of pieces of ochre as in the production of personal ornaments (Fig. 25, cf. also Magdalenian beads made of ochre from Belvis and Gandil shelters, southern France), carvings (cf. Gravettian female figurine in haematite from Petrkovice), engravings (cf. Magdalenian representations of horses on ochre slabs from Lumentka and Urtiaga, Basque country) or in the decoration of 'functional' crayons (Fig. 24, centre and bottom) are systematically associated with fully symbolic societies in which these objects represent one aspect of complex systems of representation. It is probably relevant, for the question that occupies us here, that a number of objects of this type appear in MSA contexts well before the MiddleUpper Palaeolithic transition in Europe (Watt $1988 a, b)$. When they occur, these objects are generally small, even smaller than our specimen. Furthermore, sequences of notches, or notches producing grooves encircling the object to facilitate suspension, or the production of symbolic depictions, are not rare.

Whether this is the case for the society which produced our object is difficult to say, and we agree in this respect with Bar-Yosef that there is no obvious model to use as an analogy for early hominid behaviour. What is certain, however, is that this object cannot be accounted for by attributing it to the production of ochre. Finally, it is important to note that the Berekhat Ram object does not need to have served either a utilitarian function or a symbolic func- 
tion but could be a product of both, as is clear with more recent objects made of similar materials.

\section{Non-purposeful behaviour}

Archaeologists have often been accused of interpreting all evidence they are otherwise unable to explain, as ritual. We wonder whether such criticism should not be extended to the non-purposive or random 'doodling' interpretation of material culture. To test the nonpurposive hypothesis one should first be able to distinguish clearly what may or may not be purposeful in human agency. Subsequently we need to find a general pattern that is valid for our genus, and identify clues that will enable us to distinguish purposive from non-purposive behaviours in the archaeological record. Our commentators seem to follow a different path. For some of them it is the uniqueness and the idiosyncratic character of the object that is key to a nonpurposive interpretation. In contrast, Wynn believes that a large number of our actions, like that of randomly drawing while on the telephone, are nonpurposive and that this object might just be another example of such behaviour. The former position must explain why, in an archaeological interpretation, uniqueness should be taken as synonymous with nonpurposive action, while the latter must explain why, if nonpurposive actions are so common, we do not find many instances of them in the archaeological record. We challenged the non-purposive interpretation from a different perspec-
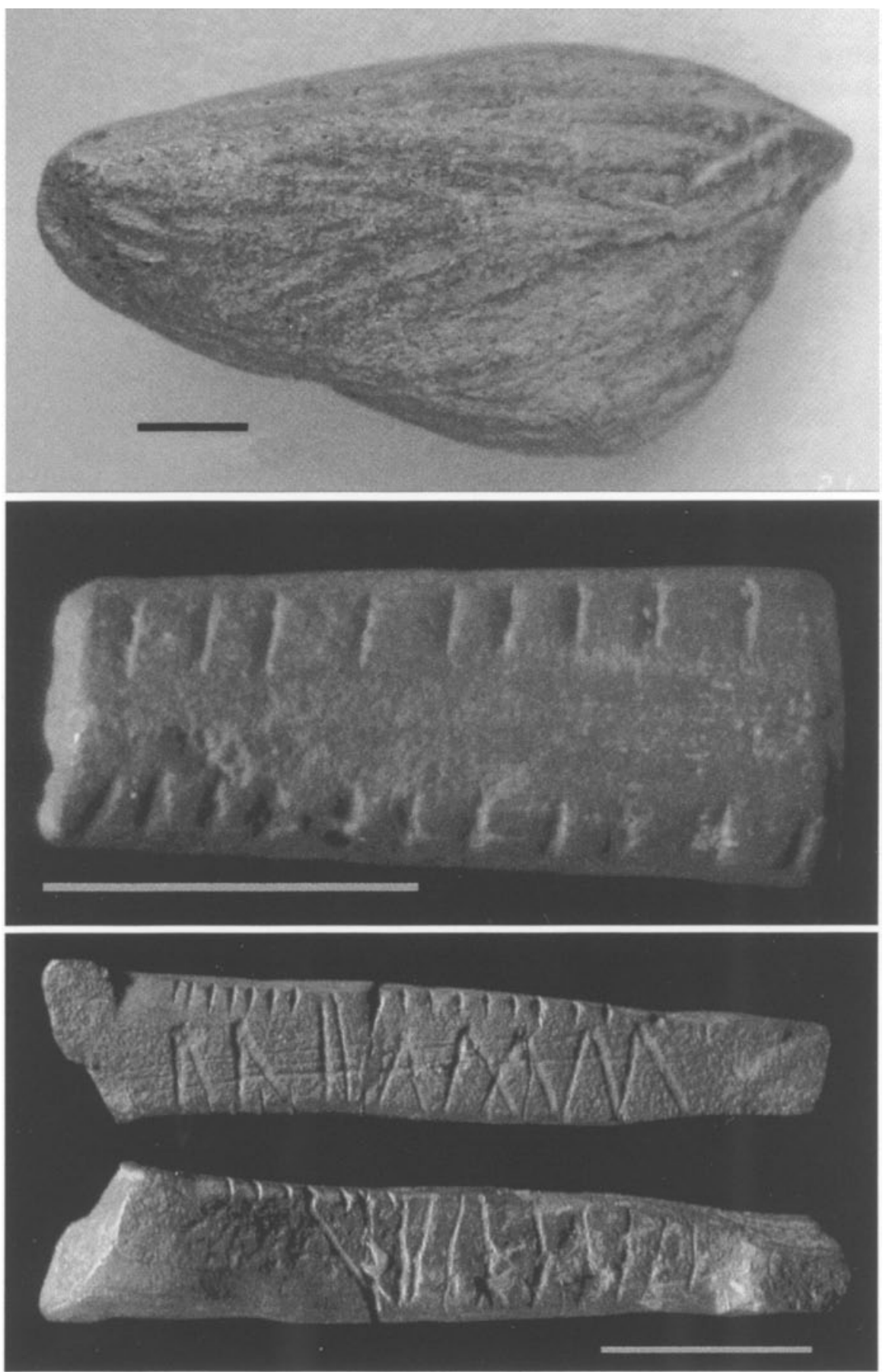

Figure 24. Top: crayon of haematite found at Lascaux during Glory excavations (courtesy of M. Menu). Centre: ochre pencil decorated with four sets of notches from the Abri Blanchard (Musée de l'Homme collection, probably Gravettian, photo F. d'Errico). Bottom: Late Magdalenian decorated ochre pencil from Arancou shelter (courtesy of A. Roussot). Scale $=1 \mathrm{~cm}$.

tive. We argued that our experimental results suggest that the modifications observed on the object

appear to be the result of a deliberate motion possibly the reflection of a conscious project. 


\section{Representational nature of the Berekhat Ram artefact}

We have never argued that the Berekhat Ram object is a figurine. It is true, however, that we used that term as a convenient way of referring to the object because that is how it is known in the literature much as the term 'scraper' is ubiquitous in discussions of lithic industries even though everyone suspects that these tools were most likely used for a variety of functions and not merely scraping. Nonetheless, as Mithen has pointed out, it is an unfortunate choice of terms because of its connotation. Even Mithen himself, however, slips into referring to the object as 'the Berekhat Ram figurine' in his conclusion for, we suspect, the same reason we did throughout our article. Not only have we never argued that the object is a figurine, but in our article we emphasized that 'the recognition of a human body in this specimen is problematic. It ultimately depends on the criteria one employs to identify a human figure in a carved object and the level of uncertainty that one is willing to accept as part of this identification'. In other words, unlike Marshack who is able to identify 'managed hair', we are hesitant to ascribe female characteristics to this object because of the difficulty in defining consistent criteria for doing so and the lack of comparable material. We have suggested that the subject of the representation is less important than the fact that at least some of the modifications

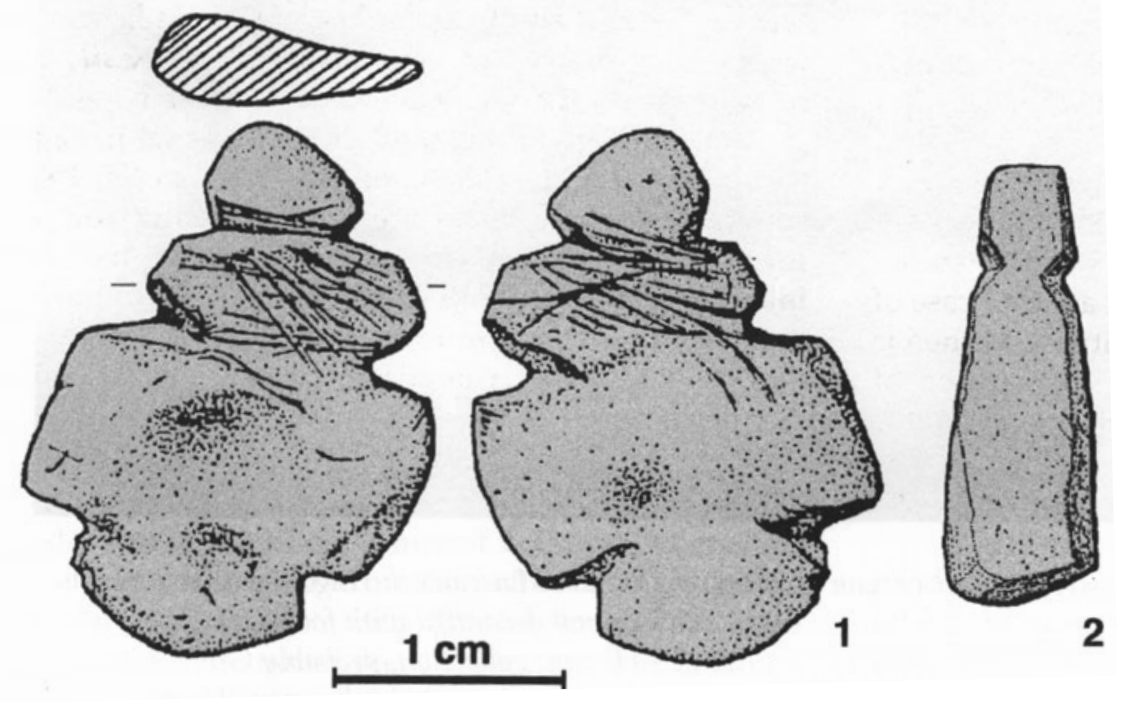

Figure 25. Pendants in haematite from the ceramic levels of the rock art site of Ferraz Egreja (Mato Grosso), c. AD 1000. 1 is interpreted as a possible anthropomorphic representation (1 modified after Vialou \& Vialou 1994, n. 2 redrawn from Vialou et al. 1996). are inconsistent with a purely functional (and probably non-purposeful) explanation.

Wynn suggests that we have unwittingly created a paradox by arguing that if the Berekhat Ram artefact is depictive then it is not iconic and, therefore, must be symbolic. Our argument is not as simplistic as Wynn would have it. What we wrote was that 'if iconic, the representation is far enough away from the subject for which it stood to suggest' that a closer resemblance was not needed to establish a meaningful link between the two'. Conversely, if the object is symbolic, 'we are forced to admit that the arbitrary link between the meaning and its material appearance was solid enough' to be established through a visual rather than an encrypted representation of the symbol. But we will address Wynn's paradox nonetheless. He says that if we agree that when the relationship between a symbolic artefact and its referent is not obvious it must be created in the mind of the observer then we must, by extension, agree that if an object is iconic it is not symbolic. He uses the Vogelherd horse as an example. It should be obvious, however, that while an object can be symbolic without being iconic (in fact that is part of the definition of a symbol) an object can be symbolic as well as being iconic. Consider, for example, an Eiffel Tower key chain available in any souvenir shop in Paris. It is at once an icon of the actual Eiffel Tower in Paris as well as a symbol of the entire city of Paris and/or all of France.

We would like to make one further point concerning Wynn's comments. We believe that Wynn answers his own question (and that of Zilhão) about the unusual combination of features (small size and porous raw material) which characterize the Berekhat Ram object. As we lack theoreticallygrounded hypotheses to grasp what pre-modern depiction might have looked like, it is difficult to reject the symbolic meaning or the depictive nature of such an ancient object by relying on modern carving technology to guide our interpretation. Moreover, ethnographic and archaeological examples of small objects carved on porous material can be found. Thus material and size do not appear to be obstacles to symbolic interpretations. 


\section{Models for the origin of symbolling}

Finally, we would like to address comments concerning the origins of symbolling. Contra Mithen, we believe that making a distinction between a biological and a historical / cognitive model for the origin of symbolling is a logical way to initiate a discussion of the mechanisms which may be responsible for the emergence of the capacity for symbolling. Over the last few years, much of the debate which has taken place on the pages of prestigious international journals (e.g. Appenzeller 1998) concerns whether or not a speciation event is required to explain the emergence of new cognitive abilities including symbolling. Many researchers still adhere to the belief that symbolling is a 'modern' behaviour which, by definition, is characteristic only of anatomically modern humans.

Plastic and remouldable as neural networks may be, potentially immense changes in culture and biology during an organism's life-span alone cannot account for the cognitive changes we are discussing here. There are two facts which are relevant to this discussion. First, chimpanzees in the wild have never been seen producing symbolic material culture. Second, and apparently in contradiction to Mithen's views, we have consistent archaeological evidence that confirms that Neanderthals produced and used symbolic objects (d'Errico et al. 1998a,c; Zilhão \& $\mathrm{d}^{\prime}$ Errico 1999). This is demonstrated by the presence of a varied repertoire of personal ornaments, decorated bone tools and, in one instance, the byproducts of their manufacture, in late Neanderthal contexts. These bone tools and ornaments are different from those produced by contemporary modern humans and they show extensive traces of wear indicating they have been used. In light of this evidence it becomes difficult to assume, as Mithen does, that Neanderthals made the ornaments without knowing what to do with them. Is this yet another case of non-purposeful behaviour? Why is it that Mithen is willing to attribute much of the emergence of symbolling to the plasticity of the human brain yet is at the same time so reluctant to concede any of this ability to Neanderthals? We do not understand the basis upon which Mithen consistently ascribes an inherent, and thus biological, cognitive inferiority to Neanderthals if it is not due to the need to 'discard evidence when it does not fit with' his rather inflexible model of human cognitive evolution. It may be that modern human and Neanderthal interaction, behaviour and cultural evolution are far more complex topics than Mithen perceives them to be.
In our view, it is clear that we face a phenomenon in which biology and culture interacted. The central questions concern how and when and among what hominid group(s) symbolism emerged and what traces of this phenomenon are recoverable from the archaeological record. In this respect, we welcome the distinction Mithen makes between the possible symbolic nature of non-utilitarian artefacts and the cognitive mechanisms that may have governed their production and use. This distinction may provide an explanation for the unexpected pattern that faces archaeologists studying the nature and the temporal and geographic distribution of these artefacts. This pattern demonstrates the need to develop, as Wynn terms them, theoretically-grounded-hypotheses, to study the initial stages of symbol use. We do not understand, however, how Mithen can simultaneously advocate this approach and accept Gamble's light-switch analogy. This analogy is paradigmatic of the opposite stance - the use of modern symbols as a unique referential to identify past symbolic societies.

\section{Conclusion}

In sum, the results of our study support an anthropogenic origin for the Berekhat Ram object. We have argued that many of the modifications documented on the object are inconsistent with a purely functional (and possibly non-purposive) explanation. We further emphasized that it may not be possible to identify what, if anything, the object may depict and we are certainly not arguing that it is necessarily a figurine. We believe, however, that its possible subject matter is less important than the fact that it may have had a symbolic function. As Close notes, the results of our study make the Berekhat Ram object a mystery that will not soon disappear. We find it interesting that while all of the commentators commend us for our rigorous methodology and for explicitly stating the criteria by which we evaluated this object, the majority of them do not seem to accept the possible implications of these results and, at the end of it all, have retained the same interpretation of the find they held before reading this article. We feel sure that this is an issue that will continue to be debated for some time to come.

\section{Acknowledgements}

We thank Alain Roussot and Michel Menu for allowing us to use the photos at the top and at the bottom of Figure 24 . 


\section{References}

Adams, K., 1951. Der Waldelefant von Lehringen, ein Jagdbeute des Diluvialen Menschen. Quartar 5, 7992.

Anderson-Gerfaud, P., 1990. Aspects of behaviour in the Middle Palaeolithic: functional analysis of stone tools from southwest France, in The Emergence of Modern Humans: an Archaeological Perspective, ed. P. Mellars. Edinburgh: Edinburgh University Press, 389-418.

Appenzeller, T., 1998. Art: evolution or revolution? Science 282, 1451-4.

Bahn, P., 1996. New developments in Pleistocene art. Evolutionary Anthropology 4, 204-15.

Bahn, P. \& J. Vertut, 1997. Journey through the Ice Age. London: Weidenfeld \& Nicolson.

Bar-Yosef, O., 1992. The role of western Asia in Modern Human origins. Philosophical Transactions of the Royal Society of London B 337, 193-200.

Bar-Yosef, O. \& N. Goren-Inbar, 1993. The lithic assemblages of the site of Ubeidiya, Jordan Valley. Qedem $34,1-266$.

Bednarik, R.G., 1992. Palaeoart and archaeological myths. Cambridge Archaeological Journal 2(1), 27-57.

Bednarik, R.G., 1994. Art origins. Anthropos 89, 169-80.

Bednarik, R.G., 1995. Concept-mediated marking in the Lower Palaeolithic. Current Anthropology 36, 605-16.

Bednarik, R.G., 1997. The role of Pleistocene beads in documenting hominid cognition. Rock Art Research 14(1), 27-41.

Belitzky, S., N. Goren-Inbar \& E. Werker, 1991. A Middle Pleistocene wooden plank with man-made polish. Journal of Human Evolution 20, 349-53.

Bordes, F., 1952. Sur l'usage probable de la peinture corporelle dans certaines tribus moustériennes. Bulletin de la Société Préhistorique Française 49, 169-70.

Bosinsky, G., 1991. The representation of female figures in the Rhineland Magdalenian. Proceedings of Prehistoric Society 57, 51-64.

Bosinsky, G. \& F. d'Errico, in prep. Les représentations féminines gravées de Gönnersdorf. (Der MagdalénienFundplatz Gönnersdorf, Band 6.) Wiesbaden: Franz Steiner Verlag GMBH.

Byers, M., 1994. Symboling and the Middle-Upper Palaeolithic transition. Current Anthropology 35(4), 369-99.

Byers, M., 1999. Communication and material culture: Pleistocene tools as action cues. Cambridge Archaeological Journal 9(1), 32-41.

Chase, P.G., 1990. Sifflets du Paléolithique Moyen (?). Les implications d'un coprolithe de coyote actuel. Bulletin de la Société Préhistorique Française 87, 165-7.

Chase, P.G., in press. Integrating Palaeolithic archaeology into the study of the evolution of human intelligence, in In the Mind's Eye, ed. A. Nowell. Ann Arbor (MI): International Monographs in Prehistory.

Chase, P.G. \& H.L. Dibble, 1987. Middle Palaeolithic: a review of current evidence and interpretations. Journal of Anthropological Archaeology 6, 263-96.
Chase, P.G. \& H.L. Dibble, 1992. Scientific archaeology and the origins of symbolism: a reply to Bednarik. Cambridge Archaeological Journal 2(1), 43-51.

Chase, P.G. \& A. Nowell, 1998. Taphonomy of a suggested Middle Paleolithic bone flute from Slovenia. Current Anthropology 39(4), 185-94.

Couraud, C., 1980. Les colorants de la grotte du Renne (Arcy-sur-Cure, Yonne). Bulletin de la Société Préhistorique Française 77(6), 162.

Crémades, M., H. Laville, N. Sirakov \& J.K. Kozlowski, 1995. Une pierre gravéede 50,000 ans вр dans les Balkans. Paléo 7, 201-9.

Davidson, I., 1990. Bilzingsleben and early marking. Rock Art Research 7, 52-6.

Davidson, I. \& W. Noble, 1989. The archaeology of perception. Current Anthropology 30, 125-55.

Davidson, I. \& W. Noble, 1993. Tools and language in human evolution, in Gibson \& Ingold (eds.), 363-88.

Deacon, T., 1992. Brain-language co-evolution, in The Evolution of Human Languages, eds. J. Hawkins \& M. Gel-Man. Redwood City (CA): Addison-Wesley, 4983.

Demars, P-Y. 1992. Les colorants dans le Moustérien du Périgord. L'apport des fouilles de F. Bordes. Bulletin de la Société Préhistorique de l'Ariège 47, 185-94.

d'Errico, F., 1989. Reply to Alexander Marshack (1989). Wishful Thinking and Lunar 'Calendars'. Current Anthropology 30(4), 494-500.

d'Errico, F., 1991. Carnivore traces or Mousterian skiffle? Rock Art Research 8, 61-3.

d'Errico, F., 1992. Reply to A. Marshack. Rock Art Research $8(3), 122-30$.

d'Errico, F., 1995a. L'art gravé azilien. De la technique à la signification. (XXXIe Supplément à Gallia Préhistoire.) Paris: CNRS.

d'Errico, F., 1995b. New model and its implications for the origin of writing: La Marche antler revisited. Cambridge Archaeological Journal 5(1), 3-46.

d'Errico, F., 1996a. Marshack's approach: poor technology, biased science. Cambridge Archaeological Journal 6(2), 39-45.

d'Errico, F., 1996b. Comment to Elkins: on the impossibility of close reading. The case of Alexander Marshack. Current Anthropology 37(2), 207-8.

d'Errico, F., 1996c. L'art azilien dans son contexte européen, in Art in the Palaeolithic and Mesolithic, eds. A. Beltran \& A. Vigliardi. (XIII Int. Congress of Prehistoric and Protostoric Sciences.) Forlí: Abaco Edizioni, 11-20.

d'Errico, F., 1998. Palaeolithic origins of artificial memory systems: an evolutionary perspective, in Cognition and Material Culture: the Archaeology of Symbolic Storage, eds. C. Renfrew \& C. Scarre. (McDonald Institute Monographs.) Cambridge: McDonald Institute for Archaeological Research, 19-50.

d'Errico, F., in press. Memories out of mind: the archaeology of the oldest memory systems, in In the Mind's Eye: Multidisciplinary Perspectives on the Evolution of Human Intelligence, ed. A. Nowell. Ann Arbor (MI): International Mongraphs in Prehistory. 
d'Errico, F. \& C. Cacho, 1994. Notation versus decoration in the Upper Palaeolithic: a case-study from Tossal de la Roca, Alicante, Spain. Journal of Archaeological Science 21, 185-200.

d'Errico, F. \& A. Vilhena Vialou, 1999. Reduction sequences of colorant materials at the rock art of Santa Elina (Mato Grosso, Brazil), in New Approaches to Rock Art studies, Proceedings of the Conference 'News 95: International Rock Art Conference' Turin, September 1995, on CD-rom.

d'Errico, F. \& P. Villa, 1997. Holes and grooves: the contribution of microscopy and taphonomy to the problem of art origins. Journal of Human Evolution 33, $1-31$.

d'Errico, F., D. Baffier \& M Julien, 1998a. Les inventions des derniers Néanderthaliens. Pour la Science 254 80-83.

d'Errico, F., P. Villa, A. Pinto \& R. Idarraga, 1998b. A Middle Paleolithic origin of music? Using cave bear bone accumulations to assess the Divje Babe I bone 'flute'. Antiquity 72, 65-79.

d'Errico, F., J. Zilhâo, M. Julien, D. Baffier \& J. Pelegrin, 1998c. Neanderthal acculturation in western Europe?: a critical review of the evidence and its interpretation Current Anthropology 39 Supplement, S1-S44.

d'Errico, F., P. Villa, A. Pinto \& R. Idarraga, in press. La 'flüte' de Divje Babe et les accumulations naturelles d'ossements d'ours des cavernes, in Economie Préhistorique: Les Comportements de Subsistance au Paléolithique. Actes des XVIIIe Rencontres Internationales d'Archéologie et d'Histoire, eds. J-Ph. Brugal, L. Meignen \& M. Patou-Methis. Sophia Antipolis: Editions APDCA, 85-104.

Duff, A.I., G.A. Clark \& T.J. Chadderton, 1992. Symbolism in the Early Palaeolithic. Cambridge Archaeological Journal 2(2), 211-29.

Duhard, J.P., 1993a. Upper Palaeolithic figures as a reflection of human morphology and social organization. Antiquity 67, 83-91.

Duhard, J.P., 1993b. Réalisme de l'Image Féminine Paléolithique. (Cahiers du Quatérnaire 19.) Paris: CNRS.

Feraud, G., D. York, C.M. Hall, N. Goren-Inbar \& H.P. Schwarcz, 1983. 40 ar/39 ar age limit for an Acheulean site in Israel. Nature 304, 263-5.

Fullagar, R.L.K., D.M. Price \& L.M. Head, 1996. Early human occupation of northern Australia: archaeology and thermoluminescence dating of Jinmium rock-shelter, Northern Territory. Antiquity 70, 75173.

Gamble, C., 1982. Interaction and alliance in Palaeolithic society. Man 17, 92-107.

Gero, J. \& J. Mazzullo, 1984. Analysis of artifact shape using Fourier series in closed form. Journal of Field Archeology 11, 315-22.

Gibson K. \& T. Ingold (eds.), 1993. Tools, Language, and Cognition in Human Evolution. Cambridge: Cambridge University Press.
Gifford-Gonzalez, D.P., D.B. Damrosch, D.R. Damrosch, J. Pryor \& R.L. Thunen, 1985. The third dimension in ste structure: an experiment in trampling and vertical dispersal. American Antiquity 50, 803-18.

Gilman, A., 1984. Explaining the Upper Palaeolithic revolution, in Marxist Perspectives in Archaeology, ed. M. Spriggs. Cambridge: Cambridge University Press, 115-26.

Gimbutas, M., 1989. The Language of the Goddess. San Francisco (CA): Harper \& Row.

Goldberg, P., 1987. Soils, sediments and Acheulean artifacts at Berekhat Ram, Golan Heights, in Micromorphologie Des Sols-Soil Micromorphology, eds. N. Federof, L.M. Bresson \& M.A. Courty. Paris: Plaisir, 583-9.

Goren-Inbar, N., 1985. The lithic assemblages of Berekhat Ram Acheulean site, Golan Heights. Paléorient 11, 728.

Goren-Inbar, N., 1986. A figurine from the Acheulean site of Berekhat Ram. Mitekufat Haeven 19, 7-12.

Goren-Inbar, N. (ed.), 1990. Quneitra: a Mousterian site on the Golan Heights. Qedem 31, ??-??.

Goren-Inbar, N. \& S. Peltz, 1995. Additional remarks on the Berekhat Ram figurine. Rock Art Research 12, 353-4.

Goren-Inbar, N., I. Permann \& A. Heimann, 1986. Chemical mapping of basalt flows at Palaeolithic sites. Archaeometry 28, 89-99.

Gvozdover, M, 1995. Art of the Mammoth Hunters: Finds from Avdeevo. (Oxbow Monographs 49.) Exeter: Short Run Press.

Hayden, B., 1993. The cultural capacities of Neanderthals: a review and re-evaluation. Journal Human Evolution 24, 113-46.

Henshilwood, C. \& J. Sealy, 1997. Bone artifacts from the Middle Stone Age at Blombos Cave, Southern Cape, South Africa. Current Anthropology 38(5), 890-95.

Hovers, E., B. Vandermeersch \& O. Bar-Yosef, 1997. A Middle Palaeolithic engraved artefact from Qafzeh Cave, Israel. Rock Art Research 14(2), 79-87.

Jelinek, J., 1975. Pictorial Encyclopedia of the Evolution of Man. London \& New York (NY): Hamlyn.

Keeley, L.H., 1980. Experimental Determination of Stone Tool Uses: a Microwear Analysis. Chicago (IL): University of Chicago Press.

Klein, R.G., 1996. The problem of Modern Human origins, in Origins of Anatomically Modern Humans, eds. M.H. Nitecki \& D.V. Nitecki. New York (NY): Plenum Press, 3-17.

Knight, C., C. Powers \& I. Watts, 1995. The human symbolic revolution: a Darwinian account. Cambridge Archaeological Journal 5(1), 75-114.

Lestrel, P.E., 1989. Method for analyzing complex twodimension forms: elliptical Fourier functions. American Journal of Human Biology 1, 149-64.

Lieberman, P., 1995. Manual versus speech motor control and the evolution of language. Behavioral and Brain Sciences 18, 197-8.

Lorblanchet, M., 1999. La Naissance de l'Art: Genèse de l'Art 
Préhistorique. Paris: Éditions France.

Marshack, A., 1976. Some implications of Palaeolithic symbolic evidence for the origin of language. Current Anthropology 17(2), 276-82.

Marshack, A., 1984. The ecology and brain of two-handed bipedalism: an analytic, cognitive and evolutionary assessment, in Animal Cognition, eds. H.L. Roitblat, T.G. Bever \& H.S. Terrace. Hillsdale (NJ): Erlbaum, 491-511.

Marshack, A., 1985. Hierarchical Evolution of the Human Capacity: the Paleolithic Evidence. (Fifty-fourth James Arthur Lecture on 'The Evolution of the Human Brain,' 1984.) New York (NY): American Museum of Natural History.

Marshack, A., 1988. The Neanderthals and the human capacity for symbolic thought: cognitive and problem-solving aspects of Mousterian symbol, in L'Homme de Neandertal, vol. 5, ed. O. Bar Yosef. Liège: ERAUL, 57-91

Marshack, A., 1989. Evolution of the human capacity: the symbolic evidence. Yearbook of Physical Anthropology 32,1-34.

Marshack, A., 1990. Early hominid symbol and evolution of the human capacity, in The Emergence of Modern Humans, ed. P. Mellars. Edinburgh: Edinburgh University Press, 457-98.

Marshack, A., 1991a. The female image: a 'time-factored' symbol: a study in style and modes of image use in the European Upper Palaeolithic. Proceedings of the Prehistoric Society 57(1),17-31.

Marshack, A., 1991b. A reply to Davidson on Mania \& Mania. Rock Art Research 8, 47-58.

Marshack, A., 1991c. The Roots of Civilization. 2nd edition. Revised and enlarged. Mt Kisco (NY): Moyer Bell Ltd.

Marshack, A., 1995a. A Middle Palaeolithic symbolic composition from the Golan Heights: the earliest known depictive image. Current Anthropology 37(2), 356-65.

Marshack, A., 1995b. Concerning the 'geological' explanation of the Berekhat Ram figurine. Current Anthropology 35, 495.

Marshack, A., 1995c. Methodology and the search for notation among engraved pebbles of the European late palaeolithic: a review of F. d'Errico, 1994, l' Art gravé azilien: de la technique à la signification. Gallia Préhistoire XXX. Antiquity 69(266), 1049-51.

Marshack, A., 1996a. La Marche revisited: good technology, poor science: a response to F. d'Errico. Cambridge Archaeological Journal 6(1),27-39.

Marshack, A., 1996b. A Middle Paleolithic symbolic composition from the Golan Heights: the earliest known depictive image. Current Anthropology 37(2), 357-65.

Marshack, A., 1997a. The Berekhat Ram figurine: a late Acheulean carving from the Middle East. Antiquity 71, 327-37.

Marshack, A., 1997b. Palaeolithic image making and symboling in Europe and the Middle East: a comparative view, in Beyond Art: Pleistocene Image and Symbol, eds. M. Conkey, O. Soffer, D. Stratmann \&
N.G. Jablonski. San Francisco (CA): California Academy of Sciences, 53-91.

Mellars, P., 1989. Major issues in the emergence of modern humans. Current Anthropology 30, 349-85.

Mellars, P., 1991. Cognitive changes and the emergence of modern humans in Europe. Cambridge Archaeological Journal 1(1), 63-76.

Mellars, P., 1996. The Neanderthal Legacy. Princeton (NJ): Princeton University Press.

Mithen, S., 1996a. The Prehistory of the Mind. London: Thames \& Hudson.

Mithen, S., 1996b. Comment on 'Concept mediate marking in the Lower Palaeolithic' by R. Bednarik. Current Anthropology 37, 666-70.

Mithen, S., 2000. Palaeoanthropological perspectives on the theory of mind, in Understanding Other Minds: Perspectives from Autism and Cognitive Neuroscience, eds. S. Baron-Cohen, H.T. Flusberg \& D. Cohen. Oxford: Oxford University Press, 488-502.

Mor, D., 1981. The volcanism in the Golan Heights. Geological Society of Israel Annual Meeting Katzrin, 30-32.

Morse, K., 1993. Shell beads from Mandu Mandu rockshelter, Cape Range Peninsula, western Australia, before 30,000 вP. Antiquity 67, 877-83.

Movius, H.L., 1950. A wooden spear of third interglacial age from Lower Saxony. Southwestern Journal of Anthropology 6, 139-42.

Noble, W. \& I. Davidson, 1996. Human Evolution, Language and Mind: a Psychological and Archaeological Inquiry. Cambridge: Cambridge University Press.

Oakley, K.P., P. Andrews, L.H. Keeley \& J. D. Clark, 1977. A reappraisal of the Clacton spear point. Proceedings of the Prehistoric Society 43, 1-12.

Olsen, S.L., 1996. Prehistoric adaptation to the Kazak steppes, in IUPPS 1996, Colloquium XXXI, The Evolution of Nomadic Herding Civilizations in the Northern European Steppes, 49-64.

Peirce, C.S., 1931-35. Collected Papers. Cambridge (MA): Hartshorne \& Weiss.

Pelcin, A., 1994. A geological explanation for the Berekhat Ram figurine. Current Anthropology 35, 674-5.

Povinelli, D., 1996. Chimpanzee theory of mind?: The long road to strong inference, in Theories of Theories of Mind, eds. P. Carruthers \& P.K. Smith. Cambridge: Cambridge University Press, 293-329.

Renfrew, C. \& P. Bahn, 1991. Archaeology: Theories, Methods, and Practice. London: Thames \& Hudson.

Renfrew C. \& E. Zubrow (eds.), 1994. The Ancient Mind. Cambridge: Cambridge University Press.

Rosenfeld, A., 1977. Profile figures: schematisation of the human figure in the Magdalenian culture of Europe, in Form in Indigenous Art, ed. P.J. Ucko. London: Duckworth, 90-109.

Schepartz, L., 1993. Language and modern human origins. Yearbook of Physical Anthropology 36, 91-126.

Schlanger, N., 1996. Understanding Levallois: lithic technology and cognitive archaeology. Cambridge Archaeological Journal 6(2), 231-54.

Shea, J., 1988. Spear points from the Middle Paleolithic of 
the Levant. Journal of Field Archaeology 15, 441-50.

Simek, J.F., 1992. Neanderthal cognition and the Middle to Upper Palaeolithic transition, in Continuity or Replacement: Controversy in Homo sapiens Evolution, eds. G. Brauer \& F.H. Smith. Rotterdam: Balkema, 23145.

Singer, R. \& J. Wymer, 1982. The Middle Stone Age at Klaiser River Mouth in South Africa. Chicago (IL): University of Chicago Press.

Soffer, O., J.M. Adovasio \& D.C. Hyland, forthcoming [2000]. Dressing the 'Venus', Upper Paleolithic iconography and perishable technologies. Current Anthropology 41(2).

Stepanchuk, V.N., 1993. Prolom II, a Middle Palaeolithic site in the eastern Crimea with non-utilitarian bone artifacts. Proceedings of the Prehistoric Society 59, 1737.

Stringer, C. \& C. Gamble, 1993. In Search of the Neanderthals: Solving the Puzzle of Human Origins. London: Thames \& Hudson.

Thieme, H., 1997. Lower Palaeolithic hunting spears from Germany. Nature 385, 769-71.

Tobias, P., 1998. Evidence for the early beginning of spoken language. Cambridge Archaeological Journal 8(1), $72-8$.

Tooby, J. \& I. DeVore, 1987. The reconstruction of hominid behavioral evolution through strategic modeling, in The Evolution of Human Behavior: Primate Models, ed. W.G. Kinzey. Albany (NY): State University of New York Press, 193-237.

Turk, I. (ed.), 1997. Mousterian Bone Flute and Other Finds From Divje Babe I Cave Site in Slovenia. Ljubljana: Opera Instituti Archaeologici Sloveniae.

Turk, I., J. Dirjec \& B. Kavur, 1995. Ali so v sloveniji nasli najstarejse glasbilo $\mathrm{v}$ europi? [The oldest musical instrument in Europe discovered in Slovenia?]. Razprave IV.razreda SAZU 36, 287-93.

Ucko, P.J., 1962. The interpretation of prehistoric anthropomorphic figurines. Journal of the Royal Anthropological Institute of Great Britain and Ireland 92, 38-54.

Vandermeersch, B., 1969. Découverte d'un object en ocre avec traces d'utilisation dans le Moustérien de Qafzeh (Israêl). Bulletin de la Société Préhistorique Française 69, 157-8.

Vialou, A. \& D. Vialou, 1994. Les premiers peuplements préhistoriques du Mato Grosso. Bulletin de la Société Préhistorique Française 91(4-5), 257-63.

Vialou, A., E. Badu, F. d'Errico \& D. Vialou, 1996. Les colorants rouges de l'habitat rupestre de Santa Elina, Mato Grosso Brazil. Techne 3, 91-7.
Villa, P. \& J. Courtin, 1983. The interpretation of stratified sites: a view from underground. Journal of Archaeological Science 10, 267-81.

Watt, I., 1998a. The origin of symbolic culture, in The Evolution of Culture, eds. R. Dunbar, C. Knight \& C. Power. Edinburgh: Edinburgh University Press, 11346.

Watt, I., 1998b. The Origin of Symbolic Culture: the Middle Stone Age of Southern Africa and Khoisan Ethnography. Unpublished Ph.D. thesis, University of London.

Wendt, W.E., 1974. 'Art mobilier' aus der Apollo 11-Grotte in Sudwest-Africa: die altesten datierten Kunstwerke Afrikas. Acta Praehistorica et Archaeologica 5, 1-42.

White, R., 1989. Production and complexity and standardization in early Aurignacian bead and pendant manufacture: evolutionary implications, in Human Revolution, eds. P. Mellars \& C. Stringer. Edinburgh: Edinburgh University Press, 366-90.

White, R., 1992. Beyond art: toward an understanding of the origins of material representations in Europe. Annual Review in Anthropology 21, 537-64.

White, R., 1993. Technological and social dimensions of 'Aurignacian-age' body ornaments across Europe, in Before Lascaux: the Complex Record of the Early Upper Palaeolithic, eds. H. Knecht, A. Pike-Tay \& R. White. New York (NY): CRC Press, 277-99.

Whiten, A., 1996. When does smart behaviour become mind-reading?, in Theories of Theories of Mind, eds. P. Carruthers \& P.K. Smith. Cambridge: Cambridge University Press, 277-92.

Wolpoff, M. \& R. Caspari, 1996. Why aren't Neandertals modern humans, in The Lower and Middle Palaeolithic, eds. O. Bar-Yosef, L. Cavalli-Sforza, R.J. March \& M. Piperno. (Colloquium IX and X, XIII UISPP Congress.) Forlì: A.B.A.C.O., 133-56.

Wreschner, E., 1982. Red ochre, the transition between the Lower and the Middle Palaeolithic and the origin of modern man, in The Transition from Lower to Middle Palaolithic and the Origin of Modern Man, ed. A. Ronen. (British Archaeological Reports 151.) Oxford: BAR, 35-9.

Wynn, T., 1993. Layers of thinking in tool behavior, in Gibson \& Ingold (eds.), 389-406.

Zilhão, J. \& F. d'Errico, 1999. The chronology and taphonomy of the earliest Aurignacien and its implications for the undestranding of neanderthal extinction. Journal of World Prehistory 13(1), 1-68.

Zilhão, J. \& F. d'Errico, in press. Reply to Straus, Mellars and Otte. Current Anthropology. 